Supporting Information

For

\title{
Silver-catalyzed Annulation of Arynes with Nitriles for Synthesis of Structurally Diverse Quinazolines
}

\author{
Sourav Ghorai ${ }^{\dagger}$, Yongjia Lin ${ }^{\ddagger}$, Yuanzhi Xia ${ }^{\ddagger *}$, Donald J. Wink ${ }^{\dagger}$, and Daesung Lee ${ }^{\dagger *}$ \\ ${ }^{+}$Department of Chemistry, University of Illinois at Chicago \\ ${ }^{\ddagger}$ College of Chemistry and Materials Engineering, Wenzhou University \\ E-mail: dsunglee@uic.edu (D. L.) \\ xiayz04@mails.ucas.ac.cn (Y.X.)
}

\section{Contents}

General Information

S1

Experimental Details and General Procedures

S2-S6

Characterization Data of Substrates

S7-S8

Characterization Data of Products

S9-S16

${ }^{1} \mathrm{H}$ and ${ }^{13} \mathrm{C}$ NMR Spectra of Substrates

S17-S29

${ }^{1} \mathrm{H}$ and ${ }^{13} \mathrm{C}$ NMR Spectra of Quinazoline Products

S30-S65

X-Ray Structure of $\mathbf{2} \mathbf{c a}$ and $\mathbf{2 k a}$

S66-S67

Computational Details

$\mathrm{S} 68-\mathrm{S} 80$ 


\section{General Information}

All reactions were carried out in oven or flame-dried glassware unless otherwise noted. Reagents which were commercially available, were purchased from Sigma - Aldrich, Alfa Aesar, Acros, and Oakwood Products unless otherwise noted. Known compounds were prepared according to literature procedure. Anhydrous acetonitrile from Sigma-Aldrich was distilled over calcium hydride $\left(\mathrm{CaH}_{2}\right)$ under nitrogen atmosphere. Column chromatography was performed using silica gel $60 \AA$ (32-63 mesh) purchased from Silicycle Inc. Analytical thin layer chromatography (TLC) was performed on $0.25 \mathrm{~mm}$ E. Merck precoated silica gel 60 (particle size $0.040-0.063 \mathrm{~mm}$ ). Yield was calculated on basis of chromatographically and spectroscopically pure isolated compound. ${ }^{1} \mathrm{H}$ NMR and ${ }^{13} \mathrm{C}$ NMR spectra were recorded on a Bruker AV-500 spectrometer. ${ }^{1} \mathrm{H}$ NMR chemical shifts $(\delta)$ were reported in parts per million (ppm) downfield of TMS and were referenced relative to the residual proteated solvent peak $\left(\mathrm{CDCl}_{3}(7.26 \mathrm{ppm})\right) \cdot{ }^{13} \mathrm{C}$ chemical shifts $(\delta)$ were reported in parts per million downfield of TMS and are referenced to the carbon resonance of the solvent $\left(\mathrm{CDCl}_{3}, \delta 77.2\right.$ ppm). Multiplicities in ${ }^{1} \mathrm{H}$ NMR were abbreviated by $\mathrm{s}$ (singlet), $\mathrm{d}$ (doublet), $\mathrm{t}$ (triplet), $\mathrm{q}$ (quartet), quin (quintet), sext (sextet), sept (septet) or $\mathrm{m}$ (multiplet). ${ }^{1} \mathrm{H}$ NMR signals that fall within a ca. $0.3 \mathrm{ppm}$ range are generally reported as a multiplet, with a range of chemical shift values corresponding to the peak or centre of the peak. Coupling constants, $J$, are reported in $\mathrm{Hz}$ (Hertz). Electrospray ionization (ESI) mass spectra were recorded on a Waters Micromass Q-Tof Ultima (Waters Corporation, Milford, MA, USA) at the University of Illinois at Urbana-Champaign. Electron impact (EI) mass spectra was obtained using a Micromass 70-VSE (Waters Corporation, Milford, MA, USA) at the University of Illinois at UrbanaChampaign. 


\section{Experimental Details}

General Procedure for Synthesis of Symmetrical bis-1,3-Diyne (1a-d, 1f-i)

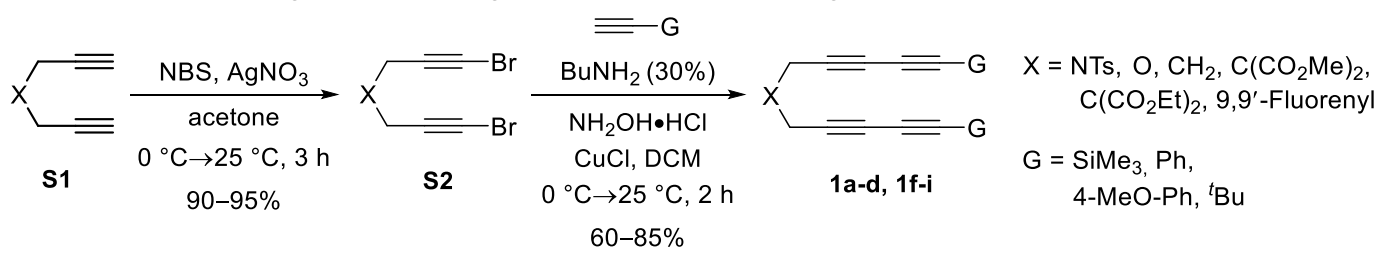

To a stirred solution of diyne $(10.0 \mathrm{mmol})$ in acetone at $0{ }^{\circ} \mathrm{C}, \mathrm{N}$-bromosuccinimide $(25.0 \mathrm{mmol})$ and $\mathrm{AgNO}_{3}(1.0 \mathrm{mmol})$ were added sequentially under $\mathrm{N}_{2}$ atmosphere in the dark. ${ }^{1}$ After addition, the ice bath was removed and stirring was continued for additional $3 \mathrm{~h}$. Upon complete consumption of alkyne, the reaction mixture was concentrated under reduced pressure and filtered through silica gel. Purification by flash column chromatography (SiO, EtOAc-hexanes, 1:5 to 1:3) provided dibromide (S2) in excellent yield.

To a mixture of $30 \%$ aq. $n-\mathrm{BuNH}_{2}(10 \mathrm{~mL}, 2 \mathrm{~mL}$ per $1 \mathrm{mmol}$ of terminal alkyne) and $\mathrm{CuCl}$ (1.5 $\mathrm{mmol})$ in a two-necked round-bottom flask, a solution of terminal alkyne $(5.0 \mathrm{mmol})$ in $\mathrm{CH}_{2} \mathrm{Cl}_{2}$ was added slowly at $0{ }^{\circ} \mathrm{C}$ under $\mathrm{N}_{2}$ atmosphere. ${ }^{2}$ Then $\mathbf{S 2}(2.0 \mathrm{mmol})$ diluted in $\mathrm{CH}_{2} \mathrm{Cl}_{2}$ was added dropwise into the reaction mixture over $45 \mathrm{~min}$. After $5 \mathrm{~min}$, the ice bath was removed and stirring was continued for additional $1-2 \mathrm{~h}$ at room temperature (TLC monitoring). A pinch of $\mathrm{NH}_{2} \mathrm{OH} \cdot \mathrm{HCl}$ was added several times into the reaction mixture when the solution becomes blue. The reaction was quenched by a sat. solution of $\mathrm{NH}_{4} \mathrm{Cl}$ and extracted with $\mathrm{CH}_{2} \mathrm{Cl}_{2}$. The combined organic extracts were washed with water and brine sequentially and dried over anhydrous $\mathrm{Na}_{2} \mathrm{SO}_{4}$ and concentrated under reduced pressure. The crude material was purified by flash column chromatography (SiO, EtOAc-hexanes, 1:20 to 1:10) to isolate pure symmetrical bis-1,3-diyne in moderate to good yield.

\section{Procedure for Synthesis of Unsymmetrical bis-1,3-Diyne (1j)}

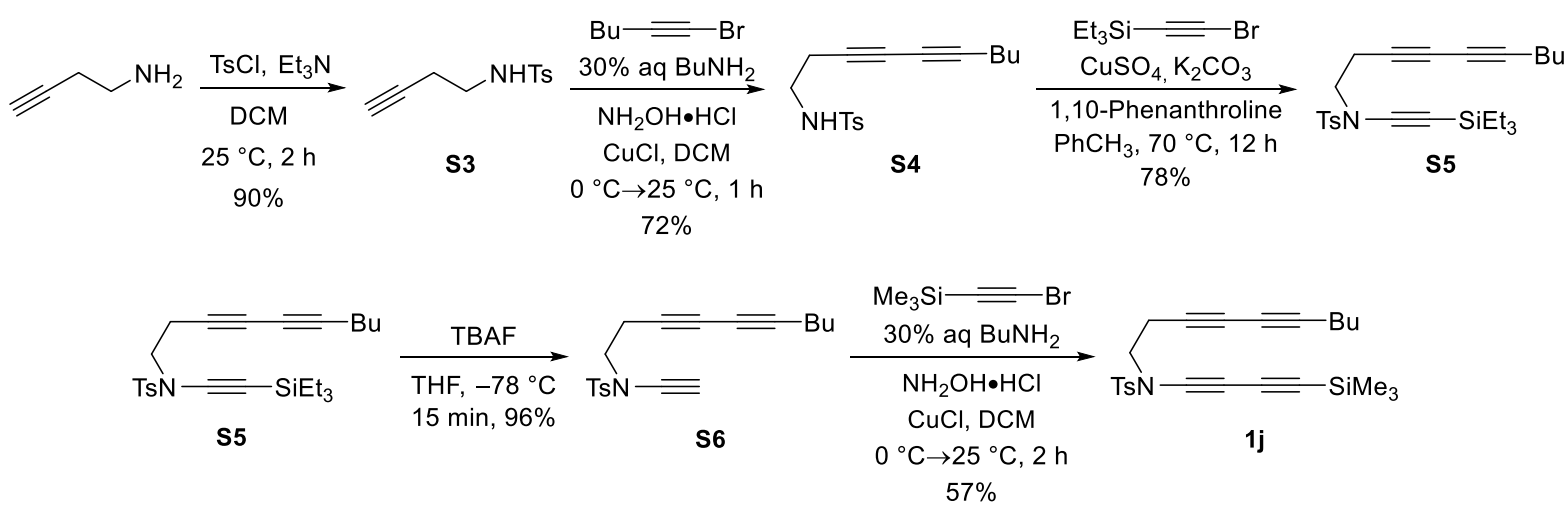

To a stirred solution of 3-butynylamine $(1.5 \mathrm{~g}, 21.7 \mathrm{mmol})$ in $\mathrm{CH}_{2} \mathrm{Cl}_{2}$ at $25^{\circ} \mathrm{C}, \mathrm{Et}_{3} \mathrm{~N}(4.4 \mathrm{~g}, 43.4$ $\mathrm{mmol}$ ) and 4-toluenesulfonyl chloride $(4.1 \mathrm{~g}, 21.7 \mathrm{mmol})$ were added sequentially under $\mathrm{N}_{2}$. After 2 $h$, the reaction mixture was quenched with sat. $\mathrm{NaHCO}_{3}$ and extracted with $\mathrm{CH}_{2} \mathrm{Cl}_{2}(\mathrm{x} 2)$. The combined organic extracts were washed with water and brine and dried over anhydrous $\mathrm{Na}_{2} \mathrm{SO}_{4}$ and concentrated under reduced pressure. Crude S3 (4.3 g, 90\%) was subjected to the next reaction without further purification.

To a mixture of $30 \%$ aq. $n-\mathrm{BuNH}_{2}(9 \mathrm{~mL})$ and $\mathrm{CuCl}(133 \mathrm{mg}, 1.3 \mathrm{mmol})$ in a two-necked round-bottom flask, a solution of $\mathbf{S 3}(1.0 \mathrm{~g}, 4.5 \mathrm{mmol})$ in $\mathrm{CH}_{2} \mathrm{Cl}_{2}$ was added slowly at $0{ }^{\circ} \mathrm{C}$ under $\mathrm{N}_{2}$ atmosphere. ${ }^{3}$ Then 1-bromohex-1-yne, $\left(598 \mathrm{mg}, 3.7 \mathrm{mmol}\right.$ ) diluted in $\mathrm{CH}_{2} \mathrm{Cl}_{2}$ was added dropwise into the reaction mixture over $30 \mathrm{~min}$. After $5 \mathrm{~min}$, the ice bath was removed and stirring was 
continued for additional $1 \mathrm{~h}$ at room temperature. A pinch of $\mathrm{NH}_{2} \mathrm{OH} \cdot \mathrm{HCl}$ was added several times into reaction mixture when the solution becomes blue. The reaction was quenched by a sat. solution of $\mathrm{NH}_{4} \mathrm{Cl}$ and extracted with $\mathrm{CH}_{2} \mathrm{Cl}_{2}$. The combined organic extracts were washed with water and brine sequentially and dried over anhydrous $\mathrm{Na}_{2} \mathrm{SO}_{4}$ and concentrated under reduced pressure. The crude material was purified by flash column chromatography (SiO, EtOAc-hexanes, 1:10) to isolate pure diyne tosylamide $\mathbf{S 4}$ (980 $\mathrm{mg}, 72 \%)$.

To a stirred solution of $\mathbf{S} 4(500 \mathrm{mg}, 1.6 \mathrm{mmol})$ in $\mathrm{PhCH}_{3}, \mathrm{CuSO}_{4}(62 \mathrm{mg}, 0.2 \mathrm{mmol}), 1,10-$ phenanthroline $(88 \mathrm{mg}, 0.5 \mathrm{mmol})$, and $\mathrm{K}_{2} \mathrm{CO}_{3}(458 \mathrm{mg}, 3.3 \mathrm{mmol})$ were sequentially added under $\mathrm{N}_{2}$ atmosphere at room temperature. ${ }^{3}$ After dropwise addition of (Bromoethynyl)triethylsilane $(440 \mathrm{mg}$, $2.5 \mathrm{mmol}$ ) in $\mathrm{PhCH}_{3}$, the reaction mixture was warmed up to $70^{\circ} \mathrm{C}$. After $12 \mathrm{~h}$, the reaction mixture was filtered through silica and concentrated under reduced pressure. The crude material was purified by flash column chromatography (SiO, EtOAc-hexanes, 1:10) to isolate pure triyne S5 (513 $\mathrm{mg}, 78 \%)$.

To a stirred solution of $\mathbf{S 5}(513 \mathrm{mg}, 1.3 \mathrm{mmol})$ in dry THF was added TBAF (1.4 mL, $1 \mathrm{M}$ solution in THF, $1.4 \mathrm{mmol}$ ) slowly at $-78{ }^{\circ} \mathrm{C}$ under $\mathrm{N}_{2}$ atmosphere. ${ }^{4}$ After $15 \mathrm{~min}$, the reaction mixture was quenched with sat. $\mathrm{NH}_{4} \mathrm{Cl}$ and extracted with EtOAc (x2). The combined organic extracts were washed with water and brine sequentially and dried over anhydrous $\mathrm{Na}_{2} \mathrm{SO}_{4}$ and concentrated under reduced pressure. Crude $\mathbf{S 6}$ (400 $\mathrm{mg}$, 96\%) was subjected to the next reaction without further purification.

To a mixture of $30 \%$ aq. $n$-BuNH $2(2.5 \mathrm{~mL})$ and $\mathrm{CuCl}(36 \mathrm{mg}, 0.4 \mathrm{mmol})$ in a two-necked round-bottom flask, a solution of $\mathbf{S 6}(400 \mathrm{mg}, 1.2 \mathrm{mmol})$ in $\mathrm{CH}_{2} \mathrm{Cl}_{2}$ was added slowly at $0{ }^{\circ} \mathrm{C}$ under $\mathrm{N}_{2}$ atmosphere. Then (bromoethynyl)trimethylsilane, $\left(324 \mathrm{mg}, 1.8 \mathrm{mmol}\right.$ ) diluted in $\mathrm{CH}_{2} \mathrm{Cl}_{2}$ was added dropwise into the reaction mixture over $30 \mathrm{~min}$. After $5 \mathrm{~min}$, the ice bath was removed and stirring was continued for additional $1 \mathrm{~h}$ at room temperature. A pinch of $\mathrm{NH}_{2} \mathrm{OH} \cdot \mathrm{HCl}$ was added several times into reaction mixture when the solution becomes blue. The reaction was quenched by a sat. solution of $\mathrm{NH}_{4} \mathrm{Cl}$ and extracted with $\mathrm{CH}_{2} \mathrm{Cl}_{2}$. The combined organic extracts were washed with water and brine sequentially and dried over anhydrous $\mathrm{Na}_{2} \mathrm{SO}_{4}$ and concentrated under reduced pressure. The crude material was purified by flash column chromatography (SiO, EtOAc-hexanes, $1: 15)$ to isolate pure tetryne $\mathbf{1 j}$ ( $294 \mathrm{mg}, 72 \%)$.

\section{Procedure for Synthesis of a Triyne with an Ester Linker (1k)}

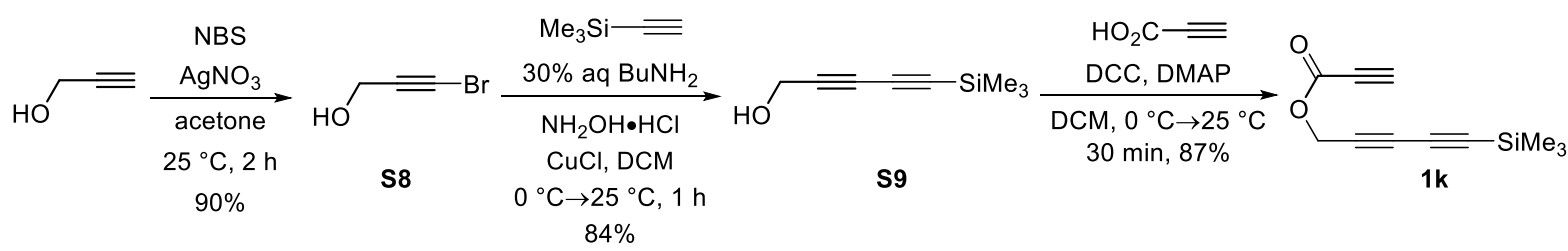

To a stirred solution of 2-propyn-1-ol $(5.0 \mathrm{~g}, 89.1 \mathrm{mmol})$ in acetone at $0{ }^{\circ} \mathrm{C}, \mathrm{N}$ bromosuccinimide $(19.8 \mathrm{~g}, 111.5 \mathrm{mmol})$ and $\mathrm{AgNO}_{3}(1.5 \mathrm{~g}, 8.9 \mathrm{mmol})$ were added sequentially under $\mathrm{N}_{2}$ atmosphere in the dark. After addition, the ice bath was removed and stirring was continued for additional $2 \mathrm{~h}$ at room temperature. Upon complete consumption of alkyne, the reaction mixture was concentrated under reduced pressure and filtered through silica gel. Purification by flash column chromatography (SiO, EtOAc-hexanes, 1:5) provided pure bromide $\mathbf{S 8}$ in excellent yield (7.9 g, 90\%).

To a mixture of $30 \%$ aq. $n$-BuNH $2(22 \mathrm{~mL})$ and $\mathrm{CuCl}(329 \mathrm{mg}, 3.3 \mathrm{mmol})$ in a two-necked round-bottom flask, a solution of ethynyltrimethylsilane $(1.1 \mathrm{~g}, 11.1 \mathrm{mmol})$ in $\mathrm{CH}_{2} \mathrm{Cl}_{2}$ was added slowly at $0{ }^{\circ} \mathrm{C}$ under $\mathrm{N}_{2}$ atmosphere. Then bromide $\mathbf{S 8}(1.0 \mathrm{~g}, 7.4 \mathrm{mmol})$ diluted in $\mathrm{CH}_{2} \mathrm{Cl}_{2}$ was added dropwise over $30 \mathrm{~min}$. After $5 \mathrm{~min}$, the ice bath was removed and stirring was continued for 
additional $1 \mathrm{~h}$. A pinch of $\mathrm{NH}_{2} \mathrm{OH} \cdot \mathrm{HCl}$ was added several times into the reaction mixture when the solution becomes blue. The reaction was quenched by a sat. solution $\mathrm{NH}_{4} \mathrm{Cl}$ and extracted with $\mathrm{CH}_{2} \mathrm{Cl}_{2}$. The combined organic extracts were washed with water and brine sequentially and dried over anhydrous $\mathrm{Na}_{2} \mathrm{SO}_{4}$ and concentrated under reduced pressure. The crude material was purified by flash column chromatography (SiO, EtOAc-hexanes, 1:10) to isolate pure 2,4-diyne-1ol S9 in excellent yield (1.0 g, 84\%).

To a solution of $\mathbf{S 9}\left(500 \mathrm{mg}, 3.3 \mathrm{mmol}\right.$ ) and propiolic acid $(276 \mathrm{mg}, 4.0 \mathrm{mmol})$ in $\mathrm{CH}_{2} \mathrm{Cl}_{2}$ at 0 ${ }^{\circ} \mathrm{C}$, DCC (814 mg, $\left.4.0 \mathrm{mmol}\right)$ and DMAP $(40 \mathrm{mg}, 0.3 \mathrm{mmol})$ were added sequentially under $\mathrm{N}_{2}$ atmosphere. ${ }^{5}$ After addition, the ice bath was removed and stirring was continued at room temperature for additional $30 \mathrm{~min}$. Then, the reaction mixture was concentrated under vacuum and the crude material was purified by flash column chromatography (SiO, EtOAc-hexanes, 1:15) to isolate pure triyne $\mathbf{1 k}$ (584 $\mathrm{mg}, 87 \%$ ).

\section{Procedure for Synthesis of a Triyne with a Phenylamide Linker (1l)}

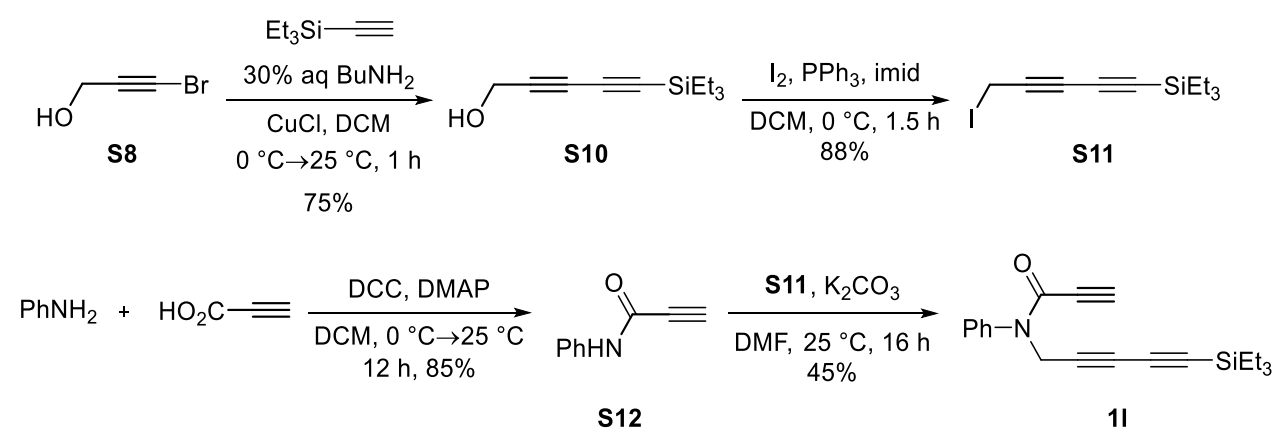

To a mixture of $30 \%$ aq. $n-\mathrm{BuNH}_{2}(22.2 \mathrm{~mL})$ and $\mathrm{CuCl}(331 \mathrm{mg}, 3.4 \mathrm{mmol})$ in a two-necked round-bottom flask, a solution of triethyl(ethynyl)silane $\left(1.6 \mathrm{~g}, 11.2 \mathrm{mmol}\right.$ ) in $\mathrm{CH}_{2} \mathrm{Cl}_{2}$ was added slowly at $0{ }^{\circ} \mathrm{C}$ under $\mathrm{N}_{2}$ atmosphere. Then $\mathbf{S 8}(1.0 \mathrm{~g}, 7.4 \mathrm{mmol})$ diluted in $\mathrm{CH}_{2} \mathrm{Cl}_{2}$ was added dropwise into the reaction mixture over $30 \mathrm{~min}$. After $5 \mathrm{~min}$, the ice bath was removed and stirring was continued for additional $1 \mathrm{~h}$. A pinch of $\mathrm{NH}_{2} \mathrm{OH} \cdot \mathrm{HCl}$ was added several times into the reaction mixture when the solution becomes blue. The reaction was quenched by a sat. solution $\mathrm{NH}_{4} \mathrm{Cl}$ and extracted with $\mathrm{CH}_{2} \mathrm{Cl}_{2}$. The combined organic extracts were washed with water and brine sequentially and dried over anhydrous $\mathrm{Na}_{2} \mathrm{SO}_{4}$ and concentrated under reduced pressure. The crude material was purified by flash column chromatography (SiO, EtOAc-hexanes, 1:10) to isolate pure 2,4-diyne-1ol S10 (1.1 g, 75\%).

To a stirred solution of 2,4-diyne-1ol $\mathbf{S 1 0}(0.8 \mathrm{~g}, 4.1 \mathrm{mmol})$ in $\mathrm{CH}_{2} \mathrm{Cl}_{2}$ at $0{ }^{\circ} \mathrm{C}, \mathrm{PPh}_{3}(1.2 \mathrm{~g}, 4.5$ $\mathrm{mmol}), \mathrm{I}_{2}(1.2 \mathrm{~g}, 4.5 \mathrm{mmol})$, and imidazole $(308 \mathrm{mg}, 4.5 \mathrm{mmol})$ were added sequentially under $\mathrm{N}_{2}$ atmosphere and continued stirring at same temperature. ${ }^{6}$ After $1.5 \mathrm{~h}$, the reaction mixture was quenched by a sat. solution of $\mathrm{Na}_{2} \mathrm{~S}_{2} \mathrm{O}_{3}$ and extracted with $\mathrm{CH}_{2} \mathrm{Cl}_{2}$. The combined organic extracts were washed with water and brine sequentially and dried over anhydrous $\mathrm{Na}_{2} \mathrm{SO}_{4}$ and concentrated under reduced pressure. The crude material was purified by flash column chromatography (SiO, EtOAc-hexanes, 1:20) to isolate pure propagyl iodide $\mathbf{S 1 1}(1.1 \mathrm{~g}, 88 \%)$.

To a stirred solution of aniline $(1.0 \mathrm{~g}, 10.8 \mathrm{mmol})$ and propiolic acid $(0.8 \mathrm{~g}, 11.8 \mathrm{mmol})$ in $\mathrm{Et}_{2} \mathrm{O}$ at $0{ }^{\circ} \mathrm{C}, \mathrm{DCC}(2.7 \mathrm{~g}, 12.9 \mathrm{mmol})$ and DMAP $(131 \mathrm{mg}, 1.1 \mathrm{mmol})$ were added sequentially under $\mathrm{N}_{2}$ atmosphere. After addition, the ice bath was removed and stirring was continued for $12 \mathrm{~h}$ at 25 ${ }^{\circ} \mathrm{C}$. Upon complete consumption of aniline, the reaction mixture was concentrated under reduced pressure and purified by flash column chromatography (SiO, EtOAc-hexanes, 1:3) to isolate pure phenylamide S12 (1.3 g, 85\%). 
To stirred solution of phenylamide $\mathbf{S} 12(286 \mathrm{mg}, 2.0 \mathrm{mmol})$, and propagyl iodide $\mathbf{S} 11$ (500 $\mathrm{mg}, 1.6 \mathrm{mmol})$ in DMF at $25{ }^{\circ} \mathrm{C}, \mathrm{K}_{2} \mathrm{CO}_{3}(452 \mathrm{mg}, 3.3 \mathrm{mmol})$ was added under $\mathrm{N}_{2}$ atmosphere. ${ }^{7}$ After $16 \mathrm{~h}$, the reaction mixture was diluted by water and extracted with EtOAc (x3). The combined organic extracts were washed with water and brine sequentially and dried over anhydrous $\mathrm{Na}_{2} \mathrm{SO}_{4}$ and concentrated under reduced pressure. The crude material was purified by flash column chromatography (SiO, EtOAc-hexanes, 1:3) to isolate pure triyne $\mathbf{1 l}$ in moderate yield (236 mg, 45\%).

\section{Procedure for Synthesis of a Triyne with a Benzoyl Linker (1e)}

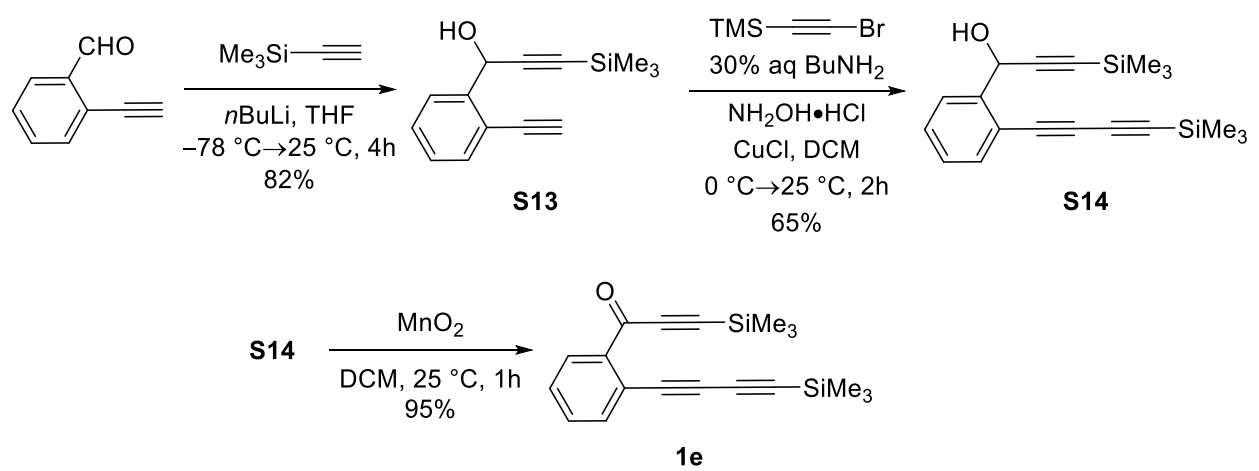

To a stirred solution of ethynyltrimethylsilane $(588 \mathrm{mg}, 6.0 \mathrm{mmol})$ in dry THF was added BuLi (2.2 mL, $2.5 \mathrm{M}$ in hexane, $5.5 \mathrm{mmol}$ ) slowly at $-78{ }^{\circ} \mathrm{C}$ under $\mathrm{N}_{2}$ atmosphere. After $1 \mathrm{~h}, 2-$ ethynylbenzaldehyde $(650 \mathrm{mg}, 5.0 \mathrm{mmol}$ ) diluted in THF was added dropwise at same temperature. After $30 \mathrm{~min}$, the reaction mixture was gradually warmed up to room temperature and the reaction mixture was further stirred for 30 min before it was quenched with sat. $\mathrm{NH}_{4} \mathrm{Cl}$ and extracted with EtOAc (x3). The combined organic extracts were washed with water and brine sequentially and dried over anhydrous $\mathrm{Na}_{2} \mathrm{SO}_{4}$ and concentrated under reduced pressure. The crude material was purified by flash column chromatography (SiO, EtOAc-hexanes, 1:10) to isolate pure diyne S13 $(935 \mathrm{mg}$, $82 \%)$.

To a mixture of $30 \%$ aq. $n-\mathrm{BuNH}_{2}(4.6 \mathrm{~mL})$ and $\mathrm{CuCl}(67 \mathrm{mg}, 0.7 \mathrm{mmol})$ in a two-necked round-bottom flask, a solution of $\mathbf{S} 13(520 \mathrm{mg}, 2.3 \mathrm{mmol})$ in $\mathrm{CH}_{2} \mathrm{Cl}_{2}$ was added slowly at $0{ }^{\circ} \mathrm{C}$ under $\mathrm{N}_{2}$ atmosphere. Then (bromoethynyl)trimethylsilane $\left(605 \mathrm{mg}, 3.4 \mathrm{mmol}\right.$ ) diluted in $\mathrm{CH}_{2} \mathrm{Cl}_{2}$ was added dropwise into the reaction mixture over $30 \mathrm{~min}$. After $5 \mathrm{~min}$, the ice bath was removed and stirring was continued for additional $1 \mathrm{~h}$ at room temperature. A pinch of $\mathrm{NH}_{2} \mathrm{OH} \cdot \mathrm{HCl}$ was added several times into the reaction mixture when the solution becomes blue. The reaction was quenched by a sat. solution of $\mathrm{NH}_{4} \mathrm{Cl}$ and extracted with $\mathrm{CH}_{2} \mathrm{Cl}_{2}$. The combined organic extracts were washed with water and brine sequentially and dried over anhydrous $\mathrm{Na}_{2} \mathrm{SO}_{4}$ and concentrated under reduced pressure. The crude material was purified by flash column chromatography (SiO, EtOAc-hexanes, $1: 15$ ) to isolate pure triyne $\mathbf{S 1 4}$ ( $480 \mathrm{mg}, 65 \%$ ).

To a stirred solution of $\mathbf{S 1 4}\left(480 \mathrm{mg}, 1.5 \mathrm{mmol}\right.$ ) in $\mathrm{CH}_{2} \mathrm{Cl}_{2}, \mathrm{MnO}_{2}$ (645 mg, $7.4 \mathrm{mmol}$ ) was added in one portion under $\mathrm{N}_{2}$ atmosphere at room temperature. After $2 \mathrm{~h}$, the reaction mixture was filtered through celite and concentrated under reduced pressure. Crude 1 e (453 mg, 95\%) was subjected to the next reaction without further purification. 


\section{General Procedure for Generation of Quinazoline}

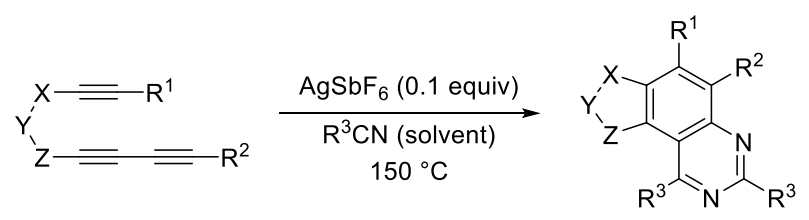

Procedure A: A multiyne substrate $1(0.1 \mathrm{mmol})$ and $\mathrm{AgSbF}_{6}(0.01 \mathrm{mmol})$ in nitrile $(3 \mathrm{~mL})$ in a Schlenk tube was flushed with nitrogen. The mixture was stirred for 0.5 to $5 \mathrm{~h}$ in a heated oil bath $\left(150{ }^{\circ} \mathrm{C}\right)$ unless otherwise noted. The reaction mixture was concentrated, and then directly subjected to flash column chromatography (SiO, EtOAc-hexanes, 1:10 to 1:3) to isolate pure quinazoline product.

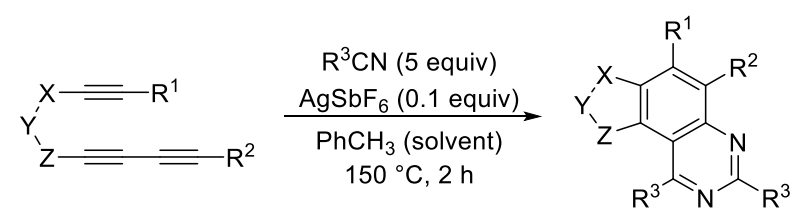

Procedure B: A multiyne substrate $(0.1 \mathrm{mmol})$, nitrile $(0.5 \mathrm{mmol})$ and $\mathrm{AgSbF}_{6}(0.01 \mathrm{mmol})$ in dry $\mathrm{PhCH}_{3}(3 \mathrm{~mL})$ in a Schlenk tube was flushed with nitrogen. The mixture was stirred for 0.5 to $5 \mathrm{~h}$ in a heated oil bath $\left(150{ }^{\circ} \mathrm{C}\right)$ unless otherwise noted. The reaction mixture was concentrated, and then directly subjected to flash column chromatography (SiO, EtOAc-hexanes, 1:10 to 1:3) to isolate pure quinazoline product.

\section{Preparation of Quinazoline 2aa in $1.0 \mathrm{mmol}$ Scale}

Multiyne substrate $1 \mathrm{a}(400 \mathrm{mg}, 1.0 \mathrm{mmol})$ and $\mathrm{AgSbF}_{6}(34.4 \mathrm{mg}, 0.1 \mathrm{mmol})$ in benzonitrile $(10 \mathrm{~mL})$ in a Schlenk tube was flushed with nitrogen. The mixture was stirred for $2 \mathrm{~h}$ in a heated oil bath (150 $\left.{ }^{\circ} \mathrm{C}\right)$. The reaction mixture was concentrated, and then directly subjected to flash column chromatography (SiO, EtOAc-hexanes, $1: 10$ to $1: 3$ ) to isolate pure quinazoline 2 aa $(533 \mathrm{mg}, 88 \%$ yield).

\section{References:}

[1] Hofmeister, H.; Annen, K.; Laurent, H.; Wiechert, H. Angew. Chem., Int. Ed. 1984, 23, 727.

[2] (a) Chodkiewicz, W. Ann. Chim. 1957, 2, 819. (b) Cadiot, P.; Chodkiewicz, W. In Chemistry of Acetylenes (Ed.: H. G. Viehe), Marcel Dekker, New York, 1969, pp. 597-647.

[3] Zhang, Y.; Hsung, R. P.; Tracey, M. R.; Kurtz, K. C. M.; Vera, E. L. Org. Lett. 2004, 6, 1151.

[4] Nakamura, E.; Kuwajima, I. Angew. Chem., Int. Ed. 1976, 15, 498.

[5] Neises, B.; Steglich, W. Org. Synth. 1985, 63, 183.

[6] Garegg, P. J.; Samuelsson, B. J. Chem. Soc., Chem. Commun. 1979, 978.

[7] Karmakar, R.; Yun, S. Y.; Chen, J.; Xia, Y.; Lee. D. Angew. Chem., Int. Ed. 2015, 54, 6582. 


\section{Characterization Data of Substrates}
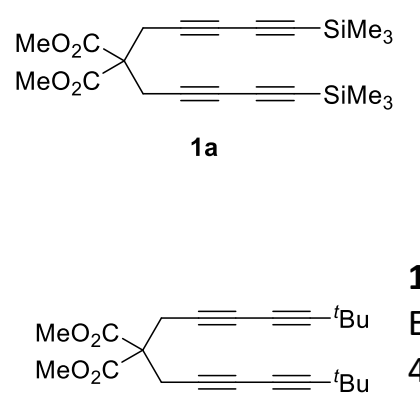

1b

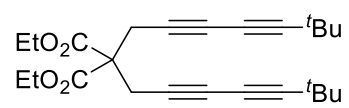

$1 b^{\prime}$

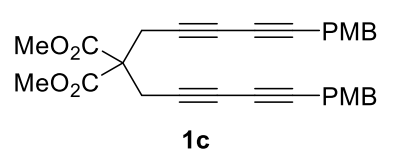

1a: $746 \mathrm{mg}$, white solid, purified by flash column chromatography (SiO, EtOAc-hexanes, 1:20). ${ }^{1} \mathbf{H}$ NMR ( $\left.\mathrm{CDCl}_{3}, 500 \mathrm{MHz}\right): \delta 3.77(\mathrm{~s}, 6 \mathrm{H}), 3.04(\mathrm{~s}$, 4H), $0.18(\mathrm{~s}, 18 \mathrm{H}) ;{ }^{13} \mathrm{C}$ NMR $\left(\mathrm{CDCl}_{3}, 125 \mathrm{MHz}\right): \delta$ 168.4, 87.7, 84.8, 72.8, $68.8,56.4,53.3,23.8,-0.5$; HRMS (ESI) calcd for $\mathrm{C}_{21} \mathrm{H}_{29} \mathrm{O}_{4} \mathrm{Si}_{2}[\mathrm{M}+\mathrm{H}]^{+}$ 401.1604, found 401.1612 .

1b: $436 \mathrm{mg}$, white solid, purified by flash column chromatography (SiO, EtOAc-hexanes, 1:20). ${ }^{1} \mathbf{H}$ NMR $\left(\mathrm{CDCl}_{3}, 500 \mathrm{MHz}\right): \delta 3.75(\mathrm{~s}, 6 \mathrm{H}), 3.05(\mathrm{~s}$, $4 \mathrm{H}), 1.21(\mathrm{~s}, 18 \mathrm{H}) ;{ }^{13} \mathrm{C}$ NMR $\left(\mathrm{CDCl}_{3}, 125 \mathrm{MHz}\right): \delta$ 168.8; 86.3; 71.6; 68.5; 63.6; 56.6; 53.3; 30.5; 27.9, 23.8; HRMS (ESI) calcd for $\mathrm{C}_{23} \mathrm{H}_{29} \mathrm{O}_{4}[\mathrm{M}+\mathrm{H}]^{+}$ 369.2066 , found 369.2071 .

1b': $281 \mathrm{mg}$, white solid, purified by flash column chromatography (SiO, EtOAc-hexanes, 1:20). ${ }^{1} \mathrm{H}$ NMR $\left(\mathrm{CDCl}_{3}, 500 \mathrm{MHz}\right): \delta 4.22(\mathrm{q}, 4 \mathrm{H}, J=7.1 \mathrm{~Hz})$, $3.04(\mathrm{~s}, 4 \mathrm{H}), 1.25(\mathrm{t}, 6 \mathrm{H}, J=7.1 \mathrm{~Hz}), 1.20(\mathrm{~s}, 9 \mathrm{H}) ;{ }^{13} \mathrm{C}$ NMR $\left(\mathrm{CDCl}_{3}, 125 \mathrm{MHz}\right): \delta$ $168.4,86.2,71.8,68.5,63.6,62.2,56.6,30.5,27.9,23.7,14.0 ;$ HRMS (ESI) calcd for $\mathrm{C}_{25} \mathrm{H}_{33} \mathrm{O}_{4}[\mathrm{M}+\mathrm{H}]^{+} 397.2379$, found 397.2377 .

1c: $315 \mathrm{mg}$, yellow solid, purified by flash column chromatography (SiO, EtOAc-hexanes, 1:10). ${ }^{1} \mathbf{H}$ NMR $\left(\mathrm{CDCl}_{3}, 500 \mathrm{MHz}\right): \delta 7.38(\mathrm{~d}, 4 \mathrm{H}, J=8.5$ $\mathrm{Hz}), 6.80(\mathrm{~d}, 4 \mathrm{H}, J=8.5 \mathrm{~Hz}), 3.78(\mathrm{~s}, 6 \mathrm{H}), 3.77(\mathrm{~s}, 6 \mathrm{H}), 3.19(\mathrm{~s}, 4 \mathrm{H}) ;{ }^{13} \mathrm{C} \mathrm{NMR}$ $\left(\mathrm{CDCl}_{3}, 125 \mathrm{MHz}\right): \delta 168.7,160.3,134.2,114.1,113.4,77.0,76.2,72.6$, 68.7, 56.7, 55.3, 53.4, 24.2; HRMS (ESI) calcd for $\mathrm{C}_{29} \mathrm{H}_{25} \mathrm{O}_{6}[\mathrm{M}+\mathrm{H}]^{+} 469.1651$, found 469.1666.

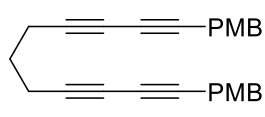

1d

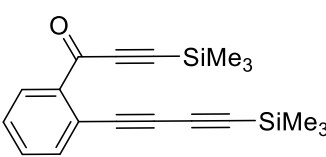

$1 \mathrm{e}$

1d: $276 \mathrm{mg}$, white solid, purified by flash column chromatography (SiO, EtOAchexanes, 1:20). ${ }^{1} \mathrm{H}$ NMR $\left(\mathrm{CDCl}_{3}, 500 \mathrm{MHz}\right): \delta 7.41(\mathrm{~d}, 4 \mathrm{H}, J=8.6 \mathrm{~Hz}), 6.82(\mathrm{~d}, 4 \mathrm{H}$, $J=8.6 \mathrm{~Hz}$ ), $3.80(\mathrm{~s}, 6 \mathrm{H}), 2.52\left(\mathrm{t}, 4 \mathrm{H}, J=6.9 \mathrm{z}\right.$ ), 1.83 (pent, $2 \mathrm{H}, J=6.9 \mathrm{z}$ ); ${ }^{13} \mathrm{C}$ NMR $\left(\mathrm{CDCl}_{3}, 125 \mathrm{MHz}\right): \delta 160.1,134.1,114.1,113.8,82.5,75.3,73.0,66.2,55.3,27.1$, 18.8; HRMS (ESI) calcd for $\mathrm{C}_{25} \mathrm{H}_{21} \mathrm{O}_{2}[\mathrm{M}+\mathrm{H}]^{+} 353.1542$, found 353.1556 .

1e: $453 \mathrm{mg}$, colourless oil, purified by flash column chromatography (SiO, EtOAc-hexanes, 1:20). ${ }^{1} \mathrm{H}$ NMR $\left(\mathrm{CDCl}_{3}, 500 \mathrm{MHz}\right): \delta 8.06(\mathrm{~d}, 1 \mathrm{H}, J=7.6 \mathrm{~Hz})$, $7.60(\mathrm{~d}, 1 \mathrm{H}, J=7.5 \mathrm{~Hz}), 7.50(\mathrm{t}, 1 \mathrm{H}, J=7.3 \mathrm{~Hz}), 7.46(\mathrm{~d}, 1 \mathrm{H}, J=7.5 \mathrm{~Hz}), 0.30$ (s, 9H), 0.22 (s, 9H); ${ }^{13} \mathrm{C}$ NMR (CDCl $\left.3,125 \mathrm{MHz}\right): \delta$ 176.4, 139.2, 135.8, 132.6, 131.6, 128.9, 121.4, 101.8, 101.4, 93.0, 88.1, 80.3, 74.5, -0.4, -0.7; HRMS (ESI) calcd for $\mathrm{C}_{19} \mathrm{H}_{23} \mathrm{OSi}_{2}[\mathrm{M}+\mathrm{H}]^{+} 323.1287$, found 323.1288 .
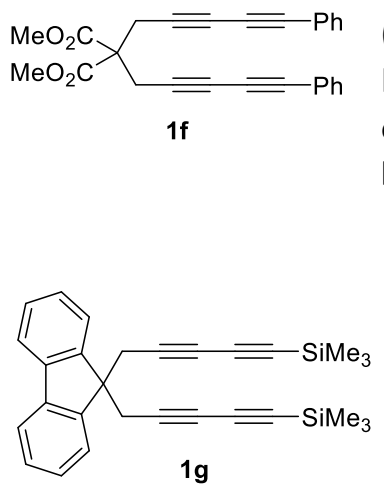

1f: $360 \mathrm{mg}$, orange-yellow solid, purified by flash column chromatography (SiO, EtOAc-hexanes, 1:10). ${ }^{1} \mathrm{H}$ NMR $\left(\mathrm{CDCl}_{3}, 500 \mathrm{MHz}\right): \delta 7.48$ (d, $4 \mathrm{H}, J=6.9$ $\mathrm{Hz}), 7.38-7.27(\mathrm{~m}, 6 \mathrm{H}), 3.81(\mathrm{~s}, 6 \mathrm{H}), 3.22(\mathrm{~s}, 4 \mathrm{H}) ;{ }^{13} \mathrm{C} \mathrm{NMR}\left(\mathrm{CDCl}_{3}, 125 \mathrm{MHz}\right)$ : $\delta$ 168.7, 132.6, 129.2, 128.4, 121.5, 77.7, 76.1, 73.8, 68.5, 56.7, 53.4, 24.3;

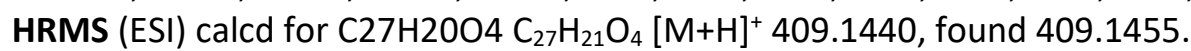

1g: $378 \mathrm{mg}$, white solid, purified by flash column chromatography (SiO, EtOAc-hexanes, 1:20). ${ }^{1} \mathbf{H}$ NMR $\left(\mathrm{CDCl}_{3}, 500 \mathrm{MHz}\right): \delta 7.77-7.67(\mathrm{~m}, 4 \mathrm{H})$, $7.40(\mathrm{t}, 2 \mathrm{H}, J=7.2 \mathrm{~Hz}), 7.35(\mathrm{t}, 2 \mathrm{H}, J=7.2 \mathrm{~Hz}), 2.89(\mathrm{~s}, 4 \mathrm{H}), 0.21(\mathrm{~s}, 18 \mathrm{H})$; ${ }^{13} \mathrm{C}$ NMR $\left(\mathrm{CDCl}_{3}, 125 \mathrm{MHz}\right): \delta 147.8,139.7,128.3,127.6,123.9,120.1$, 
88.2, 84.2, 76.1, 68.0, 50.4, 28.4, -0.3; HRMS (EI) calcd for $\mathrm{C}_{29} \mathrm{H}_{30} \mathrm{Si}_{2}$ [M] 434.18861, found 434.18865.

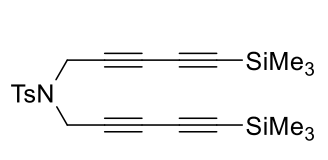

$1 \mathrm{~h}$

440.1531.

1h: $460 \mathrm{mg}$, white solid, purified by flash column chromatography (SiO, EtOAc-hexanes, 1:20). ${ }^{1} \mathrm{H}$ NMR $\left(\mathrm{CDCl}_{3}, 500 \mathrm{MHz}\right): \delta 7.68(\mathrm{~d}, 2 \mathrm{H}, J=8.2 \mathrm{~Hz})$, $7.32(\mathrm{~d}, 2 \mathrm{H}, J=8.2 \mathrm{~Hz}), 4.18(\mathrm{~s}, 4 \mathrm{H}), 2.42(\mathrm{~s}, 3 \mathrm{H}), 0.18(\mathrm{~s}, 18 \mathrm{H}) ;{ }^{13} \mathrm{C}$ NMR $\left(\mathrm{CDCl}_{3}, 125 \mathrm{MHz}\right): \delta$ 144.3, 134.5, 129.8, 127.9, 87.0, 86.9, 71.0, 70.1, 37.3, 21.6, -0.5 ; HRMS (ESI) calcd for $\mathrm{C}_{23} \mathrm{H}_{30} \mathrm{NO}_{2} \mathrm{SSi}_{2}[\mathrm{M}+\mathrm{H}]^{+} 440.1536$, found

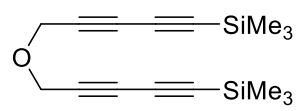

$1 \mathrm{i}$

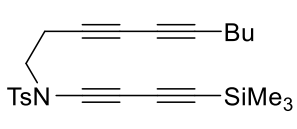

1j

1i: $242 \mathrm{mg}$, colourless oil, purified by flash column chromatography (SiO, EtOAc-hexanes, 1:20). ${ }^{1} \mathrm{H}$ NMR $\left(\mathrm{CDCl}_{3}, 500 \mathrm{MHz}\right): \delta 4.32(\mathrm{~s}, 4 \mathrm{H}), 0.20(\mathrm{~s}, 18 \mathrm{H})$; ${ }^{13} \mathrm{C}$ NMR $\left(\mathrm{CDCl}_{3}, 125 \mathrm{MHz}\right): \delta 87.7,87.0,72.8,72.0,57.0,-0.5$; HRMS (El) calcd for $\mathrm{C}_{16} \mathrm{H}_{22} \mathrm{OSi}_{2}[\mathrm{M}] 286.12093$, found 286.12162 .

1j: $320 \mathrm{mg}$, orange-yellow solid, purified by flash column chromatography (SiO, EtOAc-hexanes, 1:20). ${ }^{1} \mathrm{H}$ NMR $\left(\mathrm{CDCl}_{3}, 500 \mathrm{MHz}\right): \delta 7.78(\mathrm{~d}, 2 \mathrm{H}, \mathrm{J}=8.3$ $\mathrm{Hz}), 7.36(\mathrm{~d}, 2 \mathrm{H}, J=8.1 \mathrm{~Hz}), 3.49(\mathrm{t}, 2 \mathrm{H}, J=7.6 \mathrm{~Hz}), 2.57(\mathrm{t}, 2 \mathrm{H}, J=7.6 \mathrm{~Hz}), 2.45$ $(\mathrm{s}, 3 \mathrm{H}), 2.24(\mathrm{t}, 2 \mathrm{H}, J=7.0 \mathrm{~Hz}), 1.54-1.45(\mathrm{~m}, 2 \mathrm{H}), 1.45-1.36(\mathrm{~m}, 2 \mathrm{H}), 0.90(\mathrm{t}$, $3 \mathrm{H}, J=7.3 \mathrm{~Hz}), 0.19$ (s, 9H); ${ }^{13} \mathrm{C}$ NMR (CDCl, $\left.125 \mathrm{MHz}\right): \delta$ 145.4, 134.4, 130.1, 127.6, 90.6, 87.3, 78.9, 71.5, 68.4, 67.8, 64.8, 59.6, 49.8, 30.2, 21.9, 21.7, 19.2, 18.9, 13.5, -0.4; HRMS (ESI) calcd for $\mathrm{C}_{24} \mathrm{H}_{30} \mathrm{NO}_{2} \mathrm{SSi}[\mathrm{M}+\mathrm{H}]^{+} 424.1767$, found 424.1775 .

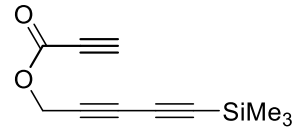

$1 \mathrm{k}$ 204.05987.

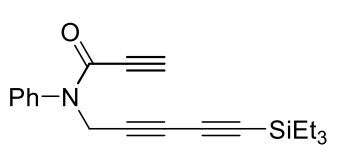

11

1k: $584 \mathrm{mg}$, colourless oil, purified by flash column chromatography (SiO, EtOAc-hexanes, 1:20). ${ }^{1} \mathrm{H}$ NMR $\left(\mathrm{CDCl}_{3}, 500 \mathrm{MHz}\right): \delta 4.78(\mathrm{~s}, 2 \mathrm{H}), 2.98(\mathrm{~s}, 1 \mathrm{H})$, 0.15 (S, 9H); ${ }^{13} \mathrm{C}$ NMR $\left(\mathrm{CDCl}_{3}, 125 \mathrm{MHz}\right): \delta 151.5,88.9,86.7,76.3,73.7,72.3$, 70.0, 53.7, -0.7; HRMS (EI) calcd for $\mathrm{C}_{11} \mathrm{H}_{12} \mathrm{O}_{2} \mathrm{Si}$ [M] 204.06066, found

1l: $235 \mathrm{mg}$, light yellow oil, purified by flash column chromatography (SiO, EtOAc-hexanes, 1:10). ${ }^{1} \mathbf{H}$ NMR $\left(\mathrm{CDCl}_{3}, 500 \mathrm{MHz}\right): \delta$ 7.46-7.37 (m, 3H), 7.36$7.31(\mathrm{~m}, 2 \mathrm{H}), 4.56(\mathrm{~s}, 2 \mathrm{H}), 2.83(\mathrm{~s}, 1 \mathrm{H}), 0.97(\mathrm{t}, 9 \mathrm{H}, J=7.9 \mathrm{~Hz}), 0.60$ (quart, $6 \mathrm{H}$, $J=7.9 \mathrm{~Hz}) ;{ }^{13} \mathrm{C}$ NMR $\left(\mathrm{CDCl}_{3}, 125 \mathrm{MHz}\right): \delta 152.5,140.3,129.5,129.0,128.2$, $88.3,84.7,80.6,75.6,71.4,69.5,38.7,7.3,4.1$; HRMS (ESI) calcd for $\mathrm{C}_{20} \mathrm{H}_{24} \mathrm{NOSi}[\mathrm{M}+\mathrm{H}]^{+} 322.1627$, found 322.1637. 


\section{Characterization Data of Quinazoline Products}

2aa: This product was synthesized using procedure $\mathbf{A}$ and isolated by flash column chromatography (SiO, EtOAc-hexanes, 1:10) as white solid (92\% yield, $58.4 \mathrm{mg}$ ) after stirring the reaction mixture for

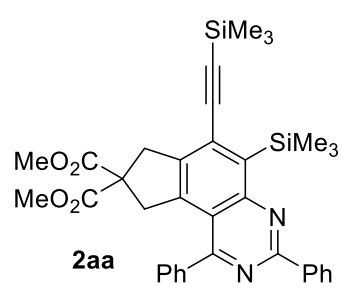

2 h. ${ }^{1} \mathbf{H}$ NMR $\left(\mathrm{CDCl}_{3}, 500 \mathrm{MHz}\right): \delta 8.64-8.60(\mathrm{~m}, 2 \mathrm{H}), 7.60-7.44(\mathrm{~m}, 8 \mathrm{H}), 3.73$ (s, 2H), $3.69(\mathrm{~s}, 6 \mathrm{H}), 3.14(\mathrm{~s}, 2 \mathrm{H}), 0.64(\mathrm{~s}, 9 \mathrm{H}), 0.31(\mathrm{~s}, 9 \mathrm{H}) ;{ }^{13} \mathrm{C}$ NMR $\left(\mathrm{CDCl}_{3}\right.$, $125 \mathrm{MHz}): \delta 171.8,167.9,157.3,156.8,142.3,140.5,138.1,136.4,131.5$, $130.3,129.5,129.0,128.7,128.5,128.3,118.8,105.9,103.8,59.1,53.0$, 43.0, 41.4, 2.9, -0.3; HRMS (ESI) calcd for $\mathrm{C}_{35} \mathrm{H}_{39} \mathrm{~N}_{2} \mathrm{O}_{4} \mathrm{Si}_{2}[\mathrm{M}+\mathrm{H}]^{+} 607.2448$, found 607.2450 .

2ba: This product was synthesized using procedure $\mathbf{A}$ (20 wt\% $4 \AA$ MS was added into reaction

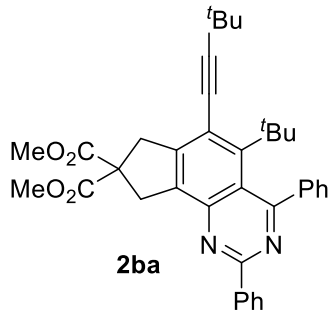
mixture) and isolated by flash column chromatography (SiO, EtOAc-hexanes, 1:10) as orange-yellow solid (69\% yield, $36.7 \mathrm{mg}$ ) after stirring the reaction mixture for $2 \mathrm{~h} .{ }^{1} \mathrm{H}$ NMR $\left(\mathrm{CDCl}_{3}, 500 \mathrm{MHz}\right): 8.65(\mathrm{~d}, 2 \mathrm{H}, J=7.0 \mathrm{~Hz}), 8.48(\mathrm{br}$, $1 \mathrm{H}), 7.76-7.26(\mathrm{~m}, 6 \mathrm{H}), 7.07(\mathrm{br}, 1 \mathrm{H}), 4.16(\mathrm{~s}, 2 \mathrm{H}), 3.97-3.69(\mathrm{~m}, 8 \mathrm{H}), 1.41(\mathrm{~s}$, $9 \mathrm{H}), 1.31(\mathrm{~s}, 9 \mathrm{H}) ;{ }^{13} \mathrm{C} \mathrm{NMR}\left(\mathrm{CDCl}_{3}, 125 \mathrm{MHz}\right): \delta 172.4,165.6,156.5,151.9$, $148.5,147.3,144.5,137.1,133.6,131.0$ (br), 130.5, 130.3, 128.6 (br), 128.5, 120.9, 119.7, 111.1, 78.7, 58.6, 53.1, 42.8, 39.6, 39.5, 31.4, 30.7; HRMS (ESI) calcd for $\mathrm{C}_{37} \mathrm{H}_{39} \mathrm{~N}_{2} \mathrm{O}_{4}[\mathrm{M}+\mathrm{H}]^{+} 575.2910$, found 575.2919 .

2b'a: This product was synthesized using procedure $\mathbf{A}$ (20 wt\% $4 \AA$ MS was used as additive) and isolated by flash column chromatography (SiO, EtOAc-hexanes, $1: 10$ ) as orange yellow solid ( $84 \%$

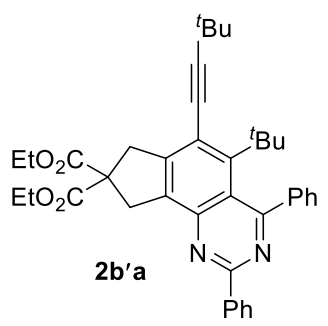
yield, $45.2 \mathrm{mg})$ after stirring the reaction mixture for $2 \mathrm{~h} .{ }^{1} \mathrm{H}$ NMR $\left(\mathrm{CDCl}_{3}\right.$, $500 \mathrm{MHz}): \delta 8.65(\mathrm{~d}, 2 \mathrm{H}, J=7.8 \mathrm{~Hz}), 8.48(\mathrm{br}, 1 \mathrm{H}), 7.69-7.40(\mathrm{~m}, 6 \mathrm{H}), 7.11$ (br, 1H), 4.40-4.04 (m, 6H), $3.87(\mathrm{~d}, 2 \mathrm{H}, J=8.1 \mathrm{~Hz}), 1.41(\mathrm{~s}, 9 \mathrm{H}), 1.37-1.22$ $(\mathrm{m}, 6 \mathrm{H}), 1.31(\mathrm{~s}, 9 \mathrm{H}) ;{ }^{13} \mathrm{C}$ NMR $\left(\mathrm{CDCl}_{3}, 125 \mathrm{MHz}\right): \delta 171.9,165.6,156.4$, $151.7,148.5,147.5,144.5,137.1,133.7,130.4,130.2,129.1,128.5,128.4$, 120.9, 119.7, 111.0, 78.7, 61.8, 58.7, 42.7, 39.5, 31.4, 30.6, 29.7, 28.8, 14.1; HRMS (ESI) calcd for $\mathrm{C}_{39} \mathrm{H}_{43} \mathrm{~N}_{2} \mathrm{O}_{4}[\mathrm{M}+\mathrm{H}]^{+} 603.3223$, found 603.3207 .

2ca: This product was synthesized using procedure $\mathbf{A}$ and isolated by flash column chromatography (SiO, EtOAc-hexanes, 1:5) as orange-yellow solid (66\% yield, $40.3 \mathrm{mg}$ ) after stirring the reaction

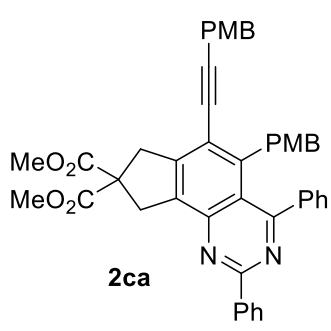
mixture for $2 \mathrm{~h} .{ }^{1} \mathrm{H}$ NMR $\left(\mathrm{CDCl}_{3}, 500 \mathrm{MHz}\right): \delta 8.71(\mathrm{~d}, 2 \mathrm{H}, J=7.8 \mathrm{~Hz}), 7.56-$ $7.46(\mathrm{~m}, 3 \mathrm{H}), 7.30-7.20(\mathrm{~m}, 4 \mathrm{H}), 7.14-7.02(\mathrm{~m}, 5 \mathrm{H}), 6.81(\mathrm{~d}, 2 \mathrm{H}, J=8.7 \mathrm{~Hz})$, $6.56(\mathrm{~d}, 2 \mathrm{H}, J=8.5 \mathrm{~Hz}), 4.34(\mathrm{~s}, 2 \mathrm{H}), 4.06(\mathrm{~s}, 2 \mathrm{H}), 3.86(\mathrm{~s}, 6 \mathrm{H}), 3.80(\mathrm{~s}, 3 \mathrm{H})$, $3.72(\mathrm{~s}, 3 \mathrm{H}) ;{ }^{13} \mathrm{C}$ NMR $\left(\mathrm{CDCl}_{3}, 125 \mathrm{MHz}\right): \delta 172.3,159.9,158.6,149.1,146.4$, $141.7,140.5,137.7,136.3,133.0,132.8,131.5,130.6,130.0,128.7,128.5$, $128.1,127.3,121.0,120.1,115.2,114.0,112.7,97.9,86.3,59.0,55.3,53.2$, 42.7, 39.9; HRMS (ESI) calcd for $\mathrm{C}_{26} \mathrm{H}_{37} \mathrm{~N}_{2} \mathrm{O}_{3} \mathrm{SSi}_{2}[\mathrm{M}+\mathrm{H}]^{+} 513.2063$, found 513.2068 .

$\mathbf{2} \mathbf{c}^{\prime} \mathbf{a}$ : This product was synthesized using procedure $\mathbf{A}$ and isolated by flash column chromatography

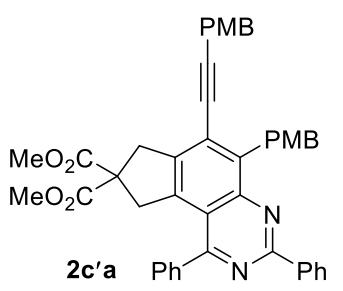

(SiO, EtOAc-hexanes, 1:5) as orange-yellow solid in an inseparable mix with 2ca (88\% overall yield, $53.7 \mathrm{mg}$ ) after stirring the reaction mixture for $2 \mathrm{~h} .{ }^{1} \mathbf{H}$ NMR $\left(\mathrm{CDCl}_{3}, 500 \mathrm{MHz}\right): 8.51-8.43(\mathrm{~m}, 2 \mathrm{H}), 7.74(\mathrm{~d}, 2 \mathrm{H}, \mathrm{J}=8.6 \mathrm{~Hz}), 7.63-7.55$ $(\mathrm{m}, 5 \mathrm{H}), 7.45-7.39(\mathrm{~m}, 3 \mathrm{H}), 7.33(\mathrm{~d}, 2 \mathrm{H}, J=8.7 \mathrm{~Hz}), 7.12(\mathrm{~d}, 2 \mathrm{H}, J=8.6 \mathrm{~Hz})$, $6.86(\mathrm{~d}, 2 \mathrm{H}, J=8.7 \mathrm{~Hz}), 3.97(\mathrm{~s}, 3 \mathrm{H}), 3.90(\mathrm{~s}, 2 \mathrm{H}), 3.83(\mathrm{~s}, 3 \mathrm{H}), 3.72(\mathrm{~s}, 6 \mathrm{H})$, 
$3.20(\mathrm{~s}, 2 \mathrm{H}) ;{ }^{13} \mathrm{C}$ NMR $\left(\mathrm{CDCl}_{3}, 125 \mathrm{MHz}\right): \delta$ (all discernible signals for both isomers) 172.3, 171.8, $168.7,167.7,160.2$, 159.9, 159.1, 158.6, 158.4, 150.5, 149.1, 146.4, 141.7, 141.4, 140.8, 140.5, $138.1,137.7,136.3,134.2,133.3,133.3,133.0,132.8,131.5,130.7,130.3,130.0,129.5,129.3$, $129.1,128.7,128.5,128.4,128.3,128.1,127.3,124.8,121.0,120.1,119.3,115.2,114.9,114.1$, 114.0, 112.7, 112.6, 99.6, 97.9, 86.3, 59.3, 59.0, 55.4, 55.3, 53.2, 53.1, 42.9, 42.7, 41.3, 40.0, 29.7.

2da: This product was synthesized using procedure $\mathbf{A}$ (20 wt\% $4 \AA$ MS was used as additive) and isolated by flash column chromatography (SiO, EtOAc-hexanes, 1:5) as orange-yellow solid $(95 \%$ yield, $52.9 \mathrm{mg})$ after stirring the reaction mixture for $2 \mathrm{~h} .{ }^{1} \mathrm{H}$ NMR $\left(\mathrm{CDCl}_{3}, 500 \mathrm{MHz}\right): \delta 8.73-8.69(\mathrm{~m}$,<smiles>CC(C)=Cc1nc(-c2ccccc2)nc2c1CCC2</smiles>
$2 \mathrm{H}), 7.58-7.45(\mathrm{~m}, 3 \mathrm{H}), 7.31-7.27(\mathrm{~m}, 2 \mathrm{H}), 7.22(\mathrm{~d}, 2 \mathrm{H}, J=8.8 \mathrm{~Hz}), 7.13-7.02(\mathrm{~m}, 5 \mathrm{H})$, $6.80(\mathrm{~d}, 2 \mathrm{H}, J=8.8 \mathrm{~Hz}), 6.56(\mathrm{~d}, 2 \mathrm{H}, J=8.6 \mathrm{~Hz}), 3.80(\mathrm{~s}, 3 \mathrm{H}), 3.72(\mathrm{~S}, 3 \mathrm{H}), 3.65(\mathrm{t}, 2 \mathrm{H}, J$ $=7.5 \mathrm{~Hz}$ ), $3.39(\mathrm{t}, 2 \mathrm{H}, J=7.6 \mathrm{~Hz}), 2.38$ (pent, $2 \mathrm{H}, J=7.5 \mathrm{~Hz}) ;{ }^{13} \mathrm{C}$ NMR $\left(\mathrm{CDCl}_{3}, 125\right.$ $\mathrm{MHz}): \delta 168.5,159.7,158.5,158.4,150.9,149.6,140.8,140.6,140.4,138.0,132.9$, $131.9,130.4,130.0,128.6,128.4,128.0,127.3,120.7,120.6,115.5,114.0,112.7$, 97.2, 87.0, 55.3, 35.1, 31.6, 24.0; HRMS (ESI) calcd for $\mathrm{C}_{39} \mathrm{H}_{31} \mathrm{~N}_{2} \mathrm{O}_{2}[\mathrm{M}+\mathrm{H}]^{+} 559.2386$, found 559.2369 .

2ea: This product was synthesized using procedure $\mathbf{A}$ (20 wt\% $4 \AA$ MS was used as additive) and isolated by flash column chromatography (SiO, EtOAc-hexanes, 1:10) as orange-yellow solid (87\% yield, $45.8 \mathrm{mg})$ after stirring the reaction mixture for $2 \mathrm{~h} .{ }^{1} \mathrm{H} \mathrm{NMR}\left(\mathrm{CDCl}_{3}, 500 \mathrm{MHz}\right): \delta 8.75(\mathrm{~d}, 2 \mathrm{H}, \mathrm{J}=$ $7.7 \mathrm{~Hz}), 7.91(\mathrm{~d}, 2 \mathrm{H}, J=7.5 \mathrm{~Hz}), 7.61-7.52(\mathrm{~m}, 5 \mathrm{H}), 7.51-7.46(\mathrm{~m}, 2 \mathrm{H}), 7.09(\mathrm{t}$,

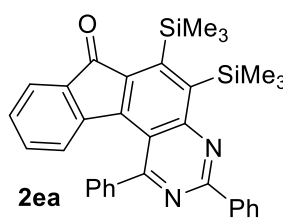
$1 \mathrm{H}, J=7.4 \mathrm{~Hz}), 6.88(\mathrm{t}, 1 \mathrm{H}, J=7.6 \mathrm{~Hz}), 5.98(\mathrm{~d}, 1 \mathrm{H}, J=7.7 \mathrm{~Hz}), 0.61(\mathrm{~s}, 9 \mathrm{H}), 0.54$ (s, 9H); ${ }^{13} \mathrm{C}$ NMR $\left(\mathrm{CDCl}_{3}, 125 \mathrm{MHz}\right): \delta 195.1,167.0,159.0,157.0,155.5,143.5$, $143.1,140.4,137.6,133.3,133.0,130.9,130.6,128.9,128.8,128.7,128.6$, 126.2, 123.1, 115.3, 3.8, 2.8; HRMS (ESI) calcd for $\mathrm{C}_{33} \mathrm{H}_{33} \mathrm{~N}_{2} \mathrm{OSi}{ }_{2}[\mathrm{M}+\mathrm{H}]^{+}$ 529.2131 , found 529.2148 .

2fa: This product was synthesized using procedure $\mathbf{A}$ and isolated by flash column chromatography

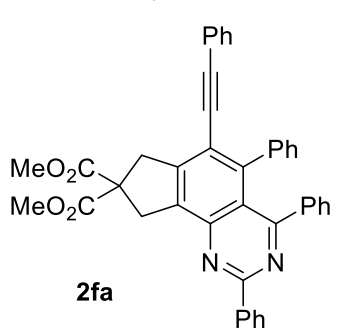

(SiO, EtOAc-hexanes, 1:3) as white solid (44\% yield, $31.4 \mathrm{mg}$ ) after stirring the reaction mixture for $2 \mathrm{~h} .{ }^{1} \mathbf{H}$ NMR $\left(\mathrm{CDCl}_{3}, 500 \mathrm{MHz}\right): \delta 8.74-8.69(\mathrm{~m}, 2 \mathrm{H})$, 7.56-7.48 (m, 3H), 7.31-7.22 (m, 7H), 7.19-7.14 (m, 2H), 7.11-7.00 (m, 6H), $4.36(\mathrm{~s}, 2 \mathrm{H}), 4.08(\mathrm{~s}, 2 \mathrm{H}), 3.86(\mathrm{~s}, 6 \mathrm{H}) ;{ }^{13} \mathrm{C}$ NMR $\left(\mathrm{CDCl}_{3}, 125 \mathrm{MHz}\right): \delta 172.2$, $168.8,158.9,149.3,146.4,142.6,140.4,138.8,137.6,136.7,131.9,131.5$, $130.7,130.4,130.0,128.8,128.7,128.5,128.4,128.3,127.3,127.2,127.1$, 123.0, 120.7, 120.0, 97.8, 87.2, 59.0, 53.3, 42.7, 40.0; HRMS (ESI) calcd for $\mathrm{C}_{41} \mathrm{H}_{31} \mathrm{~N}_{2} \mathrm{O}_{4}[\mathrm{M}+\mathrm{H}]^{+}$615.2284, found 615.2299.

2f'a: This product was synthesized using procedure $\mathbf{A}$ and isolated by flash column chromatography (SiO, EtOAc-hexanes, 1:3) as light yellow-green solid in an inseparable mix with $\mathbf{2 f a}$ (69\% overall yield, $49.2 \mathrm{mg})$ after stirring the reaction mixture for $2 \mathrm{~h} .{ }^{1} \mathbf{H}$ NMR $\left(\mathrm{CDCl}_{3}, 500 \mathrm{MHz}\right): 8.49-8.40(\mathrm{~m}$, $2 \mathrm{H}), 7.80-7.72(\mathrm{~m}, 2 \mathrm{H}), 7.66-7.56(\mathrm{~m} 7 \mathrm{H}), 7.56-7.21(\mathrm{~m}, 9 \mathrm{H}), 3.92(\mathrm{~s}, 2 \mathrm{H}), 3.72(\mathrm{~s}, 6 \mathrm{H}), 3.23(\mathrm{~s}, 2 \mathrm{H})$;

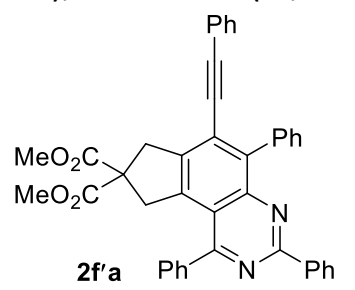

${ }^{13} \mathrm{C} \mathrm{NMR}\left(\mathrm{CDCl}_{3}, 125 \mathrm{MHz}\right): \delta$ (all discernible signals for both isomers) 172.2, $171.8,168.8,167.8,158.9,158.5,150.4,149.3,146.5,142.6,142.5,140.8$, $140.5,140.4,138.8,137.9,137.6,137.0,136.7,134.8,131.9,131.8,131.5$, $130.8,130.4,130.0,129.6,129.1,128.9,128.8,128.7,128.6,128.5,128.4$, $128.3,127.6,127.3,127.2$, 127.1, 124.6, 123.0, 122.7, 120.7, 120.0, 119.5, 99.4, 97.8, 87.2, 87.1, 59.4, 59.0, 53.3, 53.1, 42.9, 42.7, 41.2, 40.0; HRMS (ESI) calcd for $\mathrm{C}_{41} \mathrm{H}_{31} \mathrm{~N}_{2} \mathrm{O}_{4}[\mathrm{M}+\mathrm{H}]^{+} 615.2284$, found 615.2269 . 
2ga: This product was synthesized using procedure $\mathbf{A}$ (20 wt $\% 4 \AA$ MS was used as additive) and isolated by flash column chromatography ( $\mathrm{SiO}$, EtOAc-hexanes, 1:5) as white solid (53\% yield, 36.5

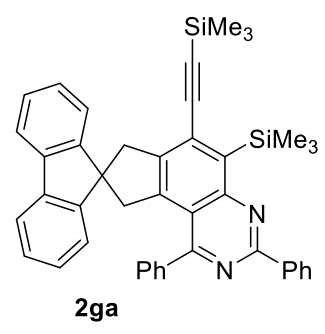
$\mathrm{mg}$ ) after stirring the reaction mixture for $5 \mathrm{~h} .{ }^{1} \mathbf{H}$ NMR $\left(\mathrm{CDCl}_{3}, 500 \mathrm{MHz}\right): \delta$ $8.68(\mathrm{~d}, 2 \mathrm{H}, J=7.0 \mathrm{~Hz}), 7.68(\mathrm{~d}, 2 \mathrm{H}, J=7.6 \mathrm{~Hz}), 7.57-7.45(\mathrm{~m}, 5 \mathrm{H}), 7.36-7.29$ $(\mathrm{m}, 5 \mathrm{H}), 7.20(\mathrm{t}, 2 \mathrm{H}, J=7.4 \mathrm{~Hz}), 7.17-7.13(\mathrm{~m}, 2 \mathrm{H}), 3.60(\mathrm{~s}, 2 \mathrm{H}), 3.03(\mathrm{~s}, 2 \mathrm{H})$, $0.72(\mathrm{~s}, 9 \mathrm{H}), 0.20(\mathrm{~s}, 9 \mathrm{H}) ;{ }^{13} \mathrm{C}$ NMR $\left(\mathrm{CDCl}_{3}, 125 \mathrm{MHz}\right): \delta 168.2,157.2,156.9$, $152.2,145.2,142.1,140.6,139.5,139.2,138.2,132.0,130.3,129.4,129.1$, $128.7,128.5,128.1,127.7,127.4,122.3,119.8,119.3,105.6,104.0,48.1$, 46.2, 3.1, -0.3 ; HRMS (ESI) calcd for $\mathrm{C}_{43} \mathrm{H}_{41} \mathrm{~N}_{2} \mathrm{Si}_{2}[\mathrm{M}+\mathrm{H}]^{+} 641.2808$, found 641.2799 .

2ha: This product was synthesized using procedure $\mathbf{A}$ (20 wt\% $4 \AA$ MS was used as additive) and isolated by flash column chromatography (SiO, EtOAc-hexanes, 1:10) as colourless solid (58\% yield,<smiles>COc1c(C#CSC)c2c(c3c(-c4ccccc4)nc(-c4ccccc4)nc13)C[N]C2</smiles>
$44.1 \mathrm{mg})$ after stirring the reaction mixture for $30 \mathrm{~min} .{ }^{1} \mathrm{H} \mathbf{~ N M R}\left(\mathrm{CDCl}_{3}, 500 \mathrm{MHz}\right)$ : 8 8.63-8.53 (m, 2H), $7.65(\mathrm{t}, 1 \mathrm{H}, J=7.5 \mathrm{~Hz}), 7.61-7.53(\mathrm{~m}, 4 \mathrm{H}), 7.53-7.43(\mathrm{~m}, 3 \mathrm{H})$, $7.41(\mathrm{~d}, 2 \mathrm{H}, J=7.2 \mathrm{~Hz}), 7.29(\mathrm{~d}, 2 \mathrm{H}, J=8.1 \mathrm{~Hz}), 4.69(\mathrm{~s}, 2 \mathrm{H}), 4.05(\mathrm{~s}, 2 \mathrm{H}), 2.39(\mathrm{~s}$, $3 \mathrm{H}), 0.61(\mathrm{~s}, 9 \mathrm{H}), 0.31(\mathrm{~s}, 9 \mathrm{H}) ;{ }^{13} \mathrm{C} \mathrm{NMR}\left(\mathrm{CDCl}_{3}, 125 \mathrm{MHz}\right): \delta 167.7,158.0,156.7$, $143.8,143.5,139.5,138.8,137.7,133.3,132.9,130.7,130.0,129.8,128.8,128.6$, 128.4, 127.6, 117.2, 106.9, 102.6, 55.7, 54.7, 29.7, 21.5, 2.7, -0.3; HRMS (ESI) calcd for $\mathrm{C}_{37} \mathrm{H}_{40} \mathrm{~N}_{3} \mathrm{O}_{2} \mathrm{SSi}_{2}[\mathrm{M}+\mathrm{H}]^{+} 646.2380$, found 646.2390 .

2ia: This product was synthesized using procedure $\mathbf{A}$ (20 wt\% $4 \AA$ MS was used as additive) and isolated by flash column chromatography (SiO, EtOAc-hexanes, $1: 10$ ) as red-yellow solid (36\% yield,

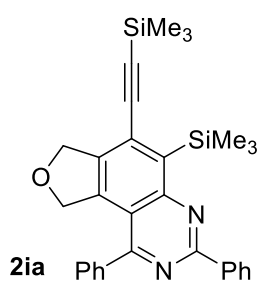
$26.5 \mathrm{mg})$ after stirring the reaction mixture for $30 \mathrm{~min} .{ }^{1} \mathbf{H} \mathbf{~ N M R}\left(\mathrm{CDCl}_{3}, 500 \mathrm{MHz}\right)$ : ס 8.67-8.60 (m, 2H), 7.60-7.42 (m, 8H), 5.22-5.12 (m, 2H), 4.61-4.53 (m, 2H), $0.66(\mathrm{~s}, 9 \mathrm{H}), 0.29(\mathrm{~s}, 9 \mathrm{H}) ;{ }^{13} \mathrm{C}$ NMR $\left(\mathrm{CDCl}_{3}, 125 \mathrm{MHz}\right): \delta 172.2,168.8,158.9,149.3$, $146.4,142.6,140.4,138.8,137.6,136.7,131.9,131.5,130.7,130.4,130.0,128.8$, $128.7,128.5,128.4,128.3,127.3,127.2,127.1,123.0,120.7,120.0,97.8,87.2$, 59.0, 53.3, 42.7, 40.0; HRMS (ESI) calcd for $\mathrm{C}_{30} \mathrm{H}_{33} \mathrm{~N}_{2} \mathrm{OSi}{ }_{2}[\mathrm{M}+\mathrm{H}]^{+} 493.2131$, found 493.2130 .

2ja: This product was synthesized using procedure $\mathbf{A}$ (20 wt\% $4 \AA$ MS was used as additive) and isolated by flash column chromatography (SiO, EtOAc-hexanes, $1: 10$ ) as white solid (48\% yield, 33.0 $\mathrm{mg}$ ) after stirring the reaction mixture for $30 \mathrm{~min} .{ }^{1} \mathrm{H} \mathrm{NMR}\left(\mathrm{CDCl}_{3}, 500 \mathrm{MHz}\right): \delta 8.71(\mathrm{~d}, 2 \mathrm{H}, J=8.0$<smiles></smiles>
$\mathrm{Hz}), 7.87(\mathrm{~d}, 2 \mathrm{H}, \mathrm{J}=7.0 \mathrm{~Hz}), 7.58-7.47(\mathrm{~m}, 6 \mathrm{H}), 7.07(\mathrm{~d}, 2 \mathrm{H}, \mathrm{J}=8.1 \mathrm{~Hz}), 6.89(\mathrm{~d}, 2 \mathrm{H}$, $J=8.0 \mathrm{~Hz}), 3.96-3.78(\mathrm{~m}, 2 \mathrm{H}), 2.78-2.68(\mathrm{~m}(\mathrm{~m}, 1 \mathrm{H}), 2.45(\mathrm{t}, 2 \mathrm{H}, J=7.1 \mathrm{~Hz}), 2.37$ $(\mathrm{s}, 3 \mathrm{H}), 2.36-2.28(\mathrm{~m}, 1 \mathrm{H}), 1.62-1.54(\mathrm{~m} 2 \mathrm{H}), 1.49-1.40(\mathrm{~m}, 2 \mathrm{H}), 0.94(\mathrm{t}, 3 \mathrm{H}), 0.65$ (s, 9H); ${ }^{13} \mathrm{C}$ NMR $\left(\mathrm{CDCl}_{3}, 125 \mathrm{MHz}\right): \delta 144.2,141.1,140.2,139.0,138.1,138.0$, $133.8,131.7,130.7,130.4,129.5,129.4,128.8,128.5,127.8,127.4,114.6,101.1$, 79.8, 51.1, 30.4, 30.1, 22.1, 21.5, 19.5, 13.6, 2.8; HRMS (ESI) calcd for $\mathrm{C}_{38} \mathrm{H}_{40} \mathrm{~N}_{3} \mathrm{O}_{2} \mathrm{SSi}[\mathrm{M}+\mathrm{H}]^{+} 630.2611$, found 630.2615 .

2ka: This product was synthesized using procedure $\mathbf{A}$ (20 wt\% $4 \AA$ MS was used as additive) and isolated by flash column chromatography (SiO, EtOAc-hexanes, 1:10) as colourless solid (70\% yield, $34.8 \mathrm{mg})$ after stirring the reaction mixture for $5 \mathrm{~h} .{ }^{1} \mathrm{H}$ NMR $\left(\mathrm{CDCl}_{3}, 500 \mathrm{MHz}\right): \delta$<smiles>C[As](C)c1cc2c(c(-c3ccccc3)n1)COC2=O</smiles>
8.73-8.68 (m, 2H), $8.41(\mathrm{~s}, 1 \mathrm{H}), 7.70-7.59(\mathrm{~m}, 3 \mathrm{H}), 7.59-7.51(\mathrm{~m}, 5 \mathrm{H}), 4.75(\mathrm{~s}$, $2 \mathrm{H}), 0.58(\mathrm{~s}, 9 \mathrm{H}) ;{ }^{13} \mathrm{C}$ NMR $\left(\mathrm{CDCl}_{3}, 125 \mathrm{MHz}\right): \delta 170.5,168.9,160.9,159.1,147.9$, 145.0, 139.0, 137.4, 135.1, 131.4, 130.4, 129.1, 129.0, 128.8, 128.1, 124.2, 117.1, 69.9, -0.2; HRMS (ESI) calcd for $\mathrm{C}_{25} \mathrm{H}_{23} \mathrm{~N}_{2} \mathrm{O}_{2} \mathrm{Si}[\mathrm{M}+\mathrm{H}]^{+} 411.1529$, found 411.1519. 
2la: This product was synthesized using procedure $\mathbf{A}$ and isolated by flash column chromatography (SiO, EtOAc-hexanes, 1:5) as light yellow solid (76\% yield, $42.9 \mathrm{mg}$ ) after stirring the reaction mixture for $2 \mathrm{~h} .{ }^{1} \mathrm{H}$ NMR $\left(\mathrm{CDCl}_{3}, 500 \mathrm{MHz}\right): 8.73-8.66(\mathrm{~m}, 2 \mathrm{H}), 8.47(\mathrm{~s}, 1 \mathrm{H}), 7.74-7.59(\mathrm{~m}, 5 \mathrm{H}), 7.59-7.50(\mathrm{~m}$,<smiles>CCSc1cc2c(c(-c3ccccc3)n1)CNC(=O)C2</smiles>
$5 \mathrm{H}), 7.36(\mathrm{t}, 2 \mathrm{H}, J=7.9 \mathrm{~Hz}), 7.36(\mathrm{t}, 1 \mathrm{H}, J=7.9 \mathrm{~Hz}), 4.30(\mathrm{~s}, 2 \mathrm{H}), 1.20(\mathrm{q}, 6 \mathrm{H}, J=7.8$ $\mathrm{Hz}), 1.00(\mathrm{t}, 9 \mathrm{H}, J=7.8 \mathrm{~Hz}) ;{ }^{13} \mathrm{C}$ NMR $\left(\mathrm{CDCl}_{3}, 125 \mathrm{MHz}\right): \delta 168.6,166.6,159.9$, 158.6, 141.2, 140.1, 139.8, 139.1, 137.8, 136.0, 131.8, 131.0, 130.0, 129.2, 129.0, $128.7,128.2,124.5,118.9,117.4,51.4,7.7,4.0 ;$ HRMS (ESI) calcd for $\mathrm{C}_{34} \mathrm{H}_{34} \mathrm{~N}_{3} \mathrm{OSi}$ $[\mathrm{M}+\mathrm{H}]^{+} 528.2471$, found 528.2460 .

2ab: This product was synthesized using procedure $\mathbf{B}$ and isolated by flash column chromatography (SiO, EtOAc-hexanes, 1:5) as light yellow solid (48\% yield, $34.4 \mathrm{mg}$ ) after stirring the reaction mixture for 2 h. ${ }^{1} \mathrm{H}$ NMR $\left(\mathrm{CDCl}_{3}, 500 \mathrm{MHz}\right): \delta 8.56(\mathrm{~d}, 2 \mathrm{H}, J=8.8 \mathrm{~Hz}), 7.50(\mathrm{~d}, 2 \mathrm{H}, J=$ 2ab $8.6 \mathrm{~Hz}), 7.06(\mathrm{~d}, 2 \mathrm{H}, J=8.5 \mathrm{~Hz}), 7.01(\mathrm{~d}, 2 \mathrm{H}, J=8.1 \mathrm{~Hz}), 3.92(\mathrm{~s}, 3 \mathrm{H}), 3.88(\mathrm{~s}$, $3 \mathrm{H}), 3.73(\mathrm{~s}, 2 \mathrm{H}), 3.70(\mathrm{~s}, 6 \mathrm{H}), 3.25(\mathrm{~s}, 2 \mathrm{H}), 0.62(\mathrm{~s}, 9 \mathrm{H}), 0.31(\mathrm{~s}, 9 \mathrm{H}) ;{ }^{13} \mathrm{C}$ NMR $\left(\mathrm{CDCl}_{3}, 125 \mathrm{MHz}\right): \delta 171.9,167.3,161.5,160.8,157.1,157.0,141.9,141.7$, $136.4,133.1,131.2,130.9,130.8,130.2,118.5,113.8,113.7,105.5,103.9$, $59.2,55.4,53.0,43.3,41.4,3.0,-0.2$; HRMS (ESI) calcd for $\mathrm{C}_{37} \mathrm{H}_{43} \mathrm{~N}_{2} \mathrm{O}_{6} \mathrm{Si}_{2}$ $[\mathrm{M}+\mathrm{H}]^{+} 667.2660$, found 667.2664 .

2ac: This product was synthesized using procedure $\mathbf{B}$ and isolated by flash column chromatography (SiO, EtOAc-hexanes, 1:5) as yellow-green solid (48\% yield, $36.0 \mathrm{mg}$ ) after stirring the reaction<smiles>CC#Cc1c2c(c(-c3ccc(N(C)C)cc3)c3nc(-c4ccc(N(C)C)cc4)nc(-c4ccc([N+](=O)[O-])cc4)c13)CC(C)(C(C)=O)C2</smiles>
mixture for $2 \mathrm{~h} .{ }^{1} \mathrm{H}$ NMR $\left(\mathrm{CDCl}_{3}, 500 \mathrm{MHz}\right): \delta 8.51(\mathrm{~d}, 2 \mathrm{H}, J=8.9 \mathrm{~Hz})$, $7.47(\mathrm{~d}, 2 \mathrm{H}, J=8.7 \mathrm{~Hz}), 6.82(\mathrm{~d}, 2 \mathrm{H}, J=8.8 \mathrm{~Hz}), 6.79(\mathrm{~d}, 2 \mathrm{H}, J=9.0$ $\mathrm{Hz}), 3.72(\mathrm{~s}, 2 \mathrm{H}), 3.70(\mathrm{~s}, 6 \mathrm{H}), 3.37(\mathrm{~s}, 2 \mathrm{H}), 3.07(\mathrm{~s}, 6 \mathrm{H}), 3.05(\mathrm{~s}, 6 \mathrm{H})$, $0.61(\mathrm{~s}, 9 \mathrm{H}), 0.30(\mathrm{~s}, 9 \mathrm{H}) ;{ }^{13} \mathrm{C}$ NMR $\left(\mathrm{CDCl}_{3}, 125 \mathrm{MHz}\right): \delta$ 172.1, 167.4, $157.5,157.4,151.8,151.4,141.4,140.6,136.6,130.8,130.6,130.0$, $128.5,126.4,118.3,111.7,111.6,104.6,104.2,59.3,52.9,43.5$, 41.4, 40.4, 40.3, 3.0; HRMS (ESI) calcd for $\mathrm{C}_{39} \mathrm{H}_{49} \mathrm{~N}_{4} \mathrm{O}_{4} \mathrm{Si}_{2}[\mathrm{M}+\mathrm{H}]^{+}$ 693.3292, found 693.3278 .

2ad: This product was synthesized using procedure $\mathbf{B}$ and isolated by flash column chromatography (SiO, EtOAc-hexanes, 1:10) as white solid (65\% yield, $38.8 \mathrm{mg}$ ) after stirring the reaction mixture for 2 h. ${ }^{1} \mathrm{H}$ NMR $\left(\mathrm{CDCl}_{3}, 500 \mathrm{MHz}\right): \delta 8.60(\mathrm{dd}, 2 \mathrm{H}, J=8.9 \mathrm{~Hz}, J=5.6 \mathrm{~Hz}), 7.54(\mathrm{dd}, 2 \mathrm{H}, J=8.5 \mathrm{~Hz}, J=5.4$

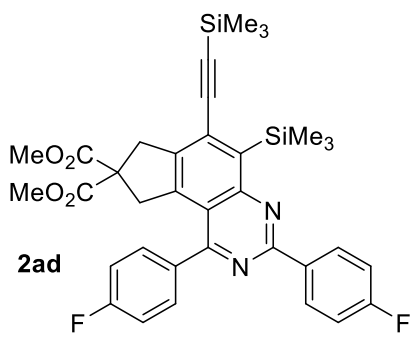
$\mathrm{Hz}), 7.24(\mathrm{t}, 2 \mathrm{H}, J=8.5 \mathrm{~Hz}), 7.18(\mathrm{t}, 2 \mathrm{H}, J=8.7 \mathrm{~Hz}), 3.74(\mathrm{~s}, 2 \mathrm{H}), 3.71(\mathrm{~s}$, $6 \mathrm{H}), 3.17(\mathrm{~s}, 2 \mathrm{H}), 0.62(\mathrm{~s}, 9 \mathrm{H}), 0.31(\mathrm{~S}, 9 \mathrm{H}) ;{ }^{13} \mathrm{C} \mathrm{NMR}\left(\mathrm{CDCl}_{3}, 125 \mathrm{MHz}\right): \delta$ $171.8,166.9,164.5(\mathrm{~d}, J=250.7 \mathrm{~Hz}), 163.7(\mathrm{~d}, J=249.4 \mathrm{~Hz}), 156.9$, $156.4,142.5,142.3,136.6(\mathrm{~d}, J=2.8 \mathrm{~Hz}), 136.1,134.1(\mathrm{~d}, J=2.3 \mathrm{~Hz})$, $131.8,131.1(\mathrm{~d}, J=8.3 \mathrm{~Hz}), 130.6(\mathrm{~d}, J=8.5 \mathrm{~Hz}), 118.6,115.5(\mathrm{~d}, J=21.8$ $\mathrm{Hz}), 106.3,103.7,59.2,53.1,43.2,41.4,2.9,-0.3 ;{ }^{19} \mathrm{~F}$ NMR $(375 \mathrm{MHz}$, $\mathrm{CDCl}_{3}$ ): $\delta-111.2,-111.7$; HRMS (ESI) calcd for $\mathrm{C}_{35} \mathrm{H}_{37} \mathrm{~F}_{2} \mathrm{~N}_{2} \mathrm{O}_{4} \mathrm{Si}_{2}[\mathrm{M}+\mathrm{H}]^{+}$ 643.2260 , found 643.2231 .

2ae: This product was synthesized using procedure $\mathbf{B}$ and isolated by flash column chromatography

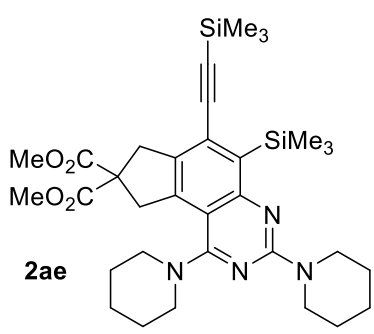
(SiO, EtOAc-hexanes, 1:5) as yellow solid ( $83 \%$ yield, $45.2 \mathrm{mg}$ ) after stirring the reaction mixture for $2 \mathrm{~h} .{ }^{1} \mathbf{H}$ NMR $\left(\mathrm{CDCl}_{3}, 500 \mathrm{MHz}\right): \delta 3.95(\mathrm{~s}, 2 \mathrm{H})$, $3.87-3.80(\mathrm{~m}, 4 \mathrm{H}), 3.76(\mathrm{~s}, 6 \mathrm{H}), 3.64(\mathrm{~s}, 2 \mathrm{H}), 3.57-3.39(\mathrm{br}, 2 \mathrm{H}), 3.01-2.85$ (br, 2H), 1.89-1.37 (m, 12H), $0.46(\mathrm{~s}, 9 \mathrm{H}), 0.26(\mathrm{~s}, 9 \mathrm{H}) ;{ }^{13} \mathrm{C} \mathrm{NMR}\left(\mathrm{CDCl}_{3}, 125\right.$ $\mathrm{MHz}): \delta 172.4,167.9,159.8,156.1,136.8,135.9,135.4,129.1,109.6$, 
104.8, 103.0, 59.3, 53.0, 52.2, 45.1, 42.5, 40.8, 26.0, 25.6, 25.2, 24.6, 2.4, -0.2; HRMS (ESI) calcd for $\mathrm{C}_{33} \mathrm{H}_{49} \mathrm{~N}_{4} \mathrm{O}_{4} \mathrm{Si}_{2}[\mathrm{M}+\mathrm{H}]^{+} 621.3292$, found 621.3275 .

2af: This product was synthesized using procedure $\mathbf{B}$ and isolated by flash column chromatography

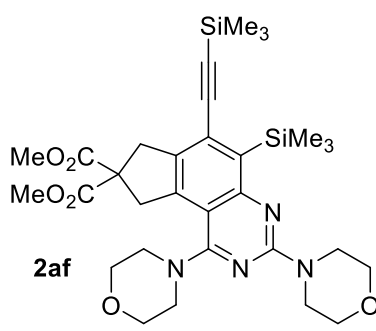
(SiO, EtOAc-hexanes, 1:5) as yellow-green solid ( $70 \%$ yield, $43.7 \mathrm{mg}$ ) after stirring the reaction mixture for $2 \mathrm{~h} .{ }^{1} \mathrm{H}$ NMR $\left(\mathrm{CDCl}_{3}, 500 \mathrm{MHz}\right): \delta 4.00-$ $3.70(\mathrm{~m}, 2 \mathrm{OH}), 3.66(\mathrm{~s}, 2 \mathrm{H}), 3.56-3.06(\mathrm{br}, 4 \mathrm{H}), 0.46(\mathrm{~s}, 9 \mathrm{H}), 0.27(\mathrm{~s}, 9 \mathrm{H})$; ${ }^{13} \mathrm{C}$ NMR $\left(\mathrm{CDCl}_{3}, 125 \mathrm{MHz}\right): \delta 172.2,167.4,159.6,156.0,137.5,136.6$, 135.4, 130.0, 109.6, 104.3, 104.0, 67.0, 66.5, 59.3, 53.1, 51.5, 44.7, 42.6, 40.8, 2.4, -0.2; HRMS (ESI) calcd for $\mathrm{C}_{31} \mathrm{H}_{45} \mathrm{~N}_{4} \mathrm{O}_{6} \mathrm{Si}_{2}[\mathrm{M}+\mathrm{H}]^{+} 625.2878$, found 625.2860 .

2ag: This product was synthesized using procedure $\mathbf{B}$ and isolated by flash column chromatography (SiO, EtOAc-hexanes, 1:10) as orange-yellow solid (40\% yield, $28.6 \mathrm{mg}$ ) after stirring the reaction

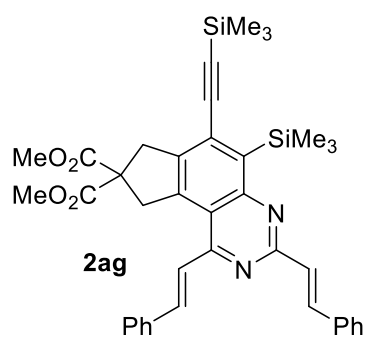
mixture for 2 h. ${ }^{1} \mathbf{H}$ NMR $\left(\mathrm{CDCl}_{3}, 500 \mathrm{MHz}\right): \delta 8.14(\mathrm{~d}, 1 \mathrm{H}, J=7.7 \mathrm{~Hz}), 8.11$ (d, $1 \mathrm{H}, J=7.1 \mathrm{~Hz}), 7.83(\mathrm{~d}, 1 \mathrm{H}, J=15.4 \mathrm{~Hz}), 7.73-7.66(\mathrm{~m}, 4 \mathrm{H}), 7.50-7.32(\mathrm{~m}$, 7H), $4.26(\mathrm{~s}, 2 \mathrm{H}), 3.82(\mathrm{~s}, 2 \mathrm{H}), 3.80(\mathrm{~s}, 6 \mathrm{H}), 0.58(\mathrm{~s}, 9 \mathrm{H}), 0.31(\mathrm{~s}, 9 \mathrm{H}) ;{ }^{13} \mathrm{C}$ NMR $\left(\mathrm{CDCl}_{3}, 125 \mathrm{MHz}\right): \delta 171.9,162.1,158.1,157.3,142.6,141.9,138.7$, $137.4,136.6,136.3,135.1,131.2,129.3,129.0,128.8,128.5,128.0,127.6$, 125.3, 118.9, 105.7, 103.8, 59.3, 53.3, 44.5, 41.3, 2.9, -0.3; HRMS (ESI) calcd for $\mathrm{C}_{39} \mathrm{H}_{43} \mathrm{~N}_{2} \mathrm{O}_{4} \mathrm{Si}_{2}[\mathrm{M}+\mathrm{H}]^{+}$659.2761, found 659.2780.

2ah: This product was synthesized using procedure $\mathbf{A}$ and isolated by flash column chromatography (SiO, EtOAc-hexanes, 1:10) as orange-red solid (54\% yield, $22.9 \mathrm{mg}$ ) after stirring the reaction mixture for 2 h. ${ }^{1} \mathbf{H}$ NMR $\left(\mathrm{CDCl}_{3}, 500 \mathrm{MHz}\right): \delta 7.51$ (dd, $\left.1 \mathrm{H}, J=16.6 \mathrm{~Hz}, J=10.6 \mathrm{~Hz}\right), 6.98(\mathrm{dd}, 1 \mathrm{H}, J=$

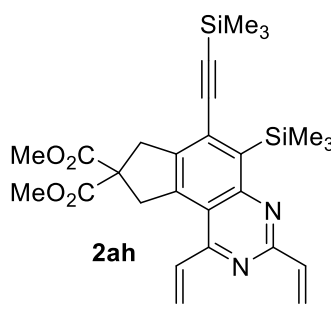
$17.2 \mathrm{~Hz}, J=10.4 \mathrm{~Hz}$ ), $6.70(\mathrm{dd}, 1 \mathrm{H}, J=17.2 \mathrm{~Hz}, J=1.6 \mathrm{~Hz}), 6.60(\mathrm{dd}, 1 \mathrm{H}, J=$ $16.6 \mathrm{~Hz}, J=1.5 \mathrm{~Hz}$ ), 5.77 (dd, $1 \mathrm{H}, J=10.8 \mathrm{~Hz}, J=1.4 \mathrm{~Hz}), 5.73(\mathrm{dd}, 1 \mathrm{H}, J=10.6$ $\mathrm{Hz}, J=1.4 \mathrm{~Hz}), 4.14(\mathrm{~s}, 2 \mathrm{H}), 3.80(\mathrm{~s}, 6 \mathrm{H}), 3.79(\mathrm{~s}, 2 \mathrm{H}), 0.51(\mathrm{~s}, 9 \mathrm{H}), 0.30(\mathrm{~s}, 9 \mathrm{H})$; ${ }^{13} \mathrm{C}$ NMR $\left(\mathrm{CDCl}_{3}, 125 \mathrm{MHz}\right): \delta 171.9,162.4,157.7,157.0,142.7,142.1,137.2$, 135.1, 134.8, 131.2, 124.6, 123.0, 118.6, 105.8, 103.7, 59.1, 53.2, 44.5, 41.2, 2.8, -0.3; HRMS (ESI) calcd for $\mathrm{C}_{27} \mathrm{H}_{35} \mathrm{~N}_{2} \mathrm{O}_{4} \mathrm{Si}_{2}[\mathrm{M}+\mathrm{H}]^{+} 507.2135$, found 507.2129 .

2ai: This product was synthesized using procedure $\mathbf{A}$ and isolated by flash column chromatography

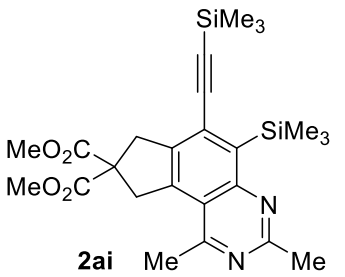
(SiO, EtOAc-hexanes, 1:10) as white solid (66\% yield, $30.4 \mathrm{mg}$ ) after stirring the reaction mixture for $2 \mathrm{~h} .{ }^{1} \mathrm{H}$ NMR $\left(\mathrm{CDCl}_{3}, 500 \mathrm{MHz}\right): \delta 4.16(\mathrm{~s}, 2 \mathrm{H}), 3.80(\mathrm{~s}$, $6 \mathrm{H}), 3.78(\mathrm{~s}, 2 \mathrm{H}), 2.96(\mathrm{~s}, 3 \mathrm{H}), 2.73(\mathrm{~s}, 3 \mathrm{H}), 0.49(\mathrm{~s}, 9 \mathrm{H}), 0.29(\mathrm{~s}, 9 \mathrm{H}) ;{ }^{13} \mathrm{C}$ NMR $\left(\mathrm{CDCl}_{3}, 125 \mathrm{MHz}\right): \delta 172.0,166.6,160.8,155.9,142.3,141.1,135.7,130.8$, 119.4, 105.5, 103.8, 59.0, 53.2, 44.3, 41.1, 26.6, 25.7, 2.7, -0.3; HRMS (ESI) calcd for $\mathrm{C}_{25} \mathrm{H}_{35} \mathrm{~N}_{2} \mathrm{O}_{4} \mathrm{Si}_{2}[\mathrm{M}+\mathrm{H}]^{+} 483.2135$, found 483.2146 .

2aj: This product was synthesized using procedure $\mathbf{A}$ and isolated by flash column chromatography

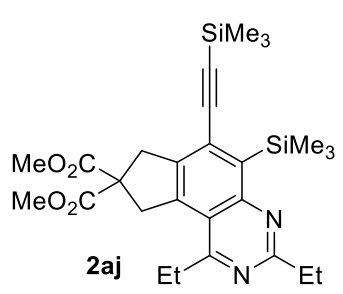
(SiO, EtOAc-hexanes, 1:10) as light yellow solid (69\% yield, $33.2 \mathrm{mg}$ ) after stirring the reaction mixture for $2 \mathrm{~h} .{ }^{1} \mathrm{H}$ NMR $\left(\mathrm{CDCl}_{3}, 500 \mathrm{MHz}\right): \delta 4.15(\mathrm{~s}, 2 \mathrm{H})$, 3.83-3.77 (m, 8H), $3.26(\mathrm{q}, 2 \mathrm{H}, J=7.4 \mathrm{~Hz}), 3.05(\mathrm{q}, 2 \mathrm{H}, J=7.5 \mathrm{~Hz}), 1.41(\mathrm{t}, 3 \mathrm{H}, J$

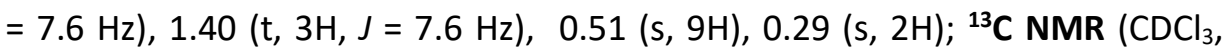
$125 \mathrm{MHz}): \delta 172.0,171.0,164.9,156.3,142.4,141.2,135.1,130.6,118.8$, 105.2, 103.9, 59.1, 53.2, 44.0, 41.1, 32.6, 31.3, 13.7, 12.5, 2.8; HRMS (ESI) 
calcd for $\mathrm{C}_{27} \mathrm{H}_{39} \mathrm{~N}_{2} \mathrm{O}_{4} \mathrm{Si}_{2}[\mathrm{M}+\mathrm{H}]^{+}$511.2448, found 511.2453 .

2ak: This product was synthesized using procedure $\mathbf{A}$ and isolated by flash column chromatography

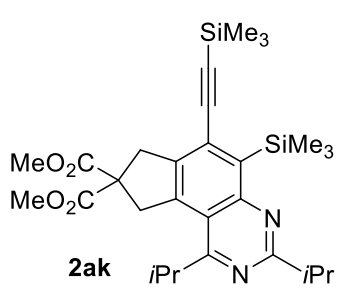
(SiO, EtOAc-hexanes, 1:10) as white solid ( $74 \%$ yield, $37.9 \mathrm{mg}$ ) after stirring the reaction mixture for $2 \mathrm{~h} .{ }^{1} \mathrm{H}$ NMR $\left(\mathrm{CDCl}_{3}, 500 \mathrm{MHz}\right): \delta 4.16(\mathrm{~s}, 2 \mathrm{H}), 3.84-$ $3.74(\mathrm{~m}, 9 \mathrm{H}), 3.28(\mathrm{sept}, 1 \mathrm{H}, \mathrm{J}=6.9 \mathrm{~Hz}), 1.39(\mathrm{~d}, 6 \mathrm{H}, \mathrm{J}=6.78 \mathrm{~Hz}), 1.38(\mathrm{~d}, 6 \mathrm{H}, J$ $=6.4 \mathrm{~Hz}), 0.51(\mathrm{~s}, 9 \mathrm{H}), 0.29(\mathrm{~s}, 9 \mathrm{H}) ;{ }^{13} \mathrm{C}$ NMR $\left(\mathrm{CDCl}_{3}, 125 \mathrm{MHz}\right): \delta$ 174.4, 172.0, $168.0,156.5,142.6,141.2,134.6,130.2,118.5,104.9,104.0,59.2,53.2,44.2$, 41.0, 37.6, 33.4, 22.4, 21.7, 2.9, -0.2; HRMS (ESI) calcd for $\mathrm{C}_{29} \mathrm{H}_{43} \mathrm{~N}_{2} \mathrm{O}_{4} \mathrm{Si}_{2}$ $[\mathrm{M}+\mathrm{H}]^{+} 539.2761$, found 539.2748 .

2al: This product was synthesized using procedure B (10 equiv cyclopropanecarbonitrile was used) and isolated by flash column chromatography (SiO, EtOAc-hexanes, 1:10) as white solid (87\% yield, 2al $40.5 \mathrm{mg})$ after stirring the reaction mixture for $2 \mathrm{~h} .{ }^{1} \mathbf{H}$ NMR $\left(\mathrm{CDCl}_{3}, 500\right.$ $\mathrm{MHz}): \delta 4.27(\mathrm{~s}, 2 \mathrm{H}), 3.79(\mathrm{~s}, 6 \mathrm{H}), 3.77(\mathrm{~s}, 2 \mathrm{H}), 2.64-2.56(\mathrm{~m}, 1 \mathrm{H}), 2.25-2.18$ $(\mathrm{m}, 1 \mathrm{H}), 1.38-1.34(\mathrm{~m}, 2 \mathrm{H}), 1.18-1.09(\mathrm{~m}, 4 \mathrm{H}), 1.01-0.95(\mathrm{~m}, 2 \mathrm{H}), 0.48(\mathrm{~s}$, 9H), 0.29 (s, 9H); ${ }^{13} \mathrm{C}$ NMR ( $\left.\mathrm{CDCl}_{3}, 125 \mathrm{MHz}\right): \delta$ 172.1, 169.9, 164.8, 155.9, 141.6, 140.5, 135.5, 130.4, 119.8, 105.0, 104.1, 59.2, 53.2, 44.4, 41.0, 18.2, 16.8, 11.2, 9.7, 2.8, -0.3; HRMS (ESI) calcd for $\mathrm{C}_{29} \mathrm{H}_{39} \mathrm{~N}_{2} \mathrm{O}_{4} \mathrm{Si}_{2}[\mathrm{M}+\mathrm{H}]^{+}$ 535.2448 , found 535.2433 .

2am: This product was synthesized using procedure $\mathbf{B}$ and isolated by flash column chromatography (SiO, EtOAc-hexanes, 1:10) as white solid ( $46 \%$ yield, $35.2 \mathrm{mg}$ ) after stirring the reaction mixture for<smiles>CC#Cc1c2c(c3c(-c4ccc5ccccc5c4)nc(-c4ccc5ccccc5c4)nc3c1C)CC(C(C)(C)C)C2</smiles>
2 h. ${ }^{1} \mathrm{H}$ NMR $\left(\mathrm{CDCl}_{3}, 500 \mathrm{MHz}\right): \delta 9.18(\mathrm{~s}, 1 \mathrm{H}), 8.74(\mathrm{dd}, 1 \mathrm{H}, J=8.7 \mathrm{~Hz}, J=$ $1.2 \mathrm{~Hz}), 8.02-7.95(\mathrm{~m}, 4 \mathrm{H}), 7.90-7.86(\mathrm{~m}, 1 \mathrm{H}), 7.75(\mathrm{dd}, 1 \mathrm{H}, J=8.4 \mathrm{~Hz}, J=$ $1.2 \mathrm{~Hz}), 7.64-7.57(\mathrm{~m}, 2 \mathrm{H}), 7.55-7.47(\mathrm{~m}, 2 \mathrm{H}), 3.76(\mathrm{~s}, 2 \mathrm{H}), 3.70-3.56(\mathrm{br}$, $6 \mathrm{H}), 3.22(\mathrm{~s}, 2 \mathrm{H}), 0.70(\mathrm{~s}, 9 \mathrm{H}), 0.32(\mathrm{~s}, 9 \mathrm{H}) ;{ }^{13} \mathrm{C}$ NMR $\left(\mathrm{CDCl}_{3}, 125 \mathrm{MHz}\right): \delta$ $157.1,142.5,142.4,138.0,136.4,135.5,134.6,133.8,133.4,132.9,131.7$, $129.3,129.1,128.6,128.2,128.1,128.0,127.7,127.0,126.7,126.1,125.6$, 118.9, 106.0, 103.8, 59.1, 53.0, 43.3, 41.5, 3.0, -0.2; HRMS (ESI) calcd for $\mathrm{C}_{43} \mathrm{H}_{43} \mathrm{~N}_{2} \mathrm{O}_{4} \mathrm{Si}_{2}[\mathrm{M}+\mathrm{H}]^{+}$707.2761, found 707.2772.

2ee: This product was synthesized using procedure B (20 wt\% $4 \AA$ MS was used as additive) and isolated by flash column chromatography (SiO, EtOAc-hexanes, 1:5) as red solid (67\% yield, $41.1 \mathrm{mg}$ ) after stirring the reaction mixture for $2 \mathrm{~h} .{ }^{1} \mathrm{H}$ NMR $\left(\mathrm{CDCl}_{3}, 500 \mathrm{MHz}\right): \delta 7.86(\mathrm{~d}, 2 \mathrm{H}, J=7.5 \mathrm{~Hz}), 7.52$

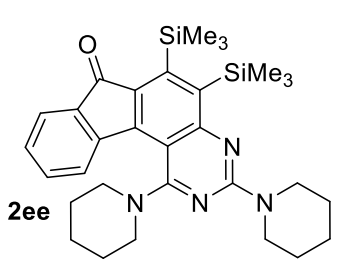
$(\mathrm{d}, 2 \mathrm{H}, J=7.1 \mathrm{~Hz}), 7.38(\mathrm{~d}, 2 \mathrm{H}, J=7.4 \mathrm{~Hz}), 7.23(\mathrm{~d}, 2 \mathrm{H}, J=7.3 \mathrm{~Hz}), 4.00-3.86$ $(\mathrm{m}, 4 \mathrm{H}), 3.83-3.71(\mathrm{~m}, 2 \mathrm{H}), 3.44-3.33(\mathrm{~m}, 2 \mathrm{H}), 1.80-1.49(\mathrm{~m}, 12 \mathrm{H}), 0.43(\mathrm{~s}$, $18 \mathrm{H}) ;{ }^{13} \mathrm{C}$ NMR $\left(\mathrm{CDCl}_{3}, 125 \mathrm{MHz}\right): \delta 194.0,164.7,162.7,157.4,156.2,148.5$, $143.9,143.5,134.4,133.6,132.6,129.0,124.8,122.7,104.9,50.8,45.3,26.1$, 25.3, 25.1, 24.7, 3.3, 2.8; HRMS (ESI) calcd for $\mathrm{C}_{31} \mathrm{H}_{43} \mathrm{~N}_{4} \mathrm{OSi}_{2}[\mathrm{M}+\mathrm{H}]^{+}$543.2975, found 543.2991 .

2ej: This product was synthesized using procedure A (20 wt\% $4 \AA$ MS was used as additive) and isolated by flash column chromatography (SiO, EtOAc-hexanes, 1:10) as orange-red solid (63\% yield,

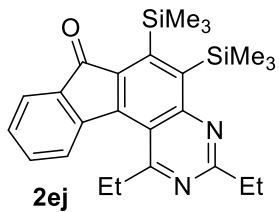
$32.6 \mathrm{mg})$ after stirring the reaction mixture for $2 \mathrm{~h} .{ }^{1} \mathrm{H} \mathrm{NMR}\left(\mathrm{CDCl}_{3}, 500 \mathrm{MHz}\right): \delta$ 7.64-7.60 (m, 1H), 7.55-7.52 (m, 1H), 7.49-7.43 (m, 1H), 7.33-7.27 (m, 1H), 3.33 (q, $2 \mathrm{H}, J=7.4 \mathrm{~Hz}), 3.12(\mathrm{q}, 2 \mathrm{H}, J=7.6 \mathrm{~Hz}), 1.49(\mathrm{t}, 3 \mathrm{H}, J=7.6 \mathrm{~Hz}), 1.36(\mathrm{t}, 3 \mathrm{H}, J=$ $7.4 \mathrm{~Hz}), 0.49(\mathrm{~s}, 9 \mathrm{H}), 0.44(\mathrm{~s}, 9 \mathrm{H}) ;{ }^{13} \mathrm{C} \mathrm{NMR}\left(\mathrm{CDCl}_{3}, 125 \mathrm{MHz}\right): \delta$ 195.0, 171.6, 
$166.9,157.9,155.9,155.1,144.0,143.1,137.3,134.0,133.3,129.4,124.9,123.9,115.9,32.6,31.5$, 13.8, 12.2, 3.6, 2.6; HRMS (ESI) calcd for $\mathrm{C}_{25} \mathrm{H}_{33} \mathrm{~N}_{2} \mathrm{OSi}{ }_{2}[\mathrm{M}+\mathrm{H}]^{+} 433.2131$, found 433.2133 .

2gj: This product was synthesized using procedure $\mathbf{A}$ and isolated by flash column chromatography (SiO, EtOAc-hexanes, 1:10) as white solid (75\% yield, $43.4 \mathrm{mg}$ ) after stirring the reaction mixture for

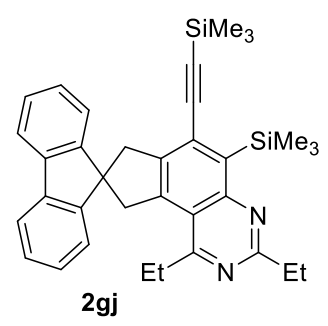
5 h. ${ }^{1} \mathrm{H}$ NMR $\left(\mathrm{CDCl}_{3}, 500 \mathrm{MHz}\right): \delta 7.78(\mathrm{~d}, 1 \mathrm{H}, J=7.6 \mathrm{~Hz}), 7.52(\mathrm{td}, 1 \mathrm{H}, J=7.4$ $\mathrm{Hz}, J=1.1 \mathrm{~Hz}), 7.32-7.23(\mathrm{~m}, 2 \mathrm{H}), 4.00(\mathrm{~s}, 2 \mathrm{H}), 3.67(\mathrm{~s}, 2 \mathrm{H}), 3.12(\mathrm{q}, 2 \mathrm{H}, J=7.5$ $\mathrm{Hz}), 3.09(\mathrm{q}, 2 \mathrm{H}, J=7.6 \mathrm{~Hz}), 1.46(\mathrm{q}, 3 \mathrm{H}, J=7.6 \mathrm{~Hz}), 1.23(\mathrm{q}, 1 \mathrm{H}, J=7.5 \mathrm{~Hz})$, $0.58(\mathrm{~s}, 9 \mathrm{H}), 0.18(\mathrm{~s}, 9 \mathrm{H}) ;{ }^{13} \mathrm{C}$ NMR $\left(\mathrm{CDCl}_{3}, 125 \mathrm{MHz}\right): 171.4,164.8,156.3$, $152.7,144.2,142.1,139.6,137.9,131.1,127.9,127.6,122.4,119.9,119.4$, 105.0, 104.2, 55.9, 49.8, 45.7, 32.6, 31.2, 13.9, 12.5, 3.0, -0.3; HRMS (ESI) calcd for $\mathrm{C}_{35} \mathrm{H}_{41} \mathrm{~N}_{2} \mathrm{Si}_{2}[\mathrm{M}+\mathrm{H}]^{+} 545.2808$, found 545.2785 .

2hl: This product was synthesized using procedure B (10 equiv cyclopropanecarbonitrile was added, 20 wt\% $4 \AA$ MS was used as additive) and isolated by flash column chromatography (SiO, EtOAchexanes, 1:10) as white solid (35\% yield, $20.4 \mathrm{mg}$ ) after stirring the reaction mixture for $30 \mathrm{~min} .{ }^{1} \mathbf{H}$<smiles>CC(C)=Cc1c(C2CC2)nc(C2CC2)c2c1C[NH2+]C2</smiles>
NMR $\left(\mathrm{CDCl}_{3}, 500 \mathrm{MHz}\right): \delta 7.79(\mathrm{~d}, 2 \mathrm{H}, J=8.2 \mathrm{~Hz}), 7.32(\mathrm{~d}, 2 \mathrm{H}, J=8.1 \mathrm{~Hz}), 5.31-$ $5.26(\mathrm{~m}, 2 \mathrm{H}), 4.75-4.70(\mathrm{~m}, 2 \mathrm{H}), 2.40(\mathrm{~s}, 3 \mathrm{H}), 2.29-2.18(\mathrm{~m}, 2 \mathrm{H}), 1.36-1.30(\mathrm{~m}$, 2H), 1.19-1.09 (m, 4H), 1.04-0.96 (m, 2H), $0.46(\mathrm{~s}, 9 \mathrm{H}), 0.29(\mathrm{~s}, 9 \mathrm{H}) ;{ }^{13} \mathrm{C}$ NMR $\left(\mathrm{CDCl}_{3}, 125 \mathrm{MHz}\right): \delta 169.6,165.7,156.1,143.9,142.9,136.9,133.9,131.8,130.0$, $128.7,127.5,118.2,106.0,102.8,57.2,54.4,21.5,18.3,16.1,11.3,10.0,2.6,-$ 0.3 ; HRMS (ESI) calcd for $\mathrm{C}_{31} \mathrm{H}_{40} \mathrm{~N}_{3} \mathrm{O}_{2} \mathrm{SSi}_{2}[\mathrm{M}+\mathrm{H}]^{+} 574.2380$, found 574.2380 .

2ke: This product was synthesized using procedure $\mathbf{B}$ and isolated by flash column chromatography (SiO, EtOAc-hexanes, 1:10) as light yellow solid (59\% yield, $23.7 \mathrm{mg}$ ) after<smiles>C[As](C)(=O)c1cc2c(c(N3CCCCC3)n1)C1COC(=O)C1=C2N1CCCCC1</smiles>
stirring the reaction mixture for $5 \mathrm{~h} .{ }^{1} \mathrm{H}$ NMR $\left(\mathrm{CDCl}_{3}, 500 \mathrm{MHz}\right): \delta 8.04(\mathrm{~s}, 1 \mathrm{H})$, $5.51(\mathrm{~s}, 2 \mathrm{H}), 4.00-3.85(\mathrm{~m}, 4 \mathrm{H}), 3.33-3.10(\mathrm{br}, 4 \mathrm{H}), 1.85-1.55(\mathrm{~m}, 12 \mathrm{H}), 0.38$ $(\mathrm{s}, 9 \mathrm{H}) ;{ }^{13} \mathrm{C}$ NMR $\left(\mathrm{CDCl}_{3}, 125 \mathrm{MHz}\right): \delta 171.6,169.1,163.4,158.3,147.6,139.2$, $134.2,117.5,107.5,70.6,52.5,45.2,26.0,25.7,25.0,24.4,-0.8$; HRMS (ESI) calcd for $\mathrm{C}_{23} \mathrm{H}_{33} \mathrm{~N}_{4} \mathrm{O}_{2} \mathrm{Si}[\mathrm{M}+\mathrm{H}]^{+} 425.2373$, found 423.2379 .

2le: This product was synthesized using procedure $\mathbf{B}$ and isolated by flash column chromatography (SiO, EtOAc-hexanes, 1:5) as orange-yellow solid (65\% yield, $36.4 \mathrm{mg}$ ) after stirring the reaction<smiles>CCCCNC(=O)c1cc2c(N3CCCCC3)nc(N3CCCCC3)nc2c2c1CCCCC2</smiles>
mixture for 2 h. ${ }^{1} \mathbf{H}$ NMR $\left(\mathrm{CDCl}_{3}, 500 \mathrm{MHz}\right): \delta 8.10(\mathrm{~s}, 1 \mathrm{H}), 7.91(\mathrm{~d}, 2 \mathrm{H}, \mathrm{J}=8.1$ $\mathrm{Hz}), 7.48-7.42(\mathrm{~m}, 2 \mathrm{H}), 7.19(\mathrm{t}, 1 \mathrm{H}, J=7.4 \mathrm{~Hz}), 5.12(\mathrm{~s}, 2 \mathrm{H}), 3.96-3.89(\mathrm{~m}, 4 \mathrm{H})$, 3.74-2.75 (br, 4H), 1.92-1.58 (m, 12H), 1.04-0.90 (m, 15H); ${ }^{13} \mathrm{C} \mathrm{NMR}\left(\mathrm{CDCl}_{3}\right.$, $125 \mathrm{MHz}): \delta 169.1,167.7,162.9,157.8,140.0,139.9,135.4,134.8,129.2$, 125.7, 123.8, 118.9, 108.0, 52.6, 52.0, 45.2, 26.1, 25.9, 25.1, 24.5, 7.8, 3.5; HRMS (ESI) calcd for $\mathrm{C}_{32} \mathrm{H}_{44} \mathrm{~N}_{5} \mathrm{OSi}[\mathrm{M}+\mathrm{H}]^{+} 542.3315$, found 542.3295 .

2kc: This product was synthesized using procedure $\mathbf{B}$ and isolated by flash column chromatography (SiO, EtOAc-hexanes, 1:5) as yellow solid ( $51 \%$ yield, $28.3 \mathrm{mg}$ ) after stirring the reaction mixture for 5 h. ${ }^{1} \mathrm{H}$ NMR $\left(\mathrm{CDCl}_{3}, 500 \mathrm{MHz}\right): \delta 8.59(\mathrm{~d}, 2 \mathrm{H}, J=9.0 \mathrm{~Hz}), 8.30(\mathrm{~s}, 1 \mathrm{H}), 7.45(\mathrm{~d}, 1 \mathrm{H}, J=8.6 \mathrm{~Hz}), 6.85$ (d,<smiles></smiles>
$1 \mathrm{H}, J=8.7 \mathrm{~Hz}), 6.81(\mathrm{~d}, 1 \mathrm{H}, J=9.0 \mathrm{~Hz}), 4.94(\mathrm{~s}, 1 \mathrm{H}), 3.10(\mathrm{~s}, 3 \mathrm{H}), 3.09$ (s, 3H), 0.55 (s, 9H).; ${ }^{13} \mathrm{C}$ NMR $\left(\mathrm{CDCl}_{3}, 125 \mathrm{MHz}\right): \delta$ 168.7, 161.2, $159.9,152.5,151.7,148.5,143.7,134.4,130.6,129.8,126.4,125.3$, 122.5, 116.5, 111.8, 111.6, 111.1, 70.6, 40.3, 40.2, 29.7, -0.2; HRMS (ESI) calcd for $\mathrm{C}_{29} \mathrm{H}_{33} \mathrm{~N}_{4} \mathrm{O}_{2} \mathrm{Si}[\mathrm{M}+\mathrm{H}]^{+}$497.2373, found 497.2383. 
$\mathbf{2 k d}$ : This product was synthesized using procedure $\mathbf{B}$ and isolated by flash column chromatography (SiO, EtOAc-hexanes, 1:10) as light yellow solid $(42 \%$ yield, $27.2 \mathrm{mg})$ after stirring the reaction mixture for 5 h. ${ }^{1} \mathbf{H}$ NMR $\left(\mathrm{CDCl}_{3}, 500 \mathrm{MHz}\right): \delta 8.72-8.65(\mathrm{~m}, 2 \mathrm{H}), 8.42(\mathrm{~s}, 1 \mathrm{H}), 7.60-7.53(\mathrm{~m}, 2 \mathrm{H}), 7.33$<smiles>COc1cc2c(c(-c3ccc(F)cc3)n1)NC(c1ccc(F)cc1)=NC(c1ccccc1)=C2</smiles>
$(\mathrm{t}, 2 \mathrm{H}, J=8.0 \mathrm{~Hz}), 7.23(\mathrm{t}, 2 \mathrm{H}, J=8.7 \mathrm{~Hz}), 4.80(\mathrm{~s}, 2 \mathrm{H}), 0.57(\mathrm{~s}, 9 \mathrm{H}) ;{ }^{13} \mathrm{C}$ NMR $\left(\mathrm{CDCl}_{3}, 125 \mathrm{MHz}\right): \delta 170.3,168.0,165.2(\mathrm{~d}, J=252.3 \mathrm{~Hz}), 164.0(\mathrm{~d}, J=251.8$ $\mathrm{Hz}), 160.0,159.2,147.5,145.0,135.4,135.0(\mathrm{~d}, J=2.7 \mathrm{~Hz}), 133.5(\mathrm{~d}, J=2.0$ $\mathrm{Hz}), 131.2(\mathrm{~d}, J=8.7 \mathrm{~Hz}), 130.3(\mathrm{~d}, J=8.3 \mathrm{~Hz}), 124.3,117.0,116.3(\mathrm{~d}, J=$ $21.6 \mathrm{~Hz}), 115.9$ (d, $J=22.0 \mathrm{~Hz}), 69.9,29.7,-0.2 ;{ }^{19} \mathrm{~F} \mathrm{NMR}\left(375 \mathrm{MHz}, \mathrm{CDCl}_{3}\right)$ : $\delta-109.1,-109.7$; HRMS (ESI) calcd for $\mathrm{C}_{25} \mathrm{H}_{21} \mathrm{~F}_{2} \mathrm{~N}_{2} \mathrm{O}_{2} \mathrm{Si}[\mathrm{M}+\mathrm{H}]^{+}$447.1340, found 447.1351 .

2kl: This product was synthesized using procedure B (10 equiv cyclopropanecarbonitrile was added and 20 wt\% $4 \AA$ MS was used as additive) and isolated by flash column chromatography (SiO, EtOAc-hexanes, 1:10) as colourless solid (57\% yield, 25.5 $\mathrm{mg})$ after stirring the reaction mixture for $5 \mathrm{~h} .{ }^{1} \mathbf{H} \mathbf{~ N M R}\left(\mathrm{CDCl}_{3}, 500 \mathrm{MHz}\right): \delta 8.28$ $(\mathrm{s}, 1 \mathrm{H}), 5.80(\mathrm{~s}, 2 \mathrm{H}), 2.38-2.30(\mathrm{~m}, 1 \mathrm{H}) .2 .19-2.10(\mathrm{~m}, 1 \mathrm{H}), 1.50-1.43(\mathrm{~m}, 2 \mathrm{H})$, $1.32-1.18(\mathrm{~m}, 4 \mathrm{H}), 1.15-1.07(\mathrm{~m}, 2 \mathrm{H}), 0.42(\mathrm{~s}, 9 \mathrm{H}) ;{ }^{13} \mathrm{C} \mathrm{NMR}\left(\mathrm{CDCl}_{3}, 125 \mathrm{MHz}\right): \delta$ $171.1,170.8,169.4,158.6,147.4,144.0,134.3,122.5,117.6,70.8,18.8,16.4$, 11.4, 11.2, -0.4; HRMS (ESI) calcd for $\mathrm{C}_{19} \mathrm{H}_{23} \mathrm{~N}_{2} \mathrm{O}_{2} \mathrm{Si}[\mathrm{M}+\mathrm{H}]^{+} 339.1529$, found 339.1532 . 
${ }^{1} \mathrm{H}$ and ${ }^{13} \mathrm{C}$ NMR Spectra of Substrates
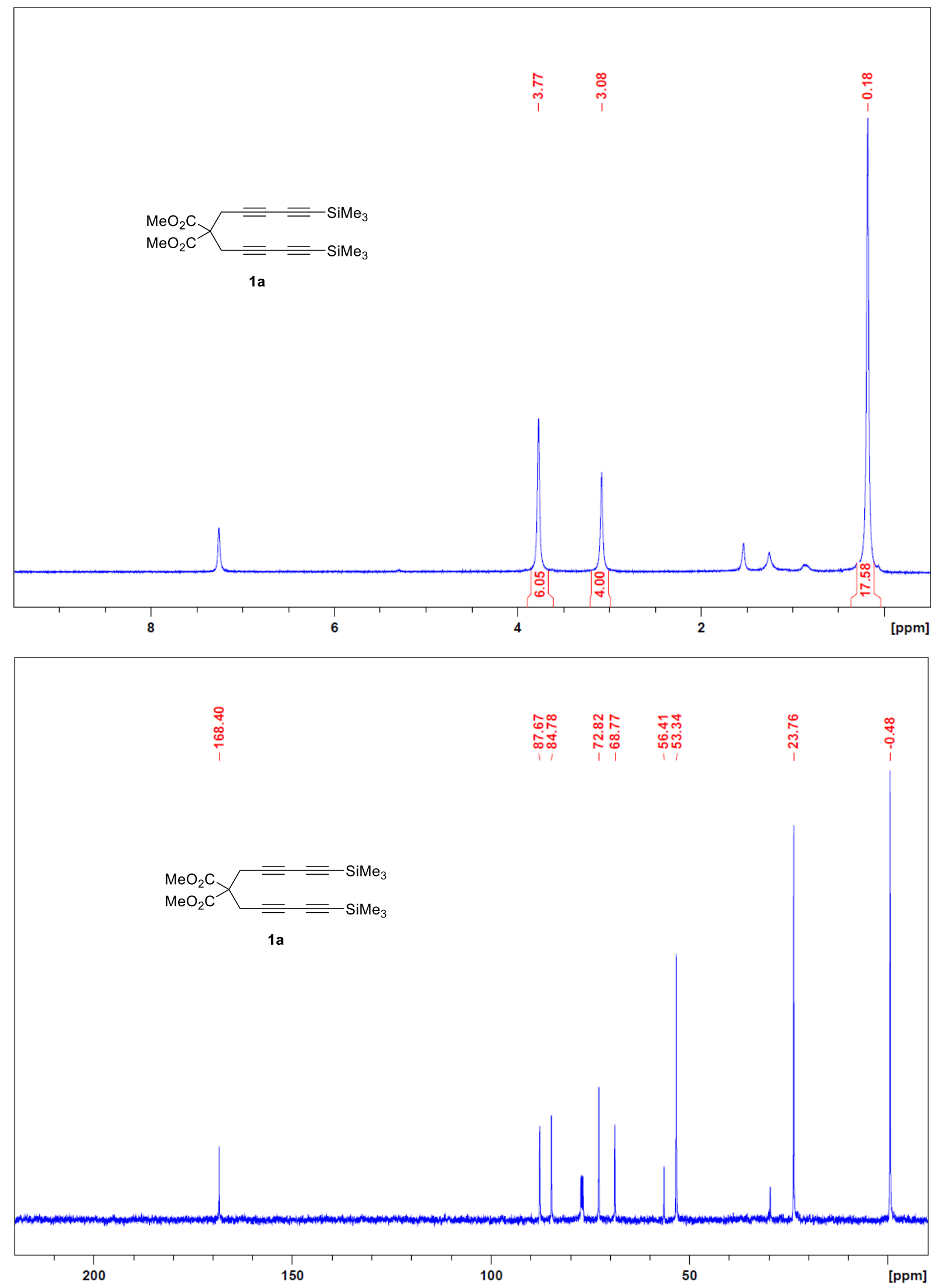


\section{กั}

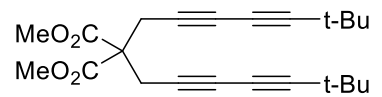

$1 b$
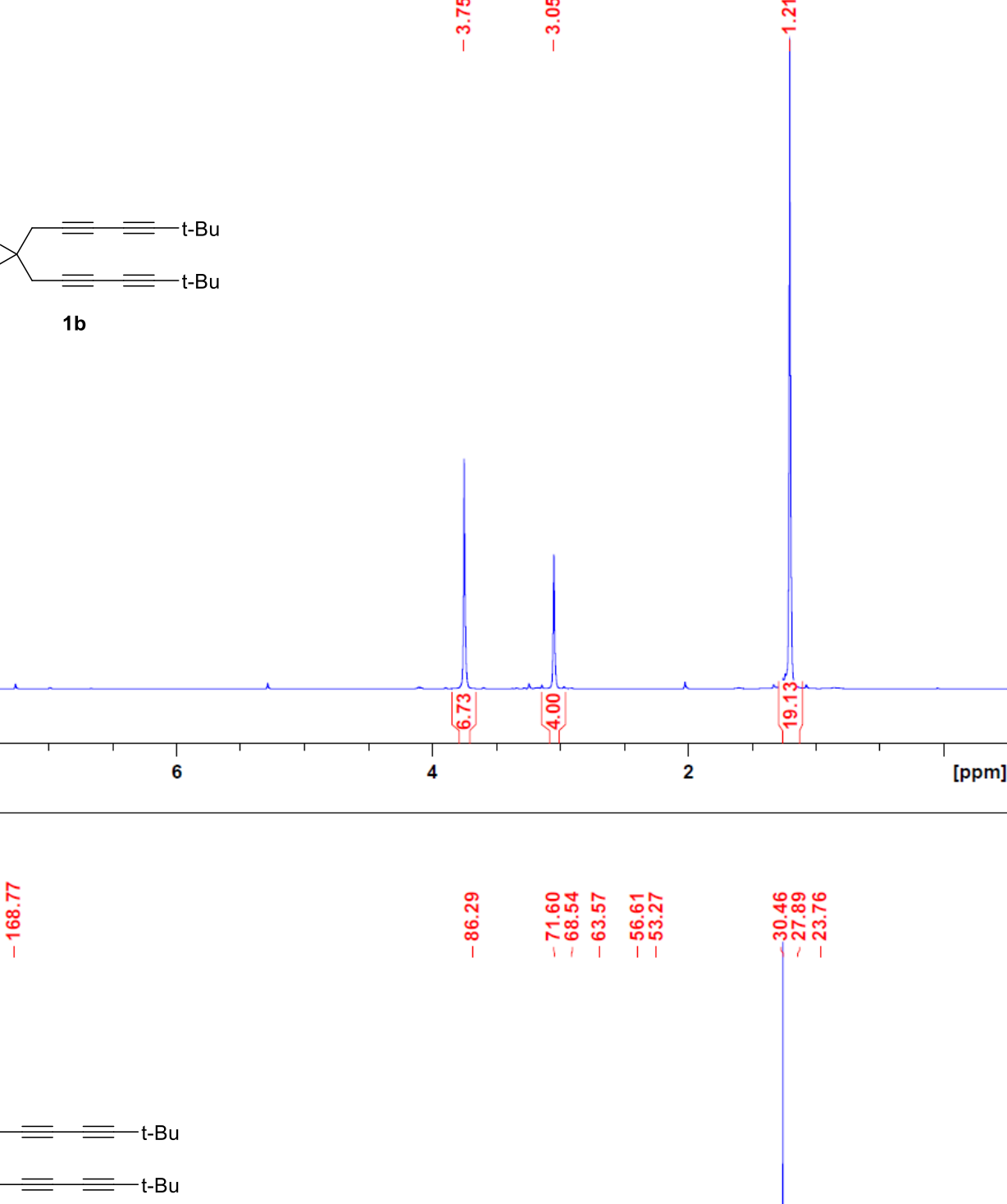

กุ ํํำ โกิ

ํำ

त०

อ्लกิ

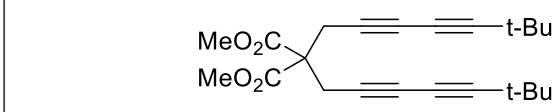

1b
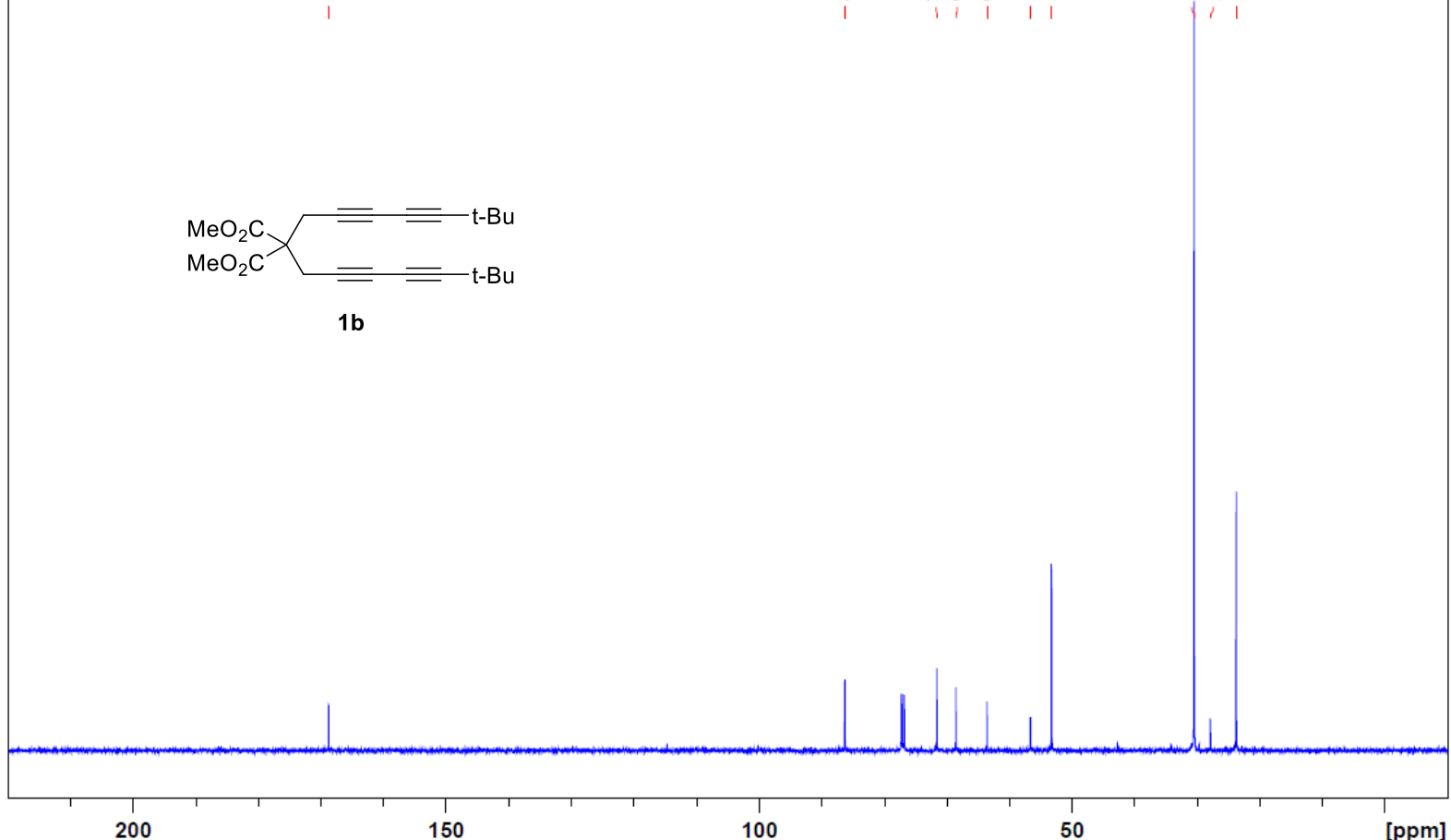

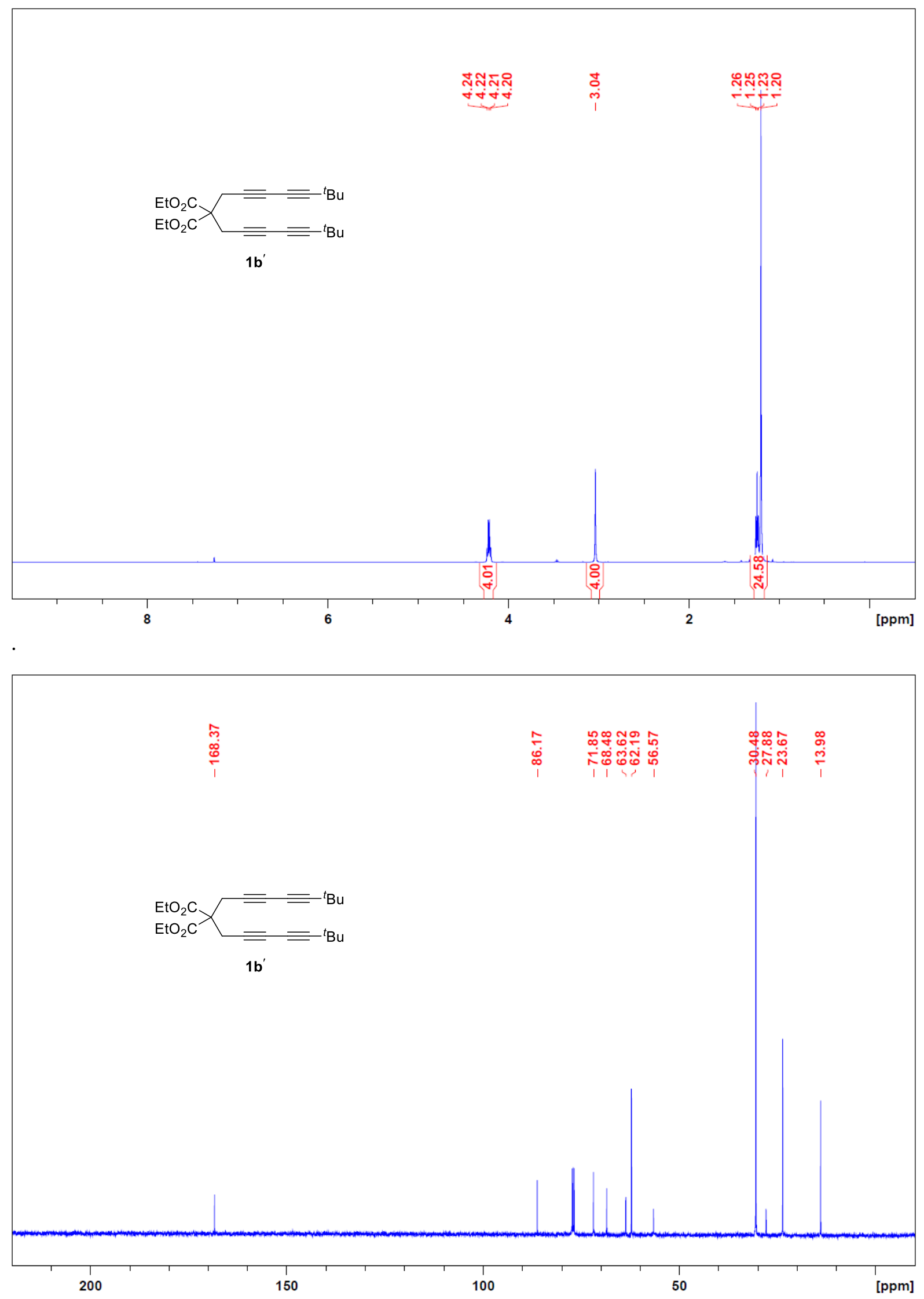

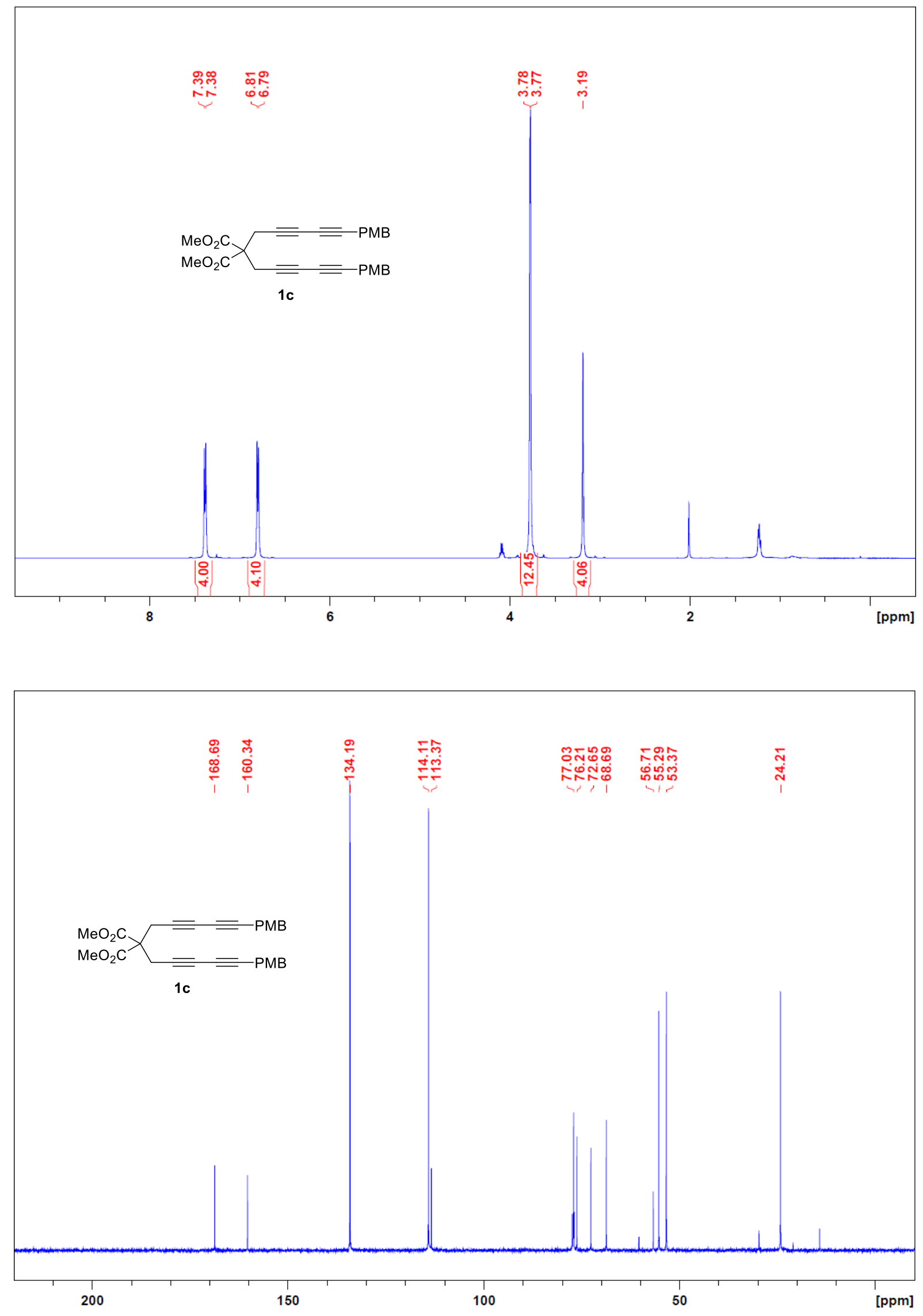

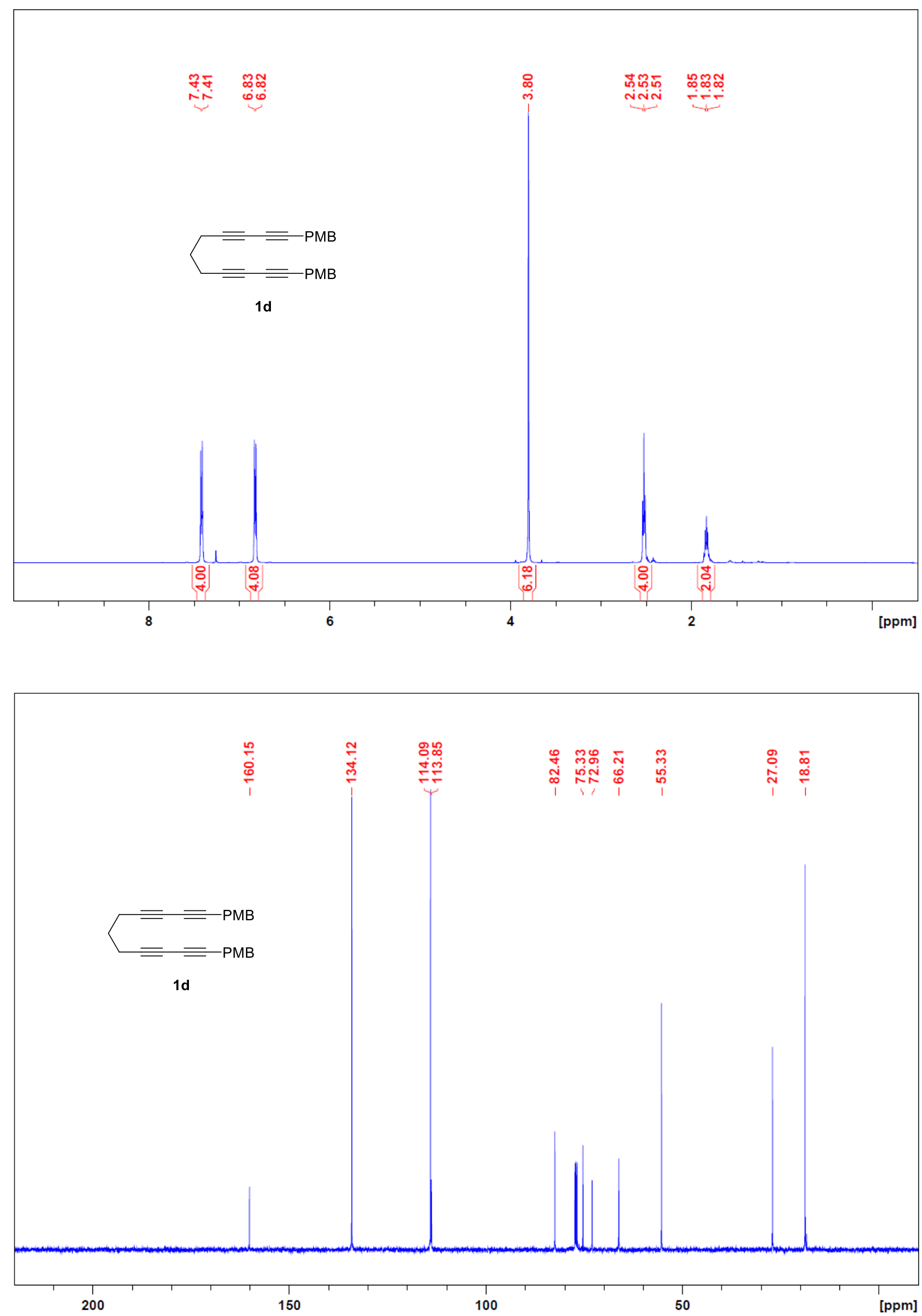

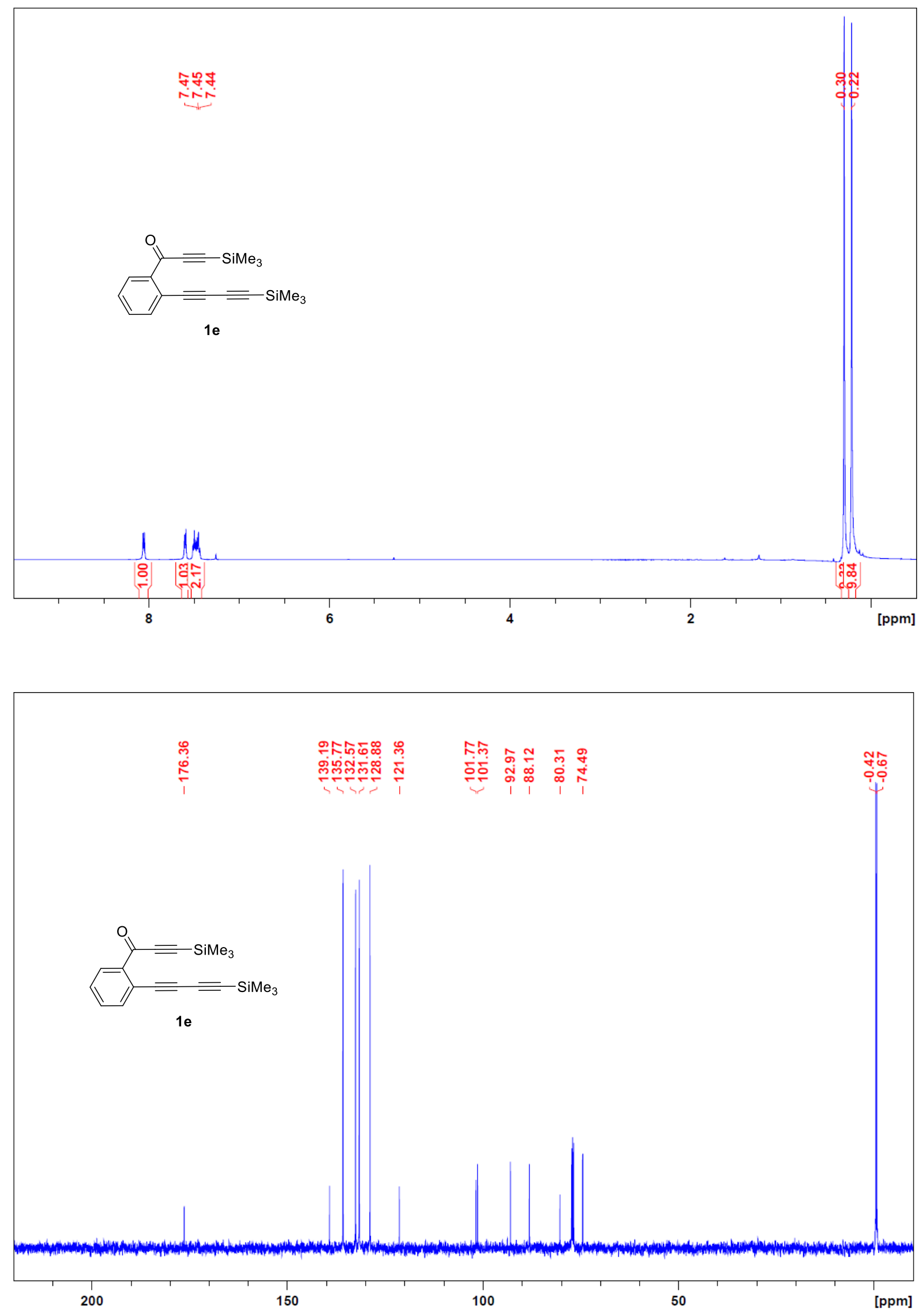

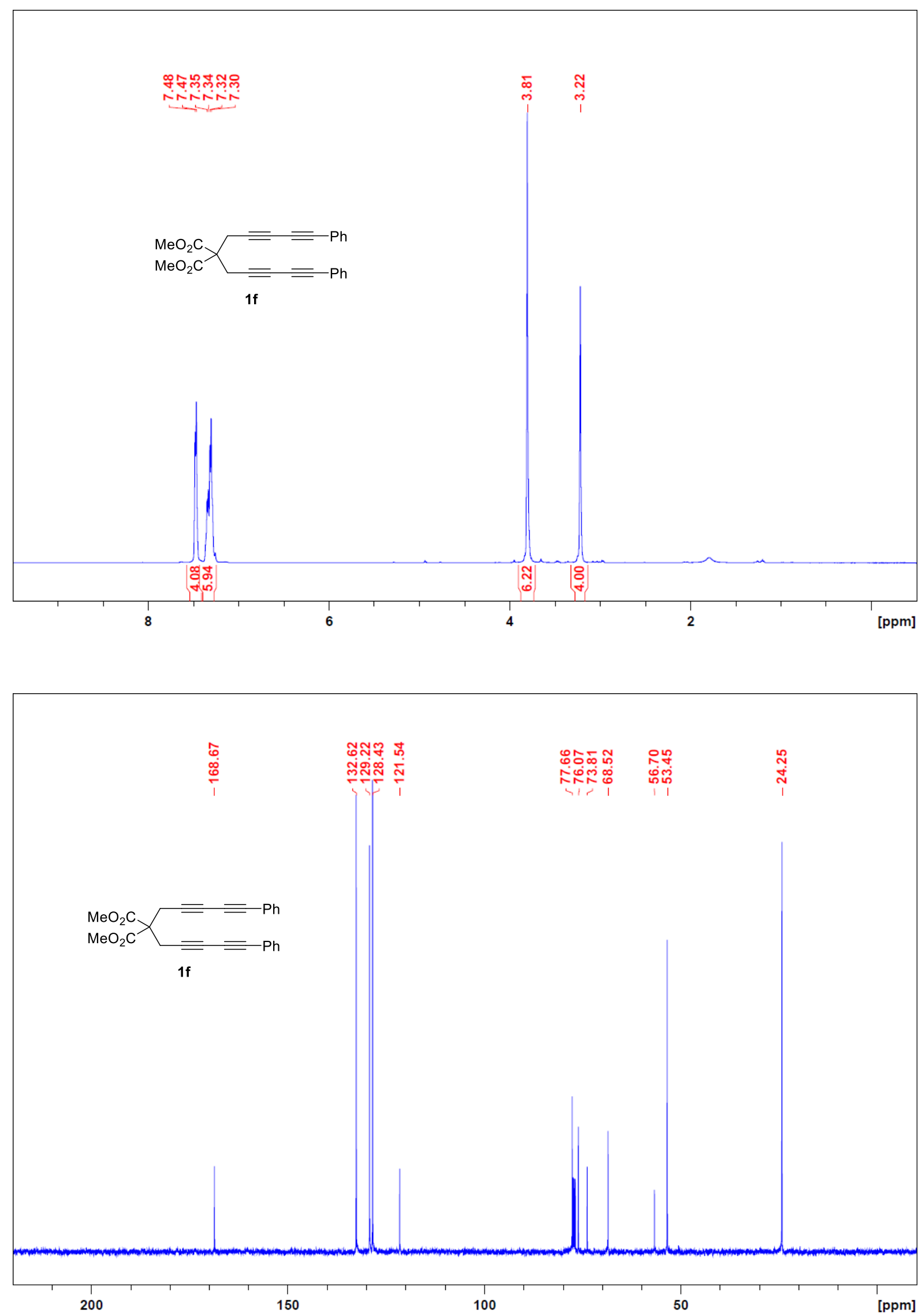

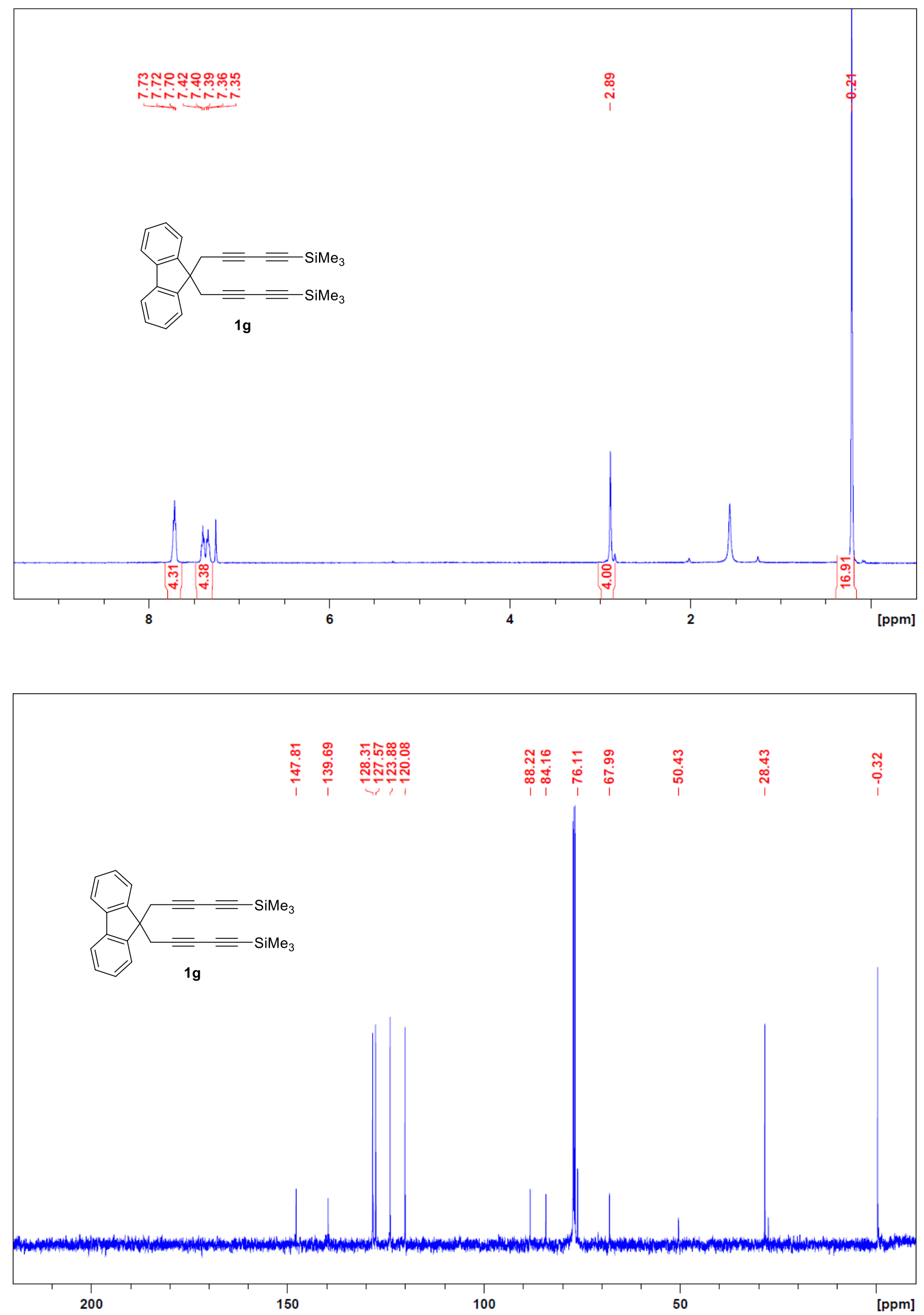


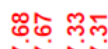

NN

$\stackrel{\infty}{\stackrel{\infty}{+}}$

พับ

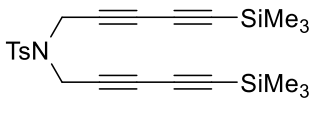

1h
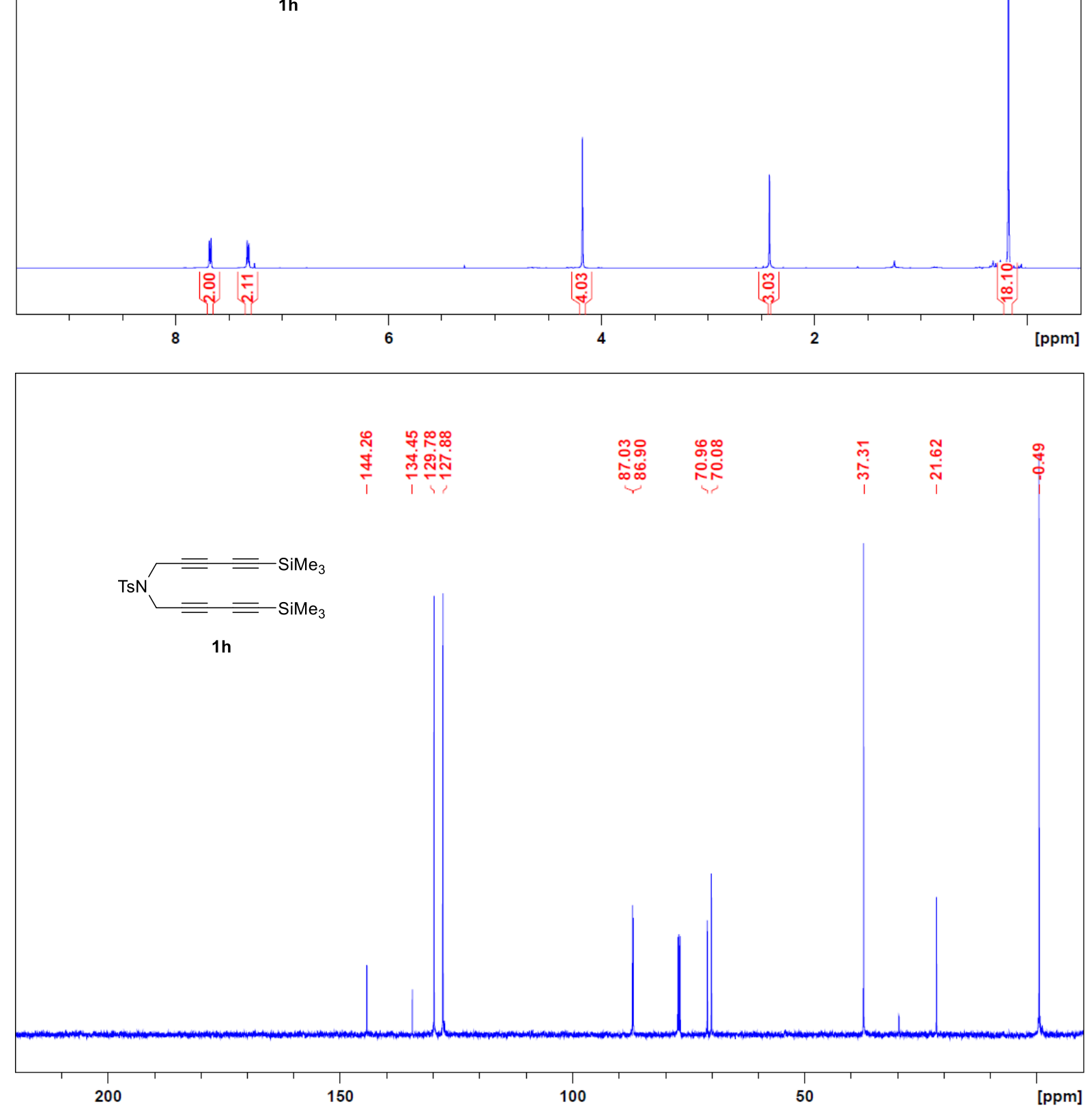

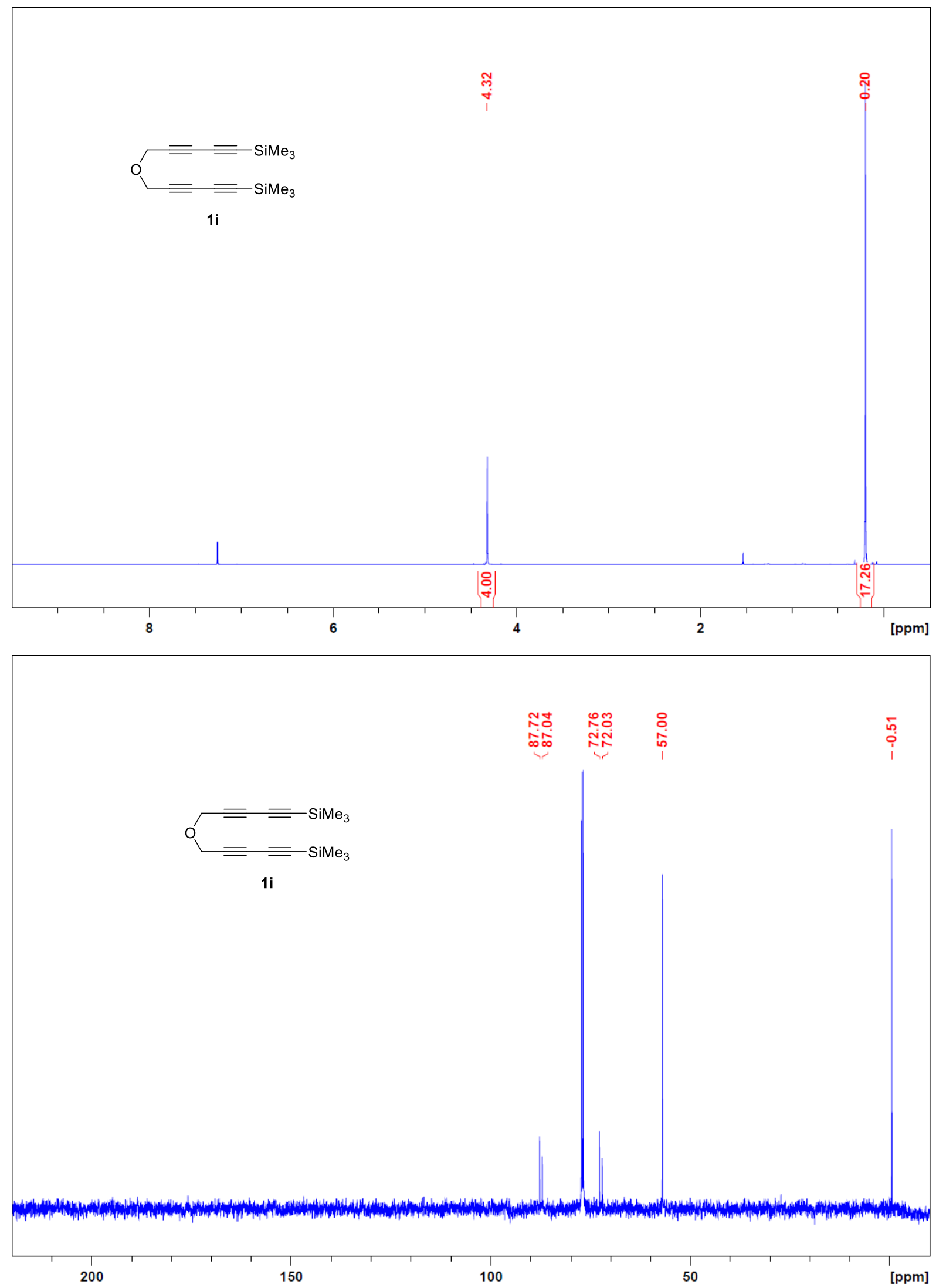

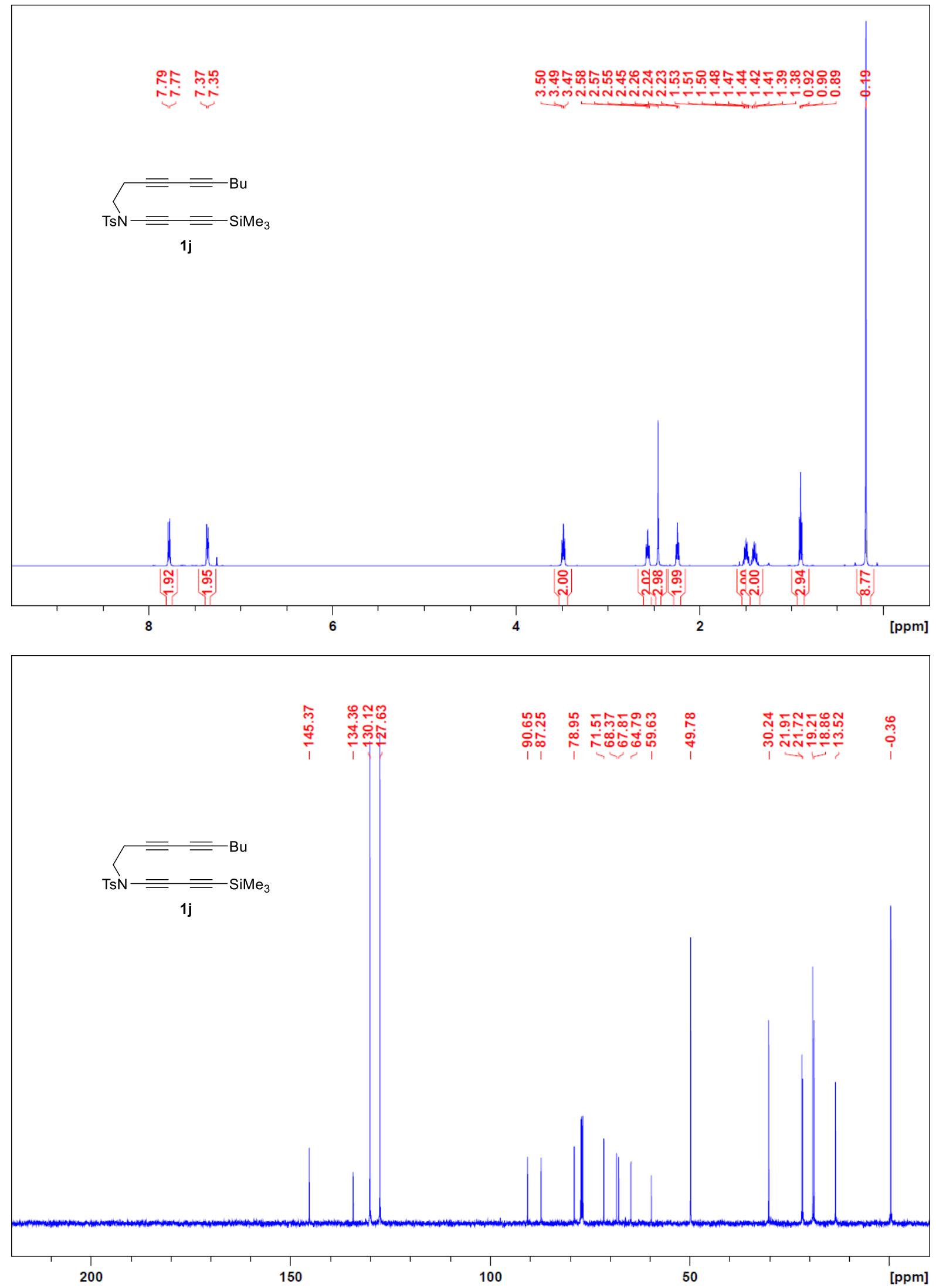

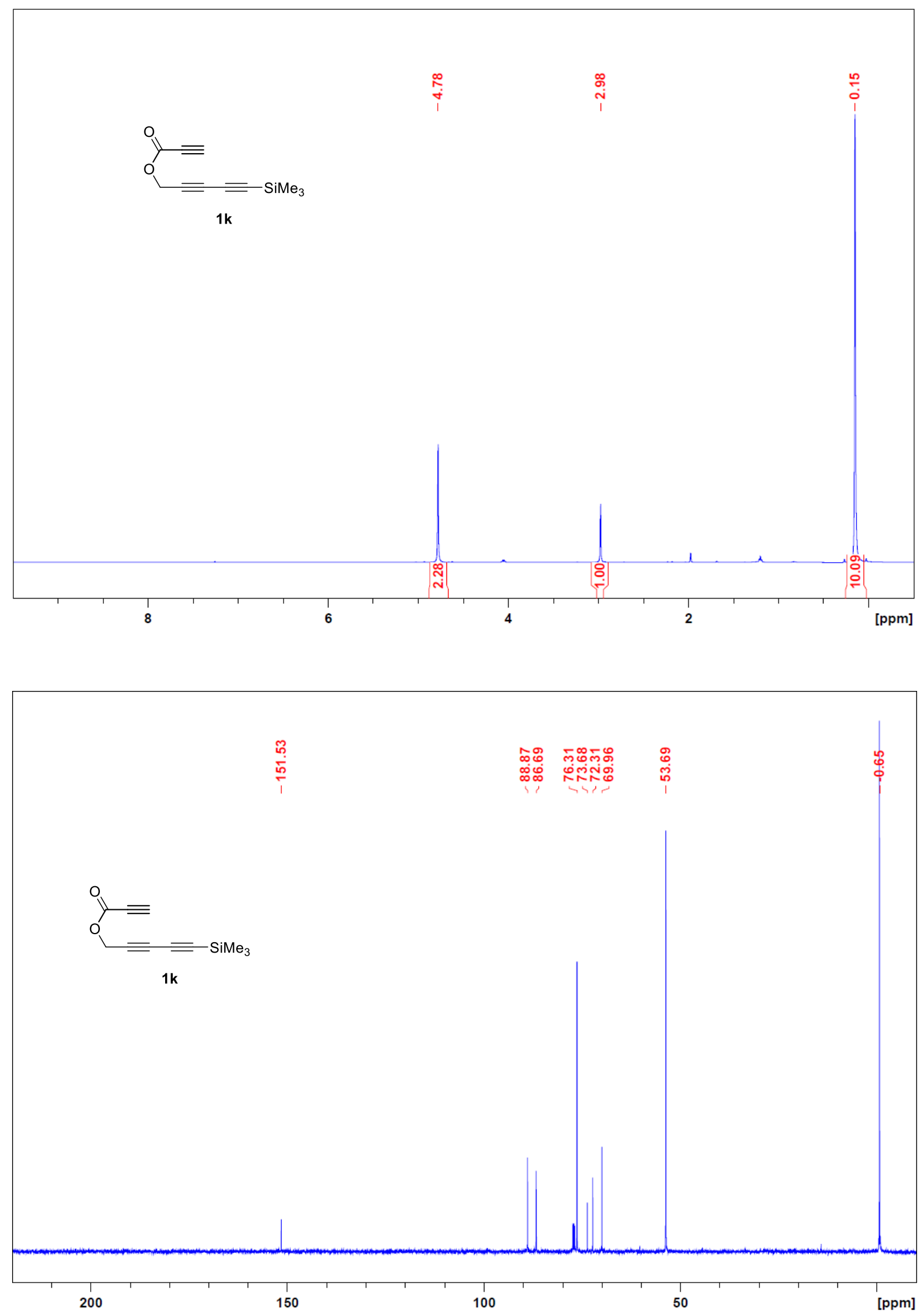

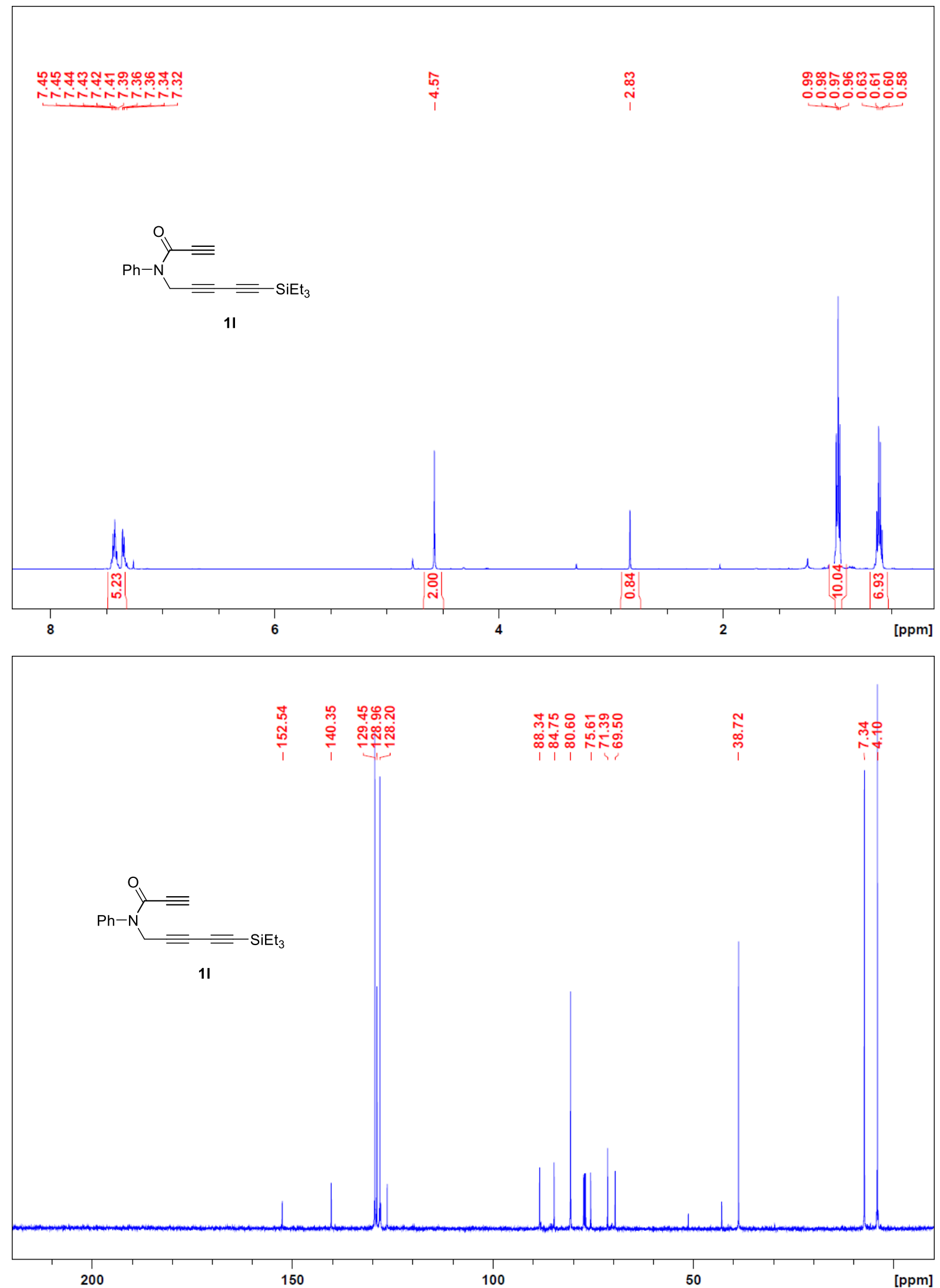

NMR Spectra of Quinazoline Products 

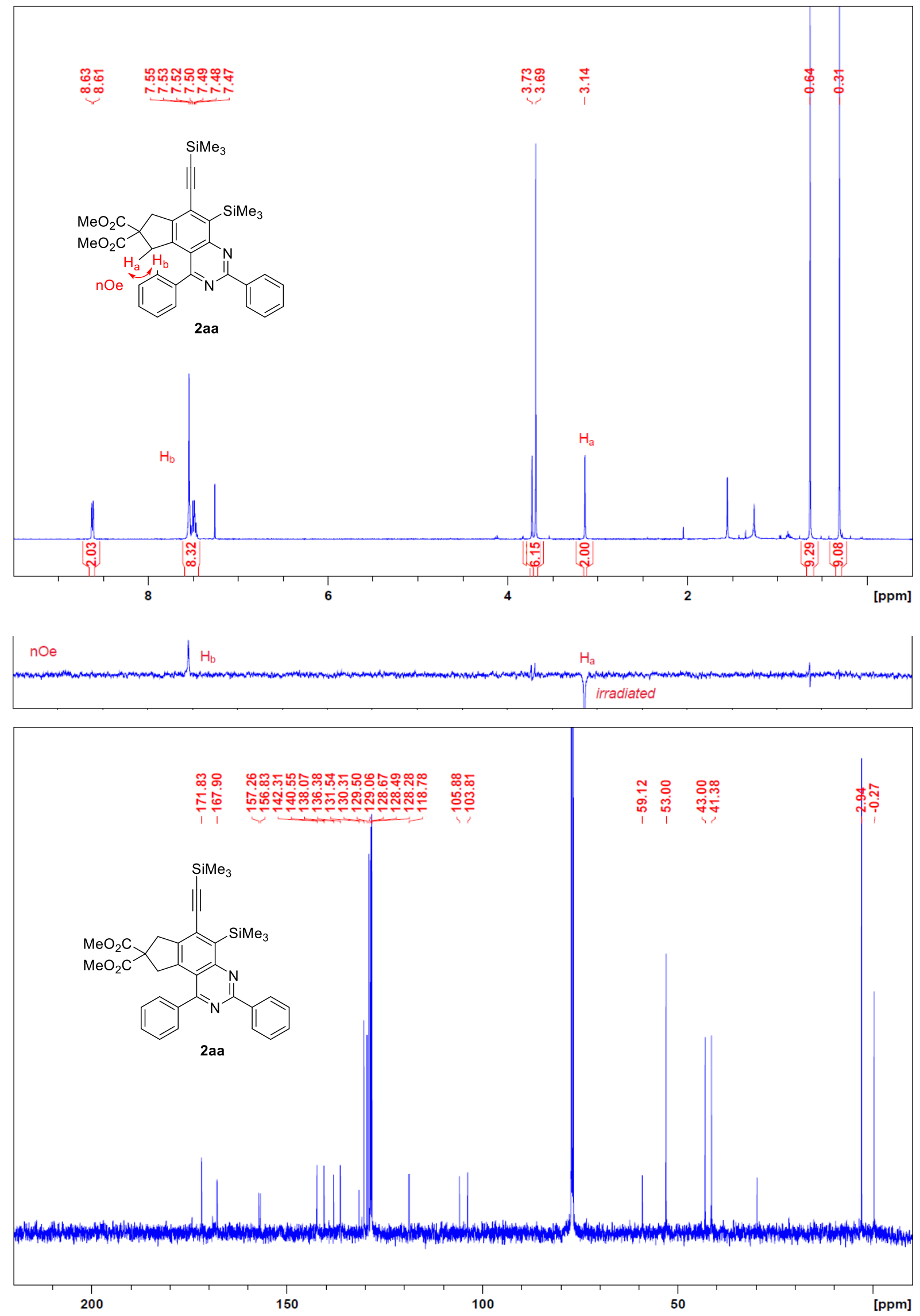

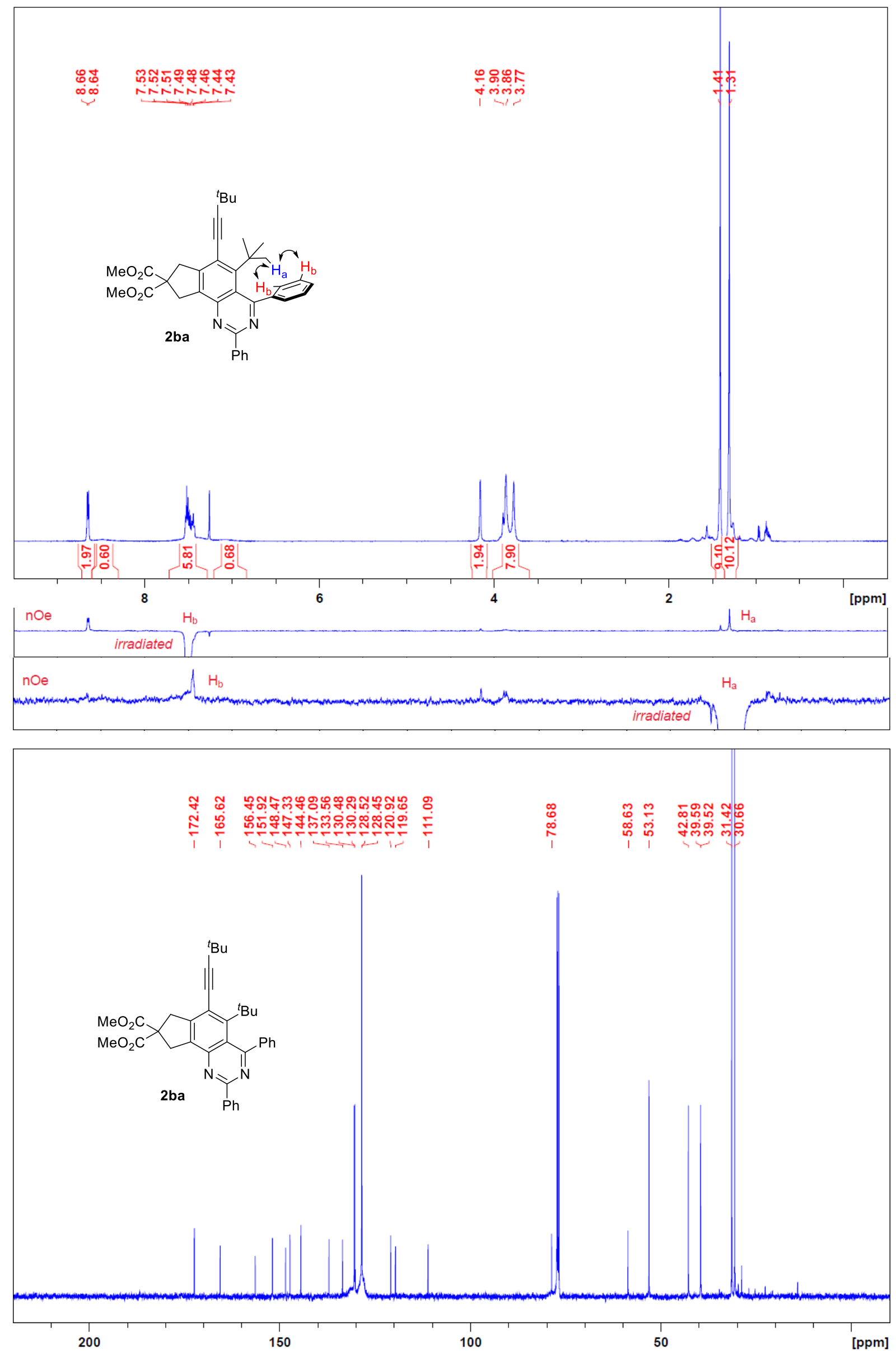

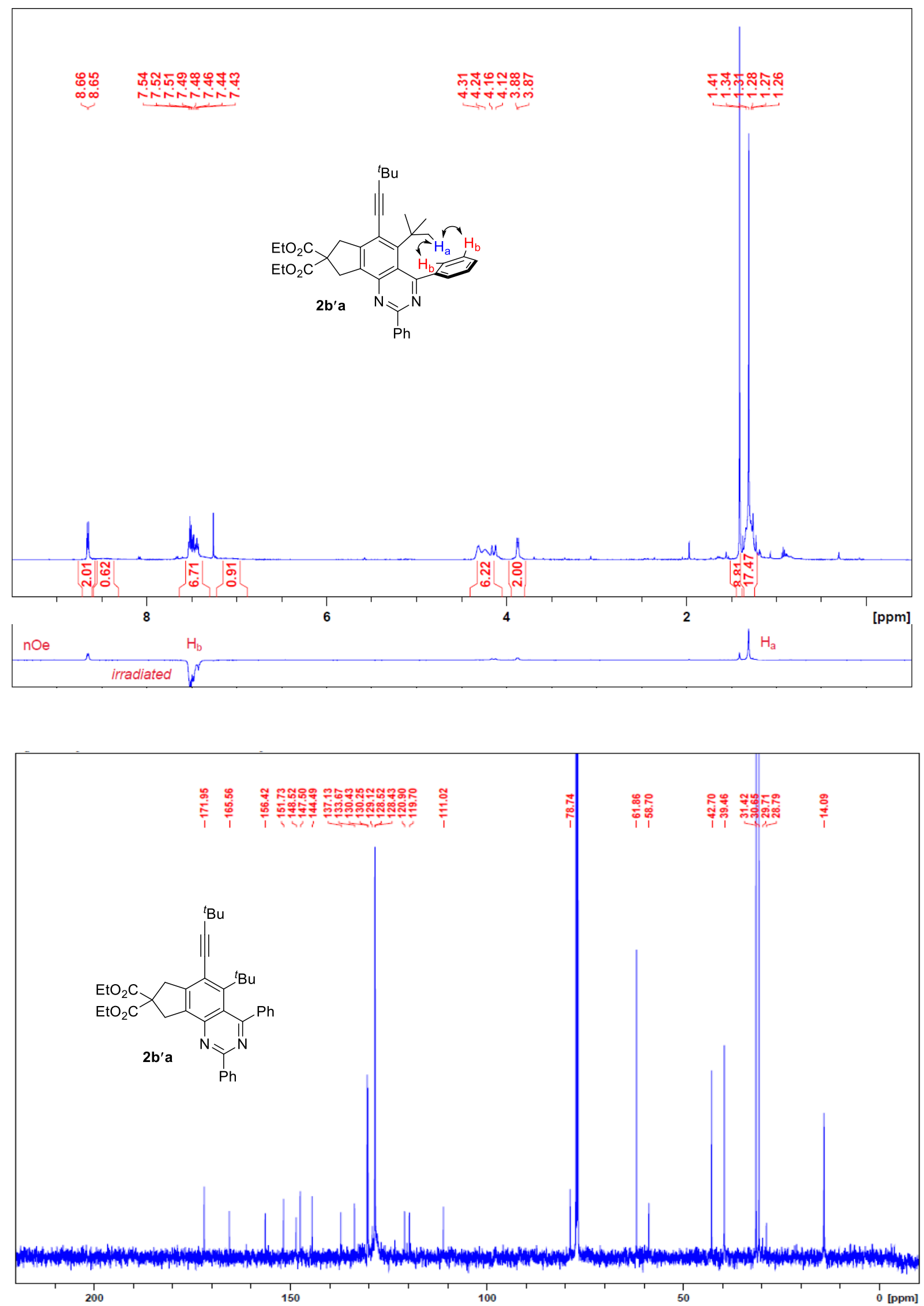

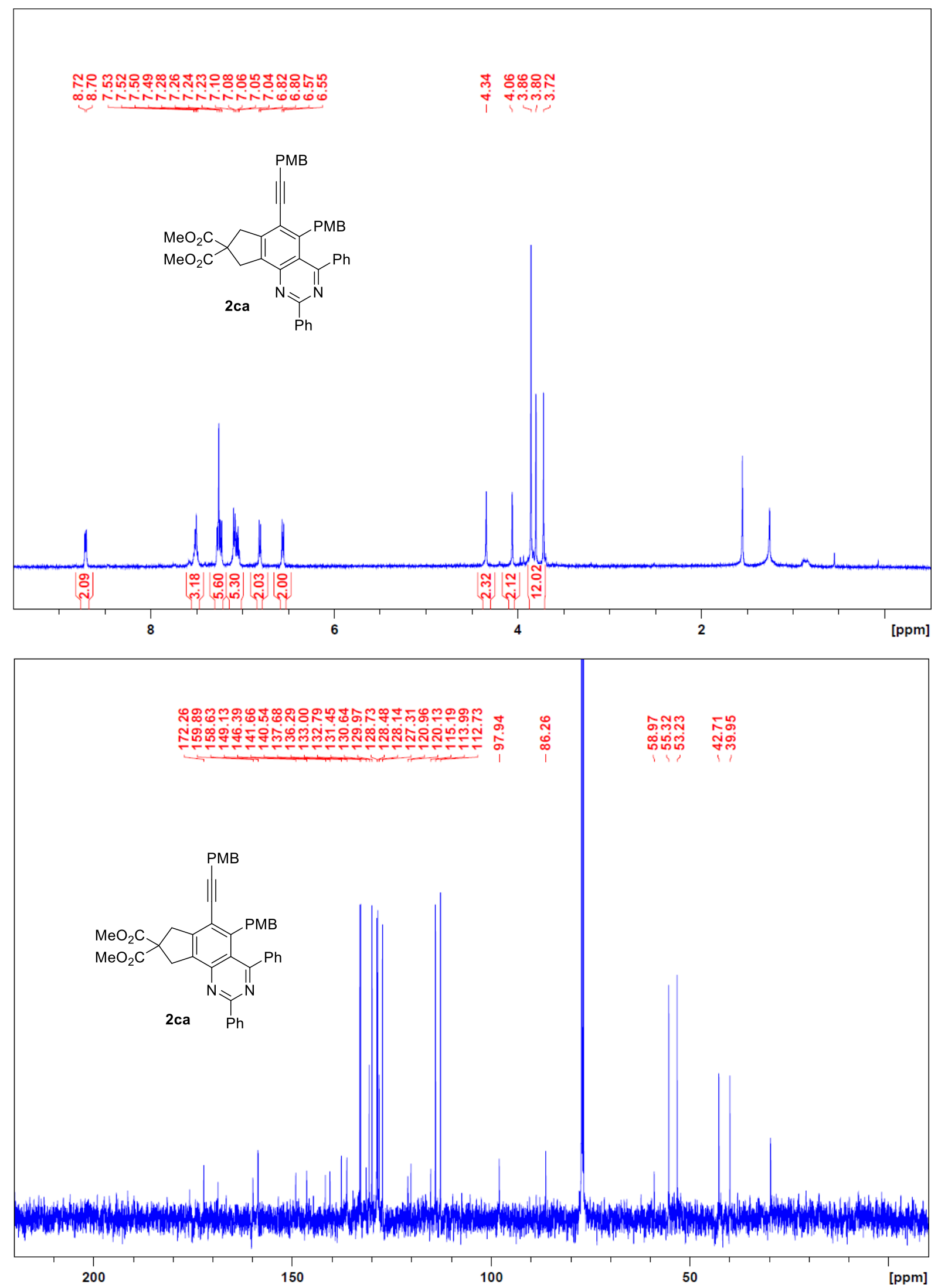


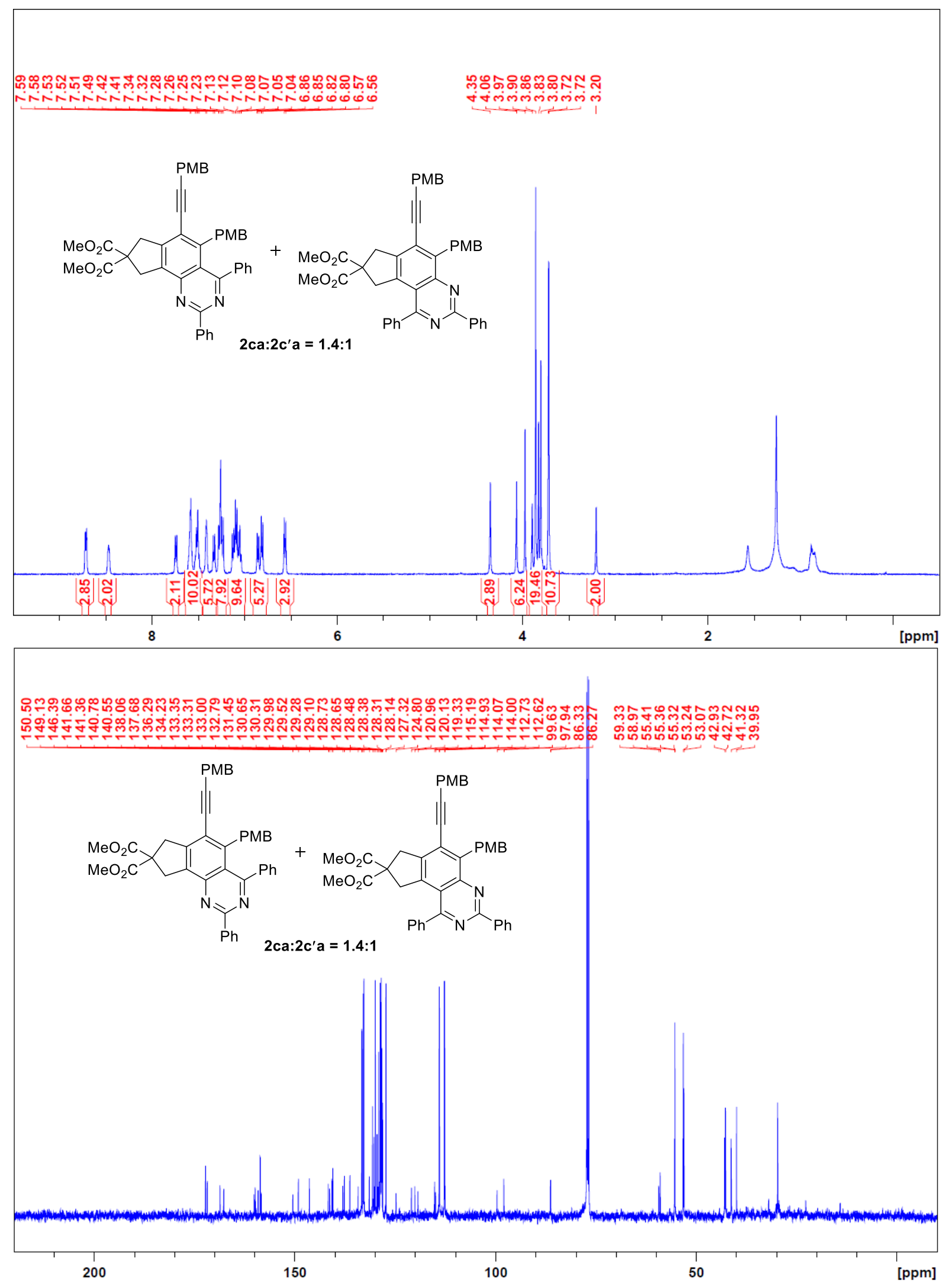



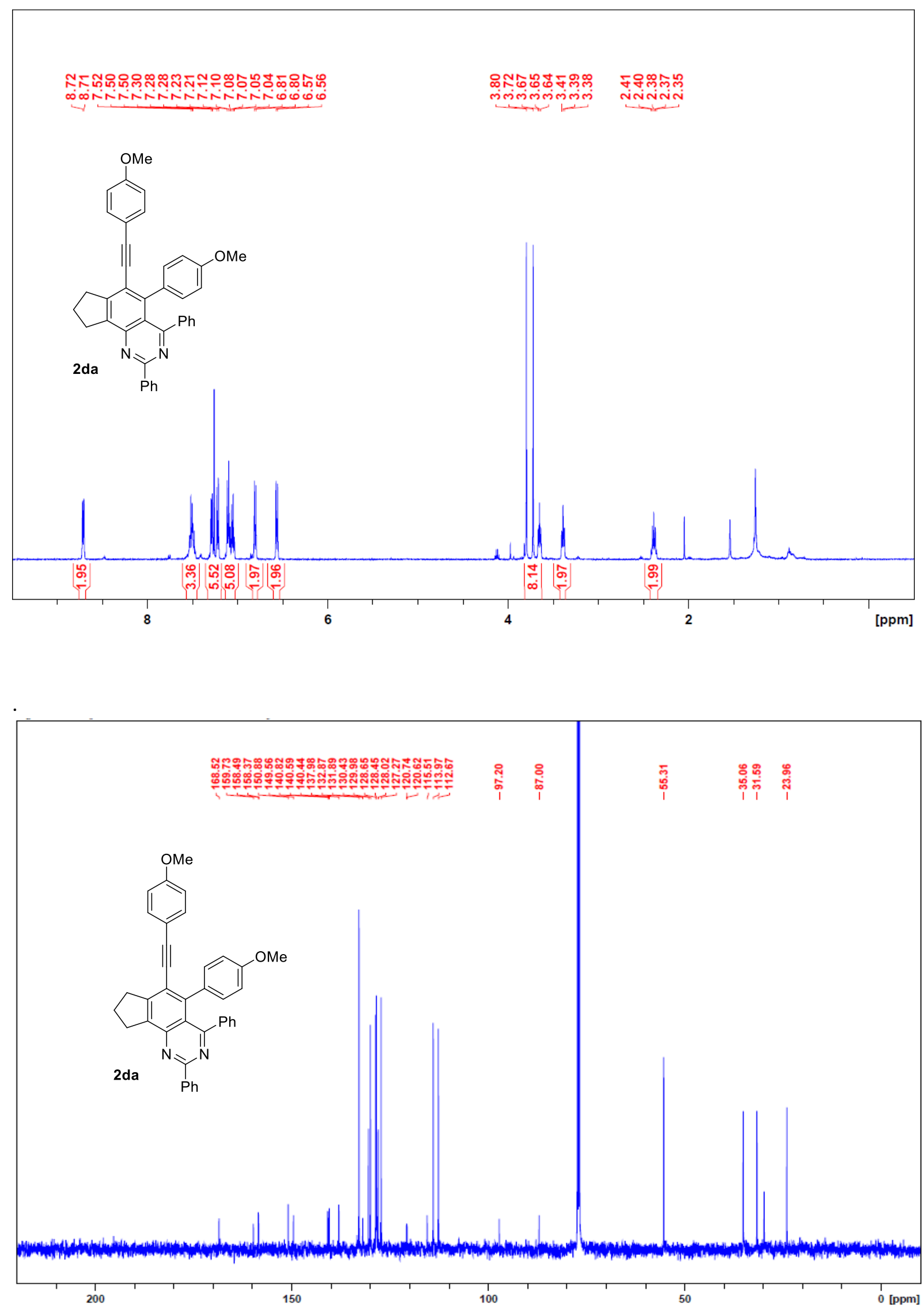

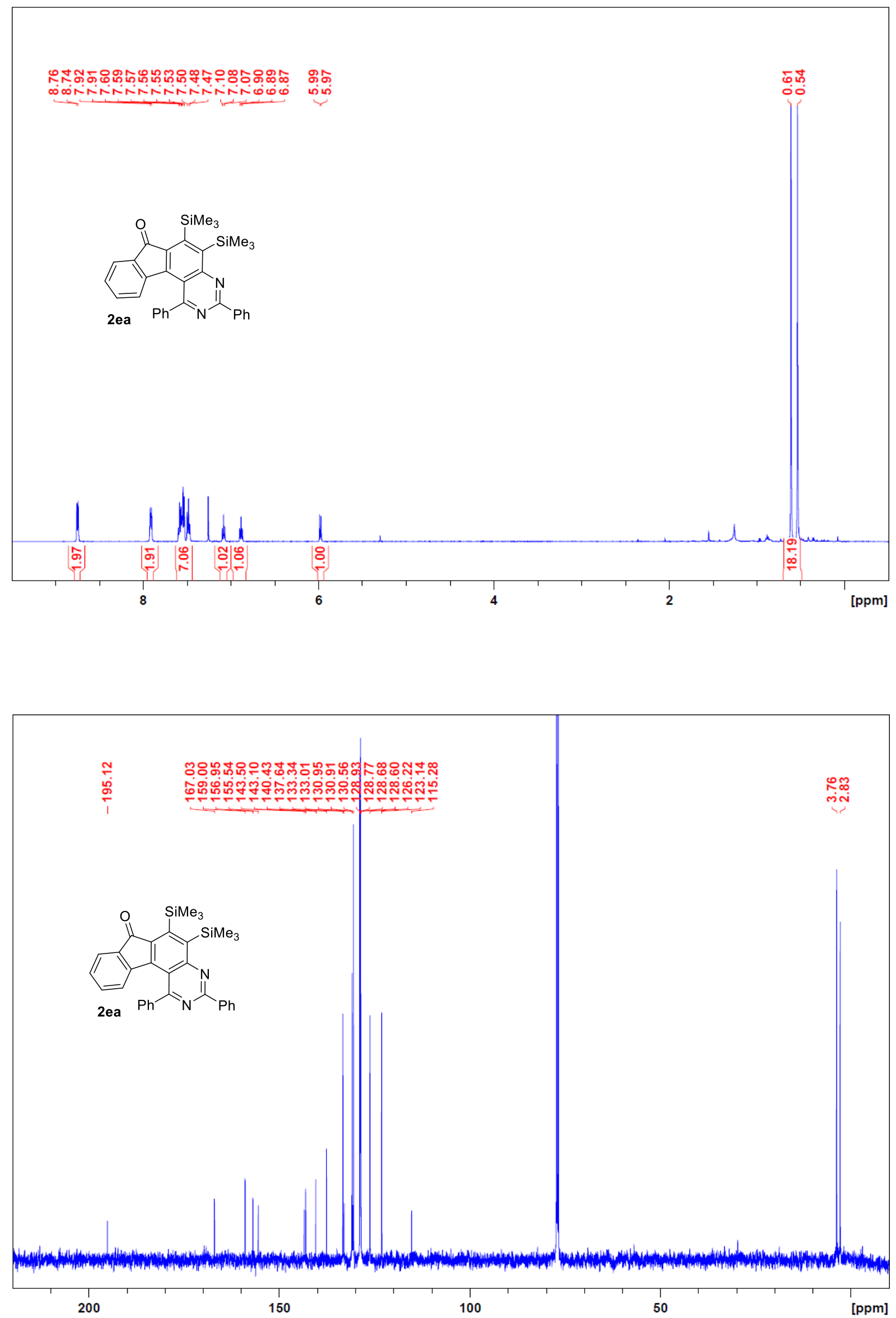

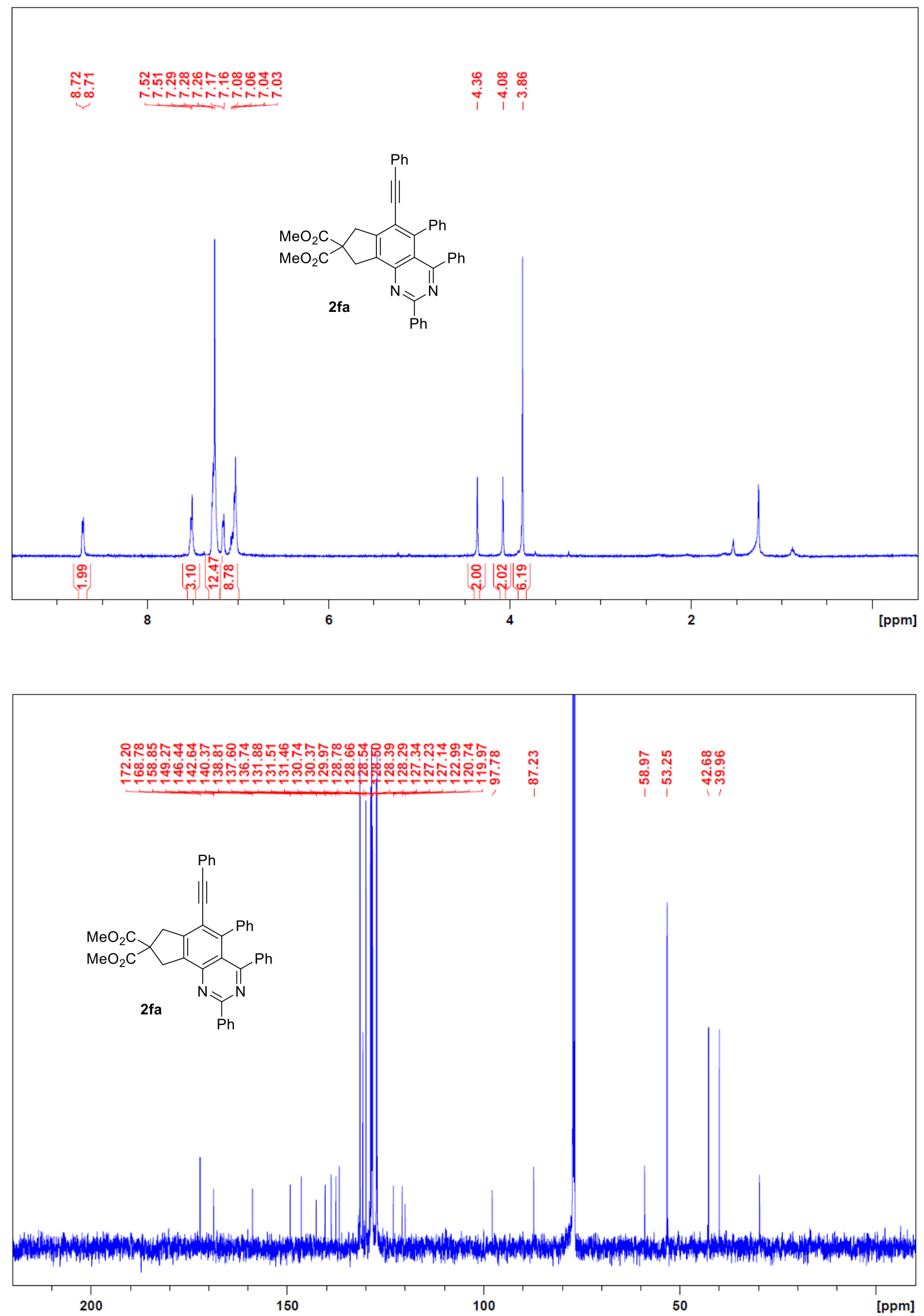

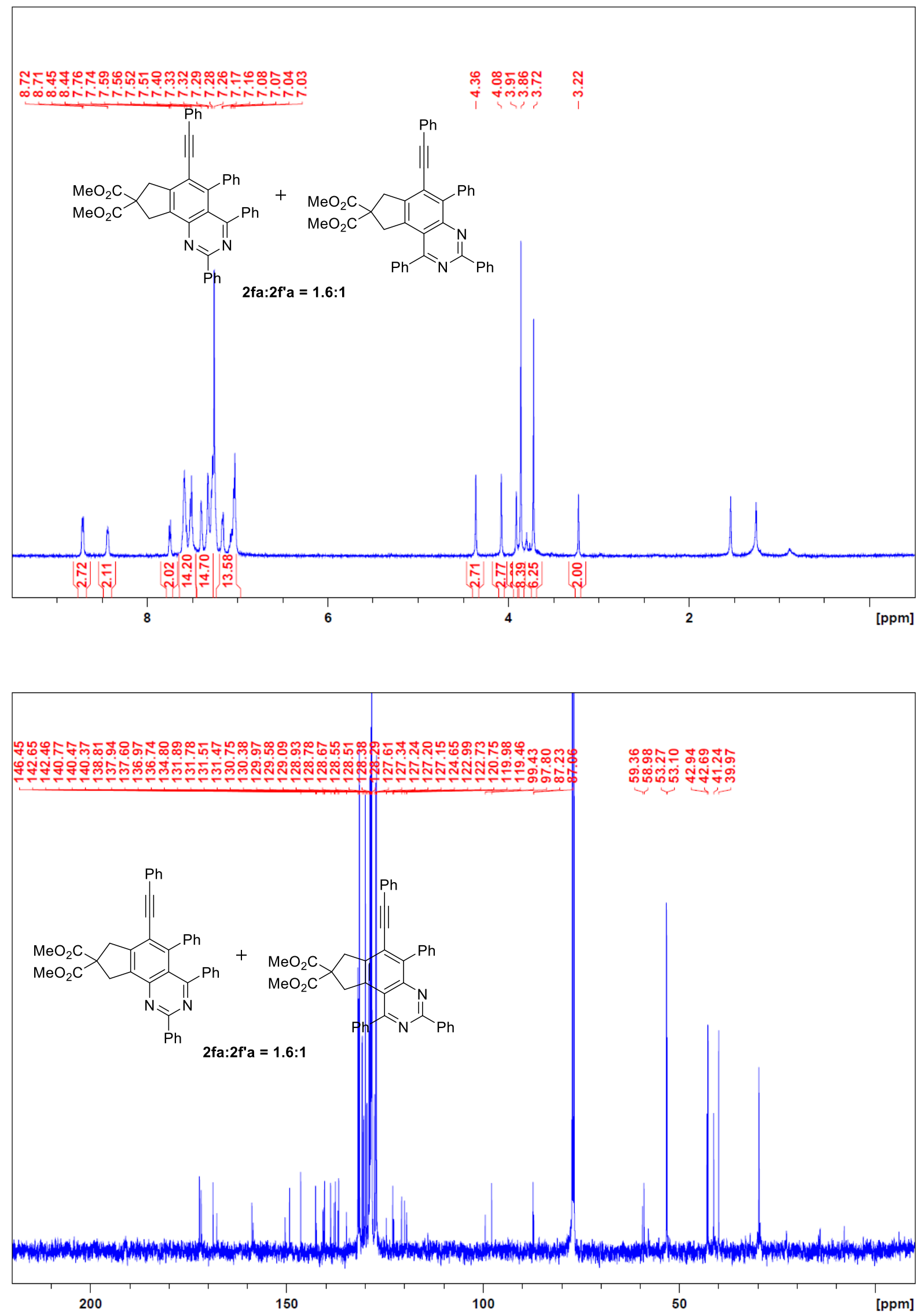

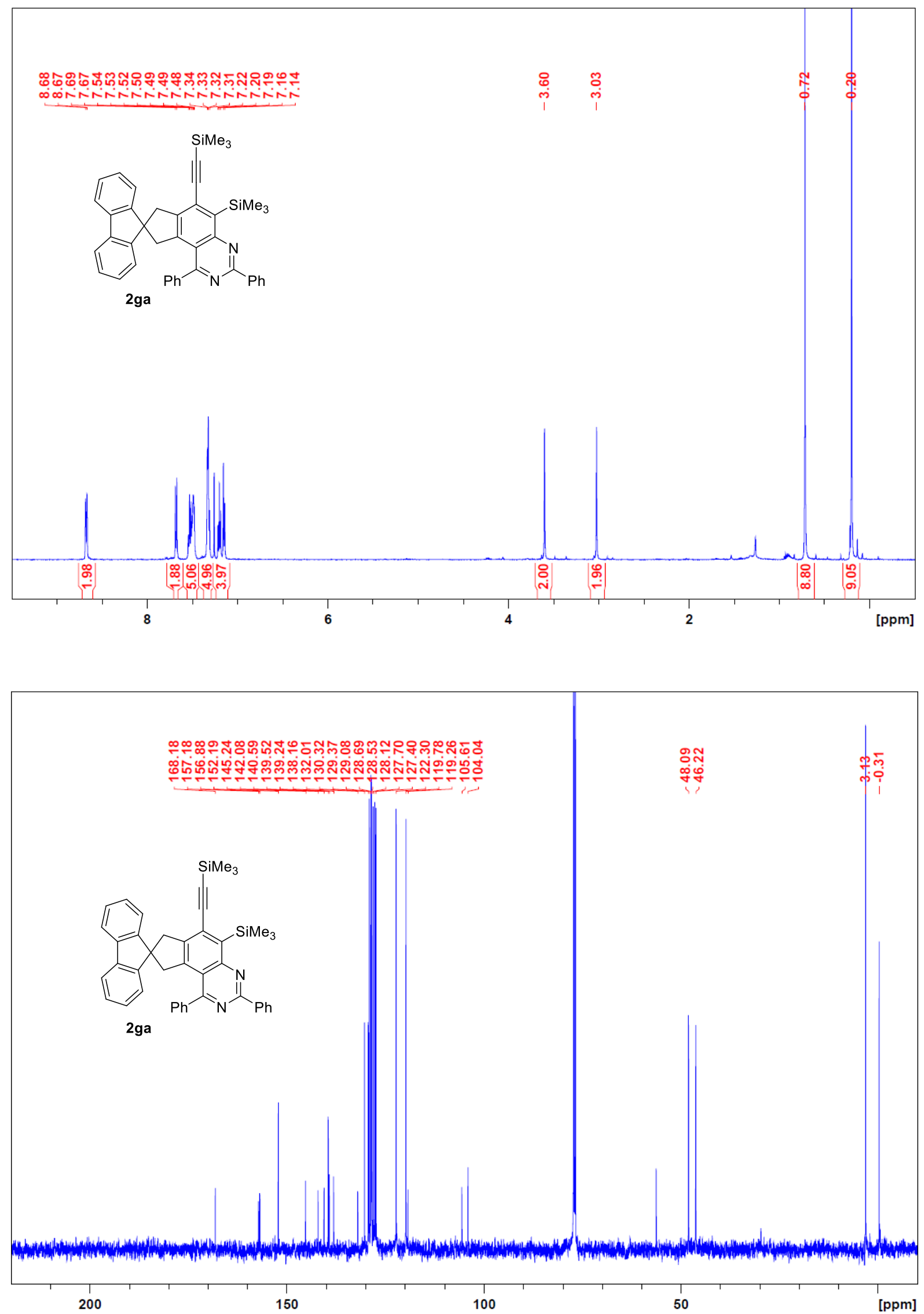

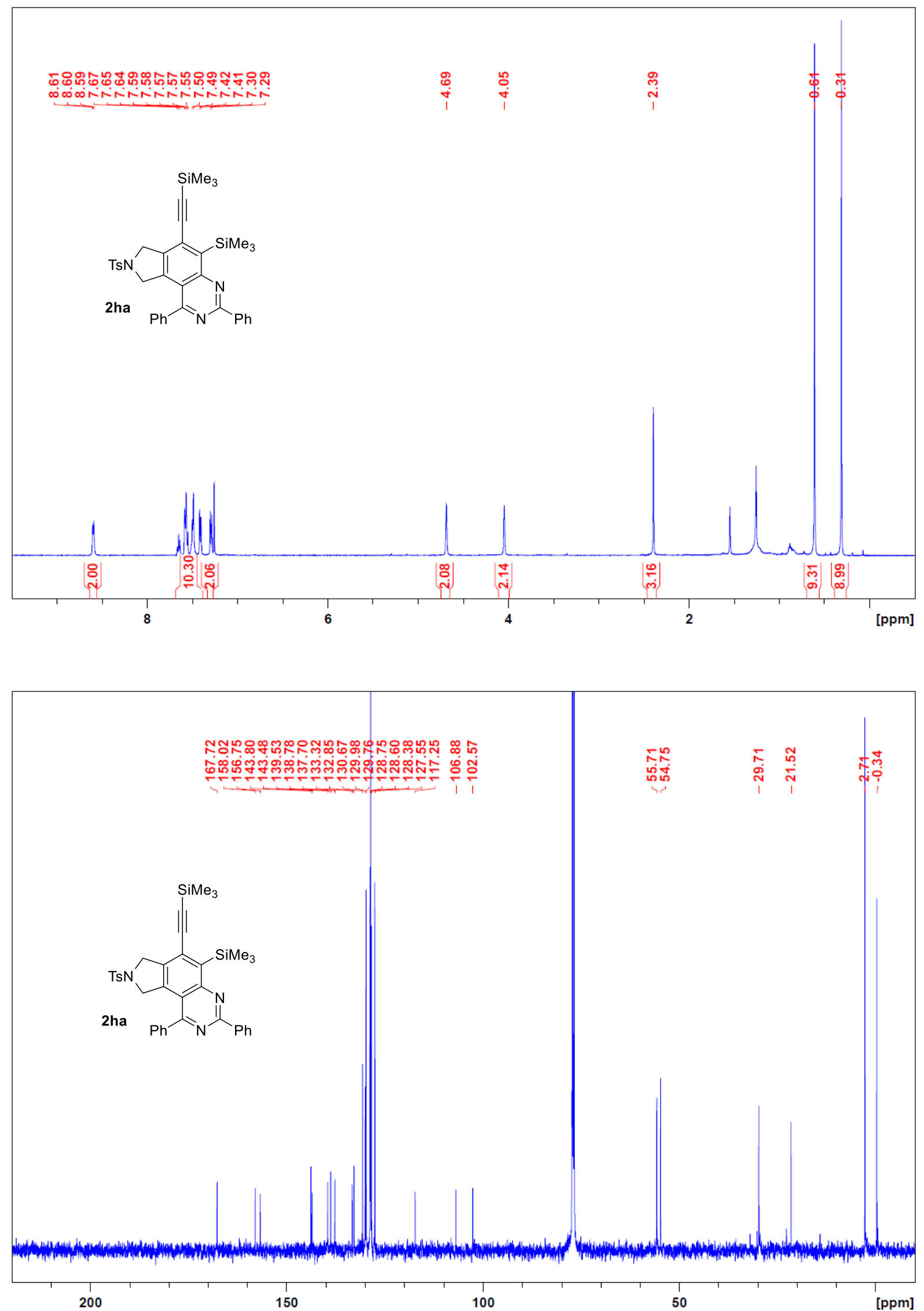

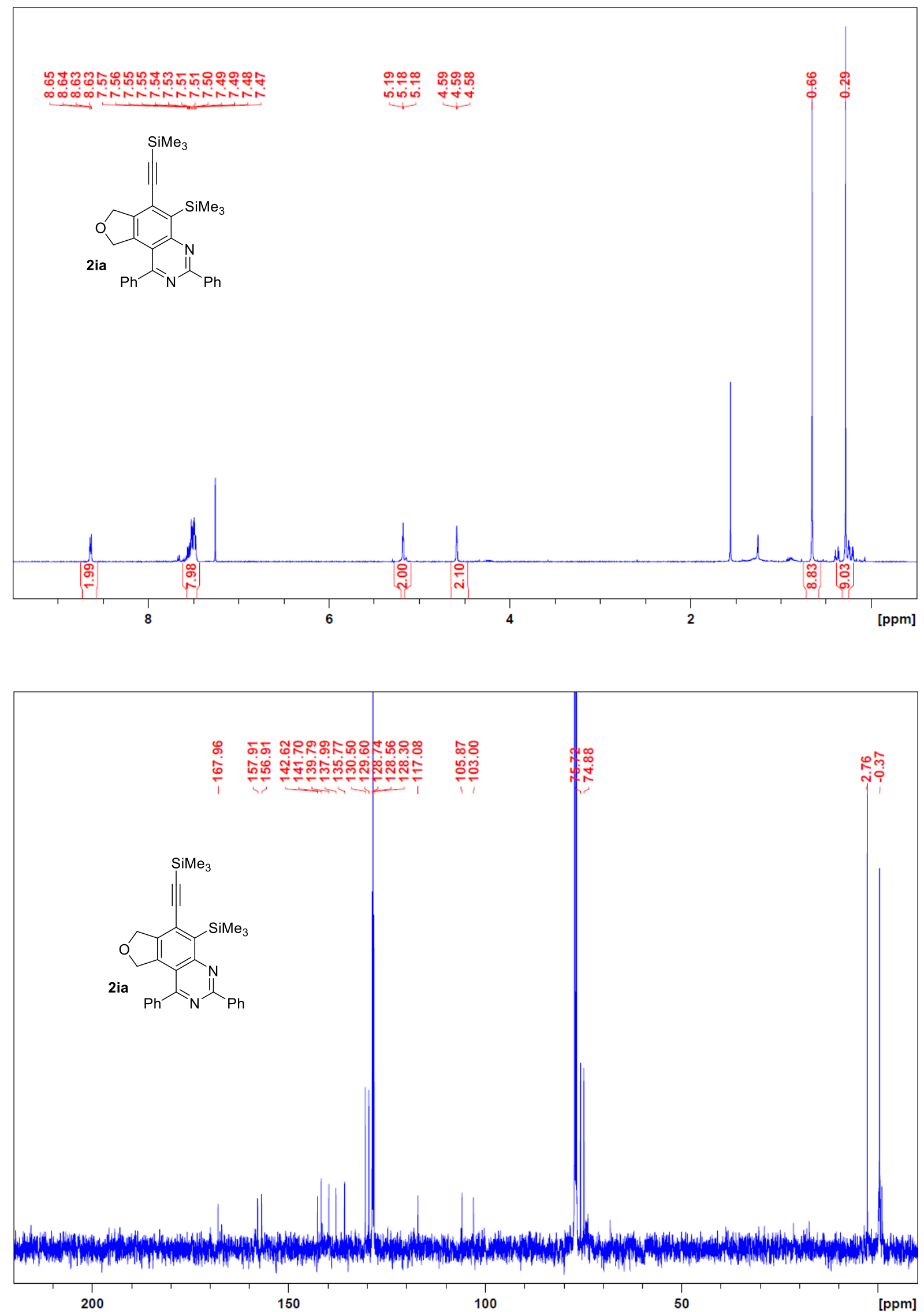

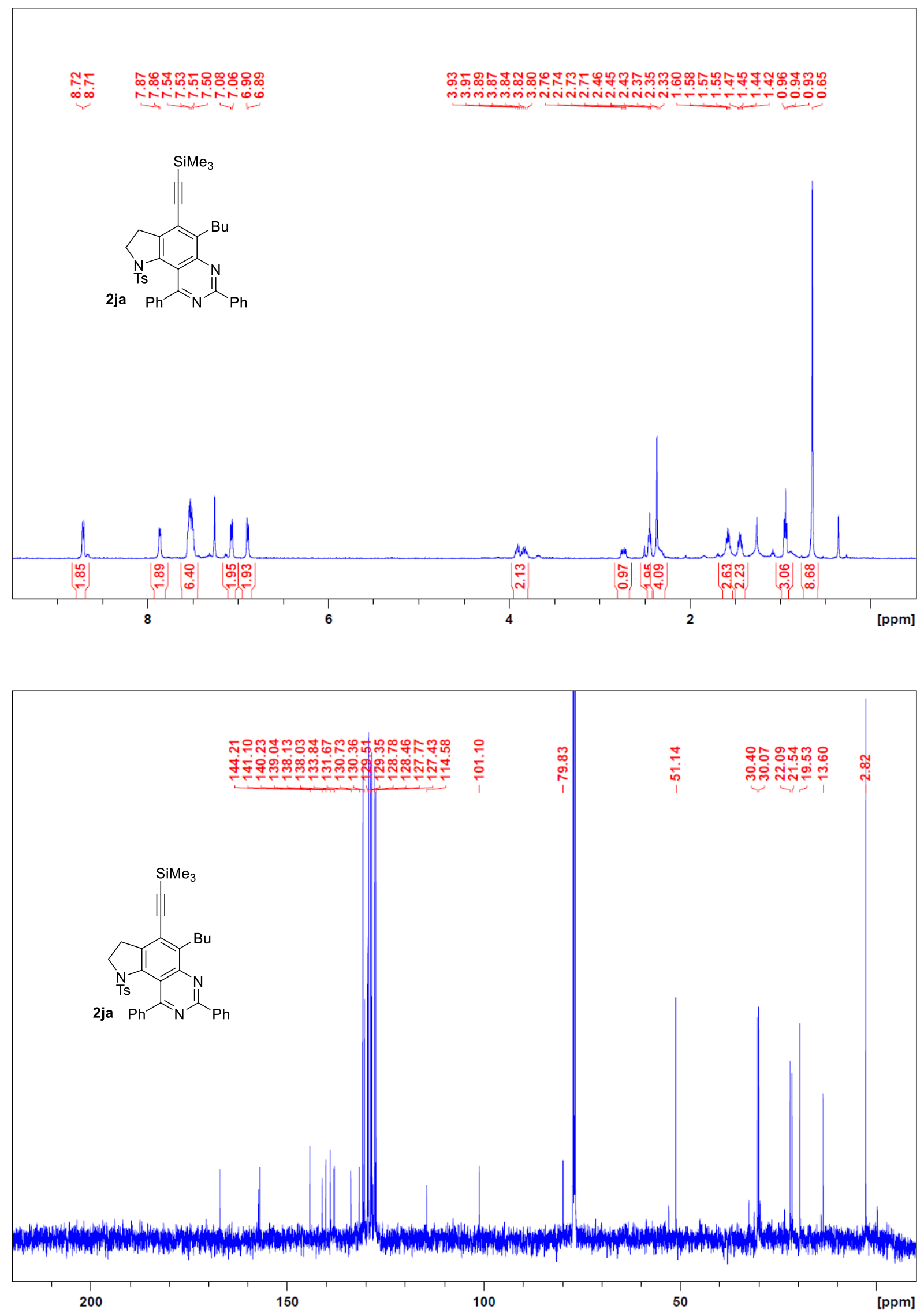

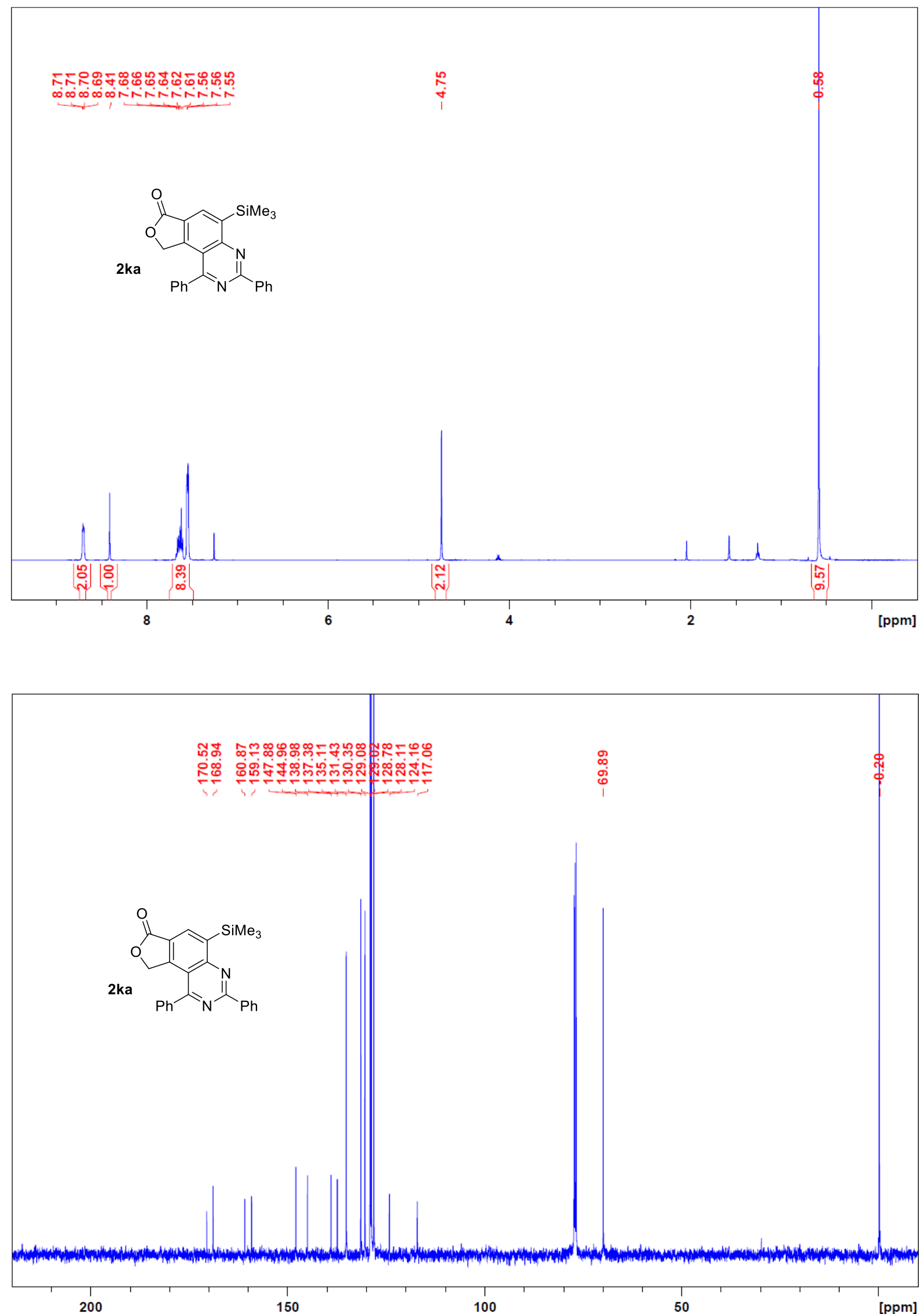

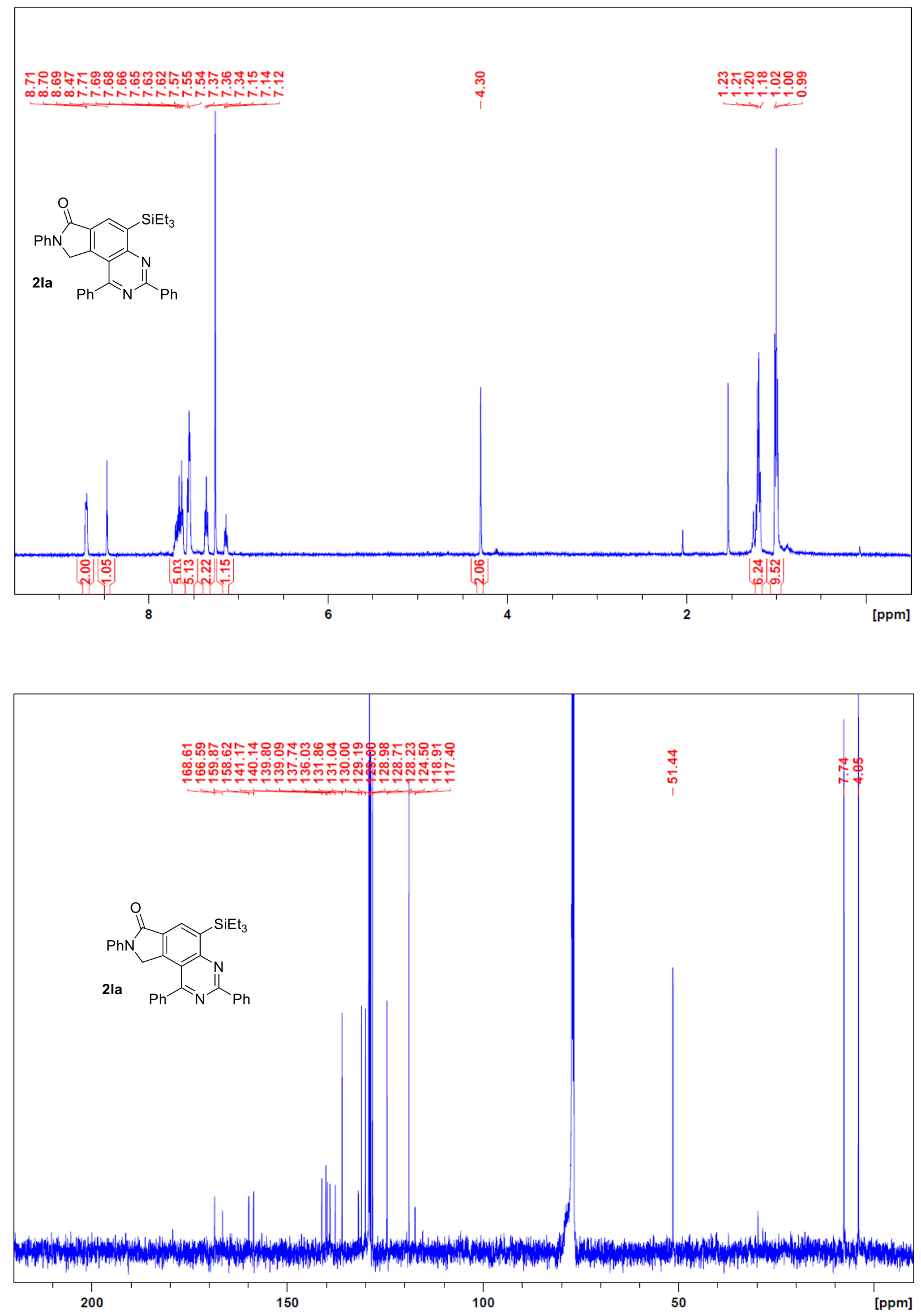


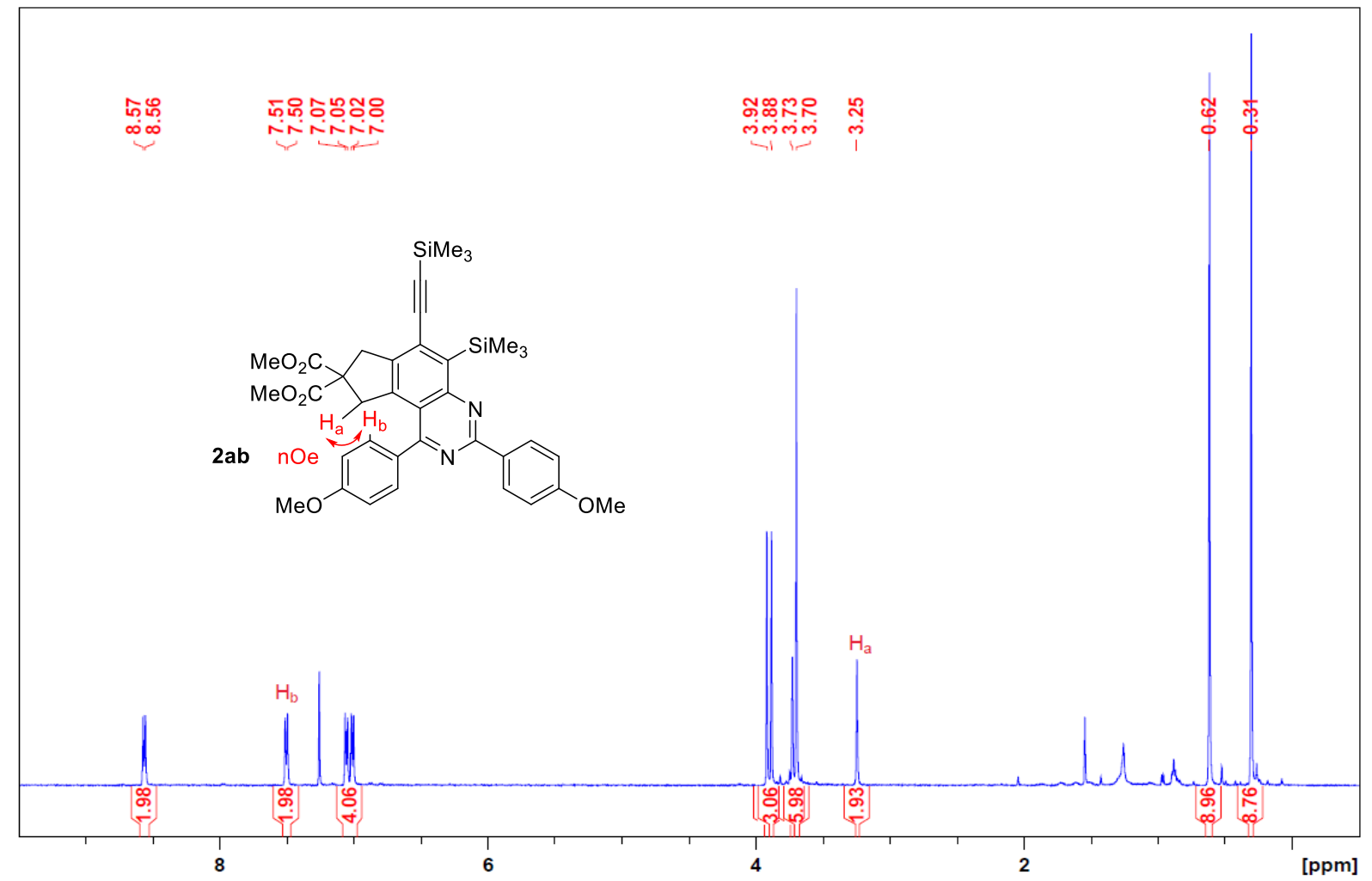

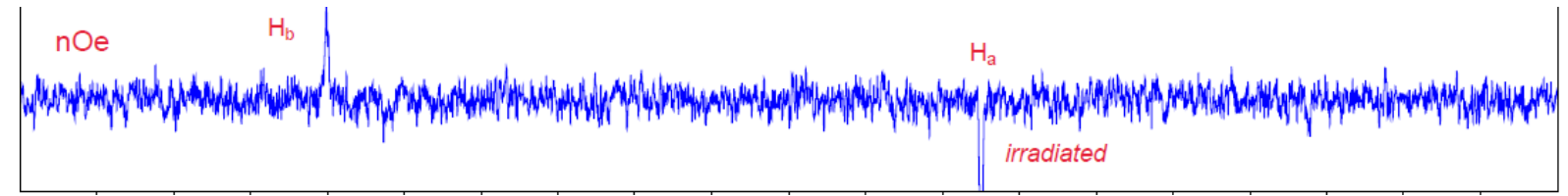

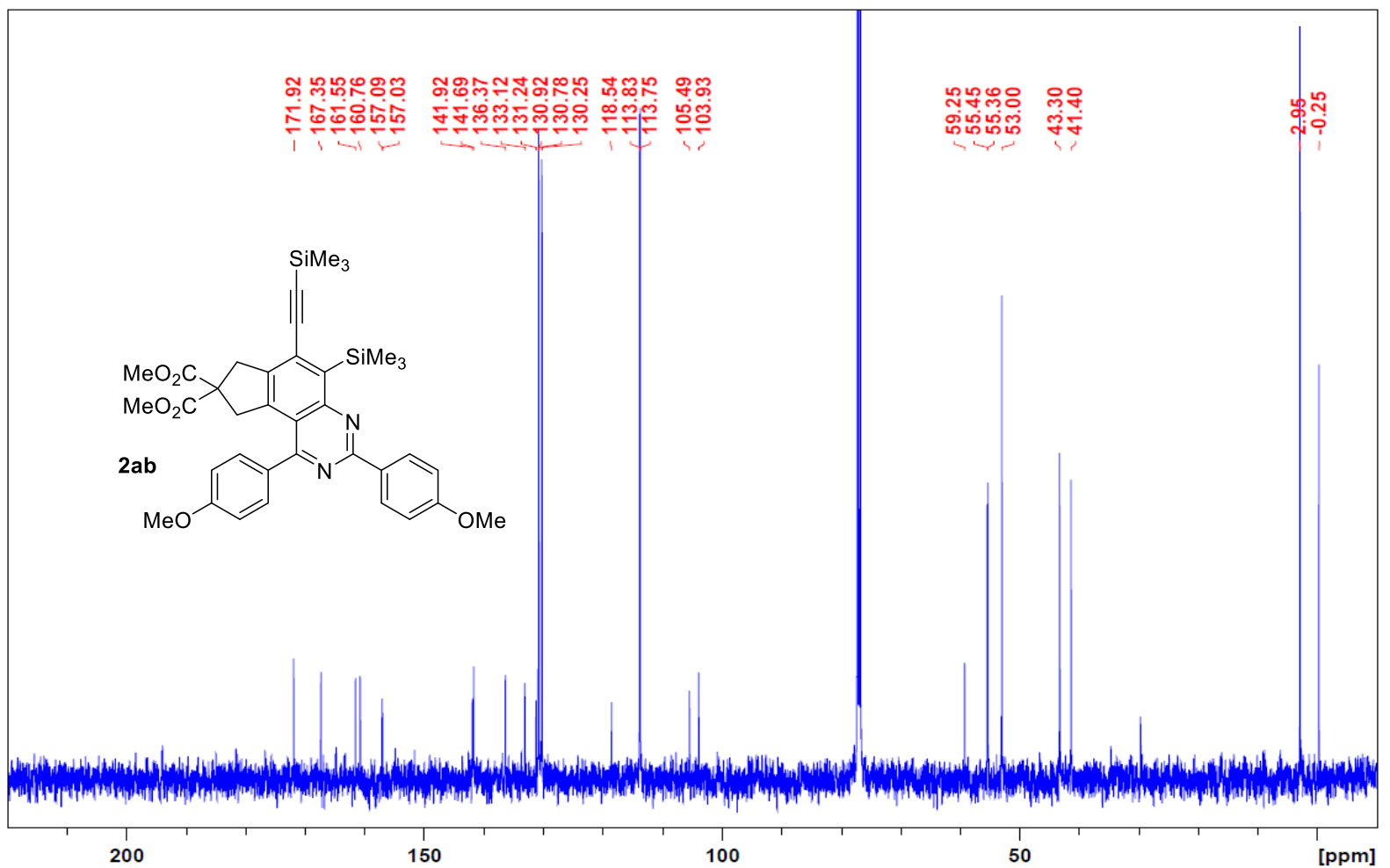



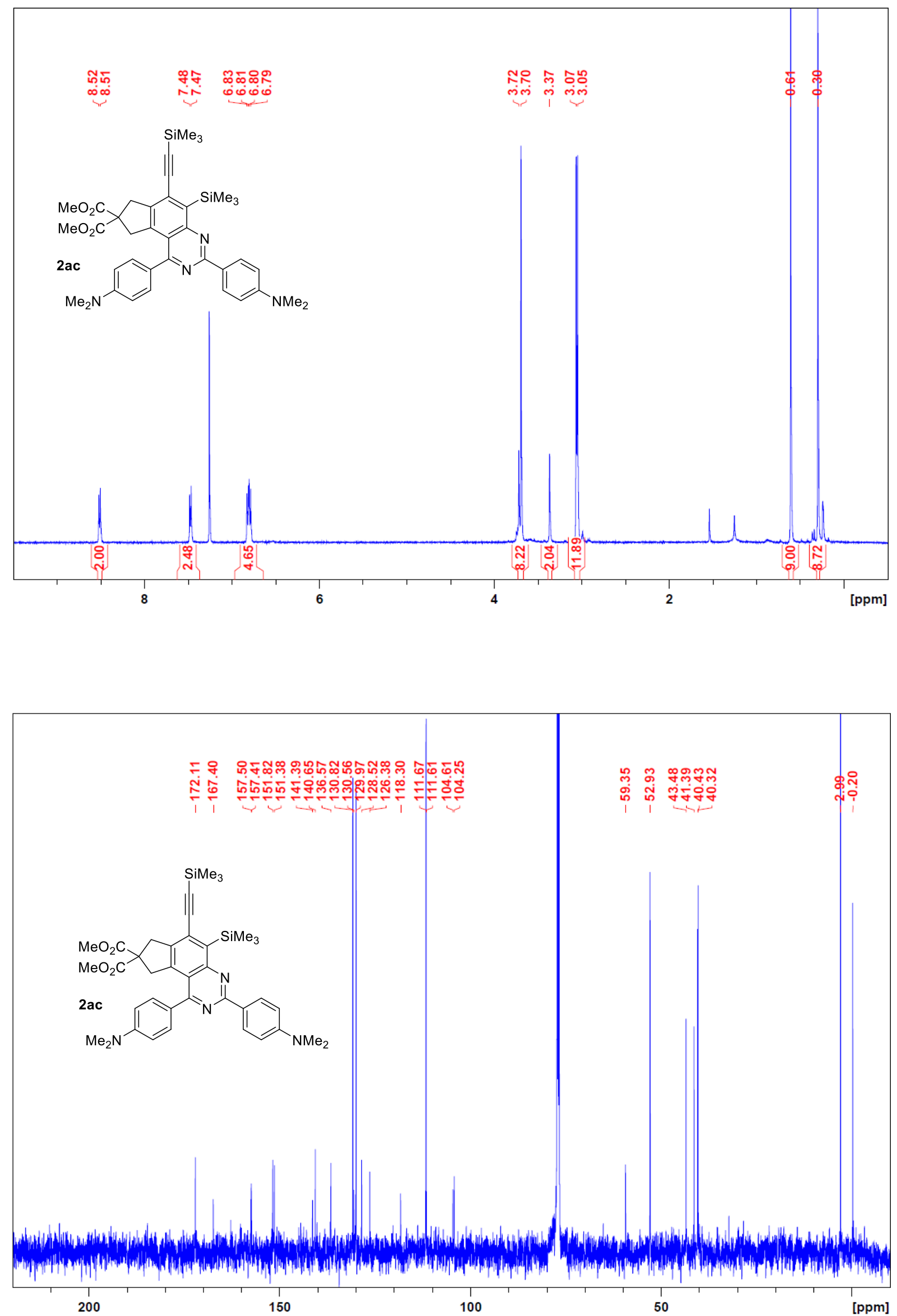

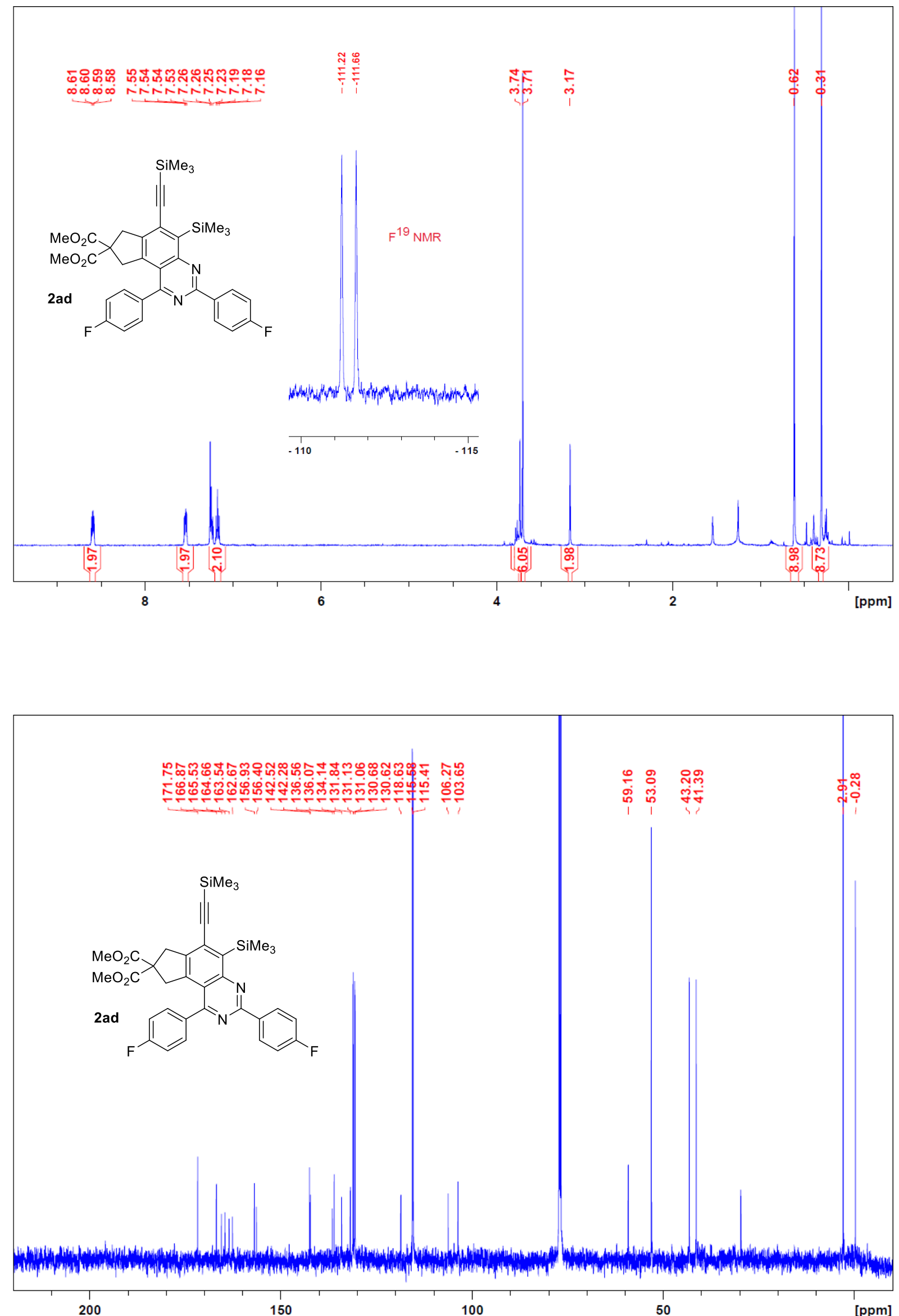

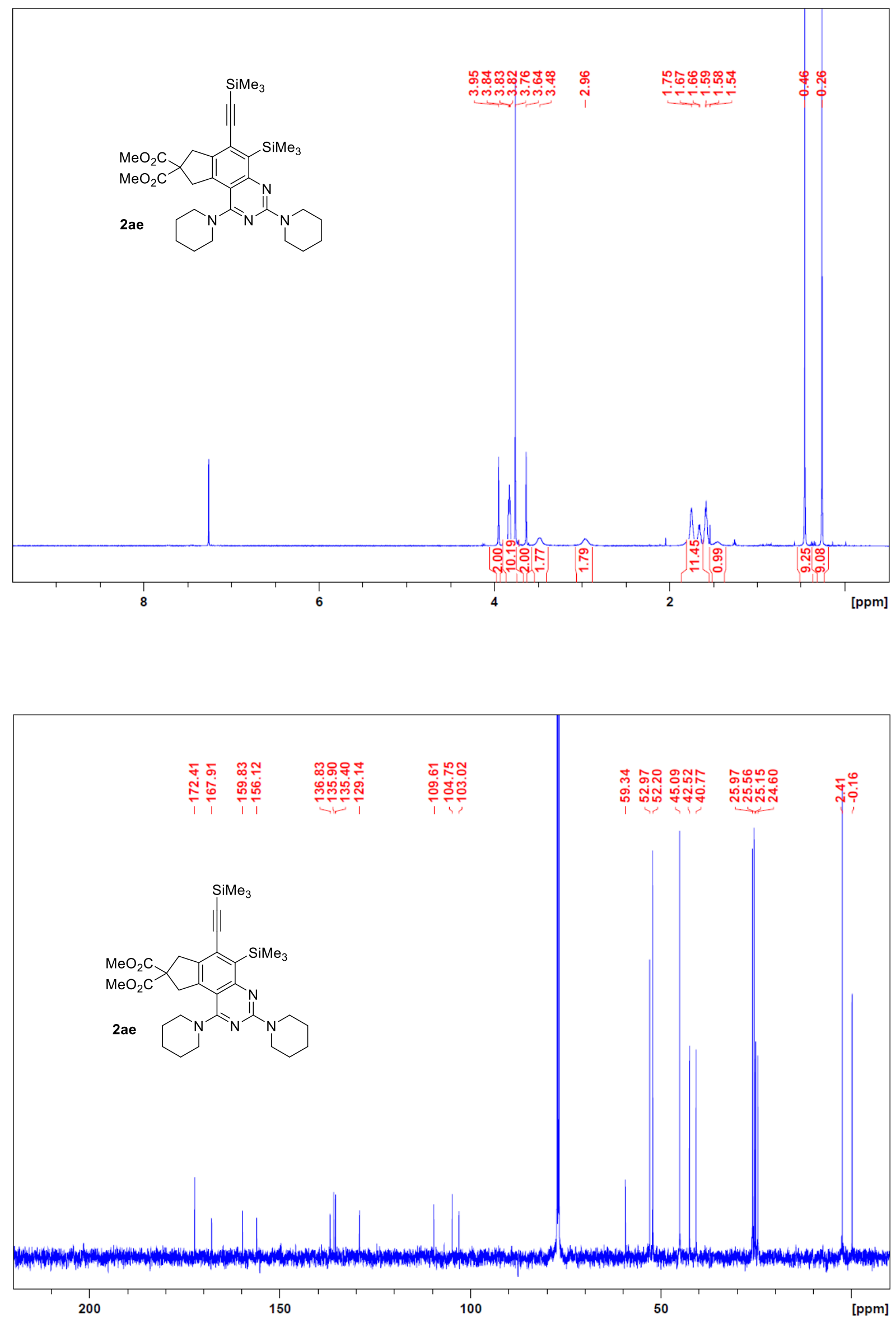

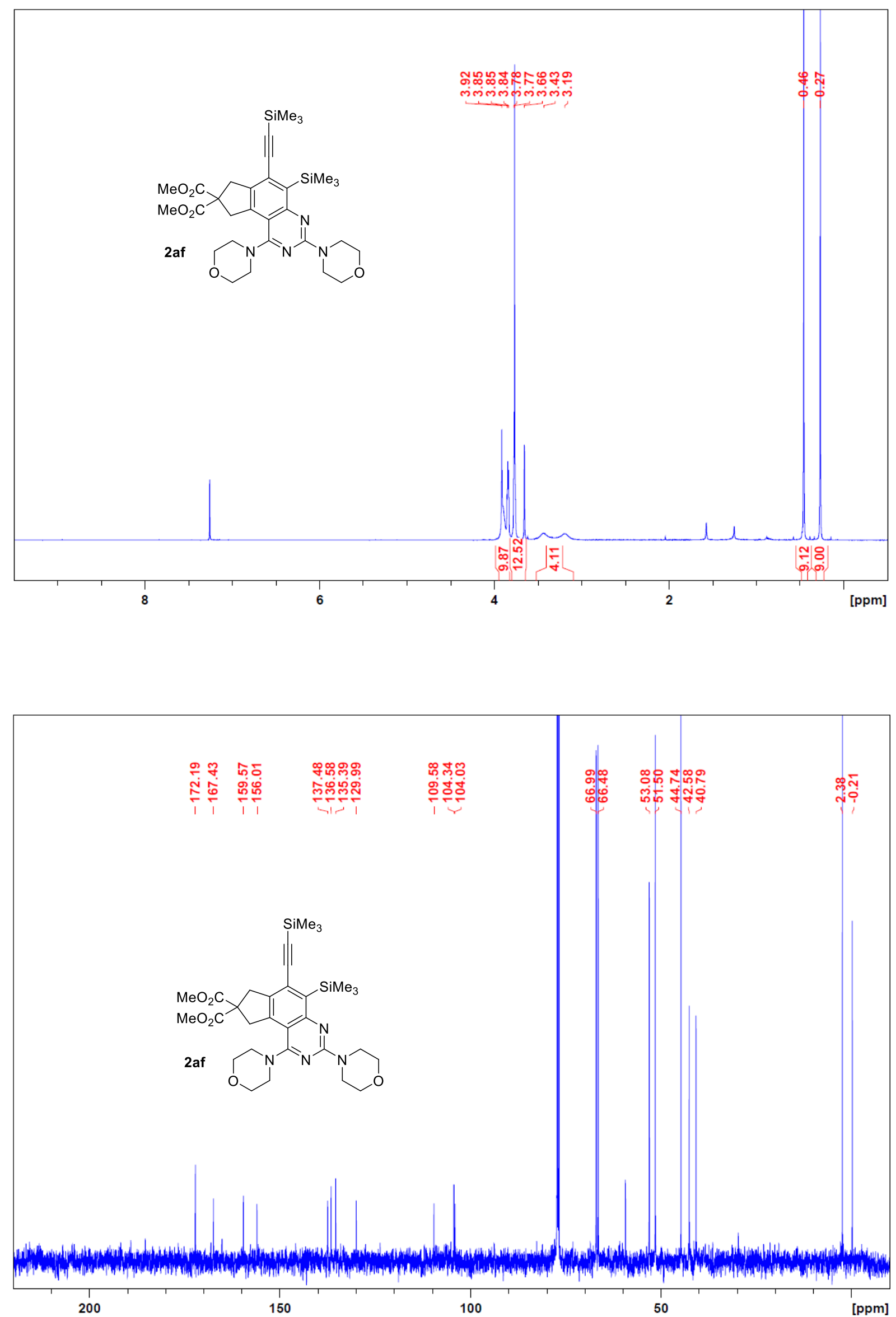

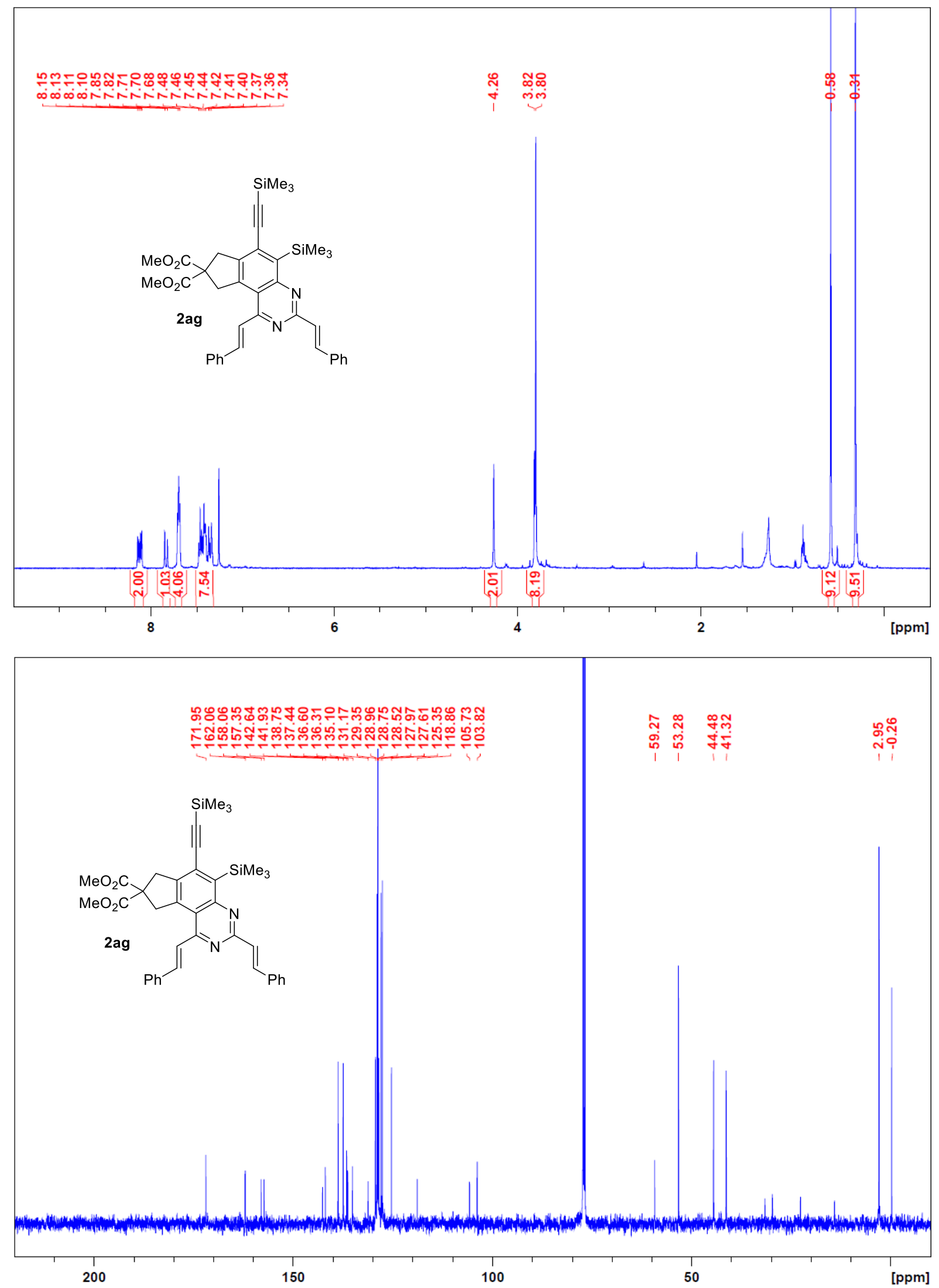

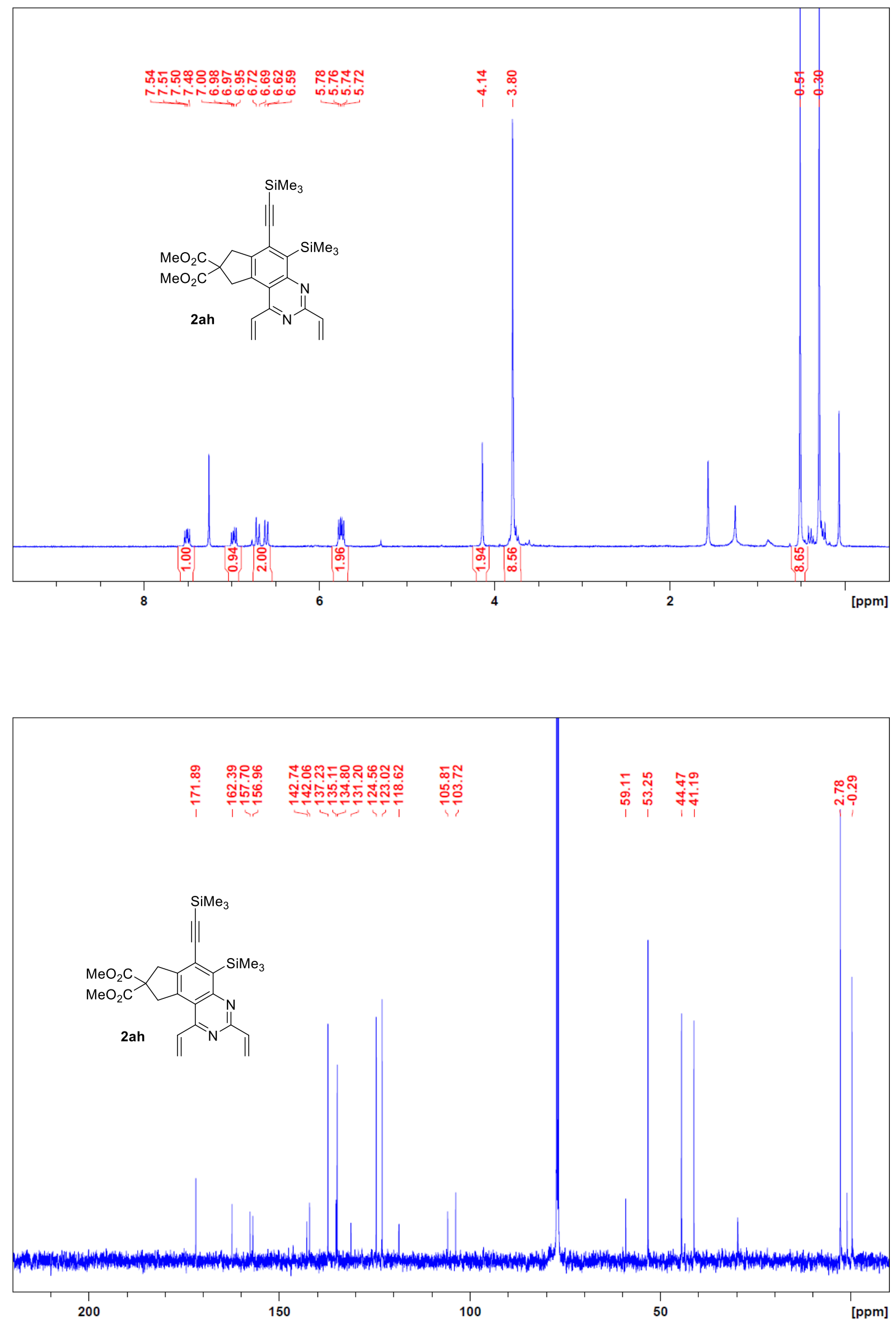

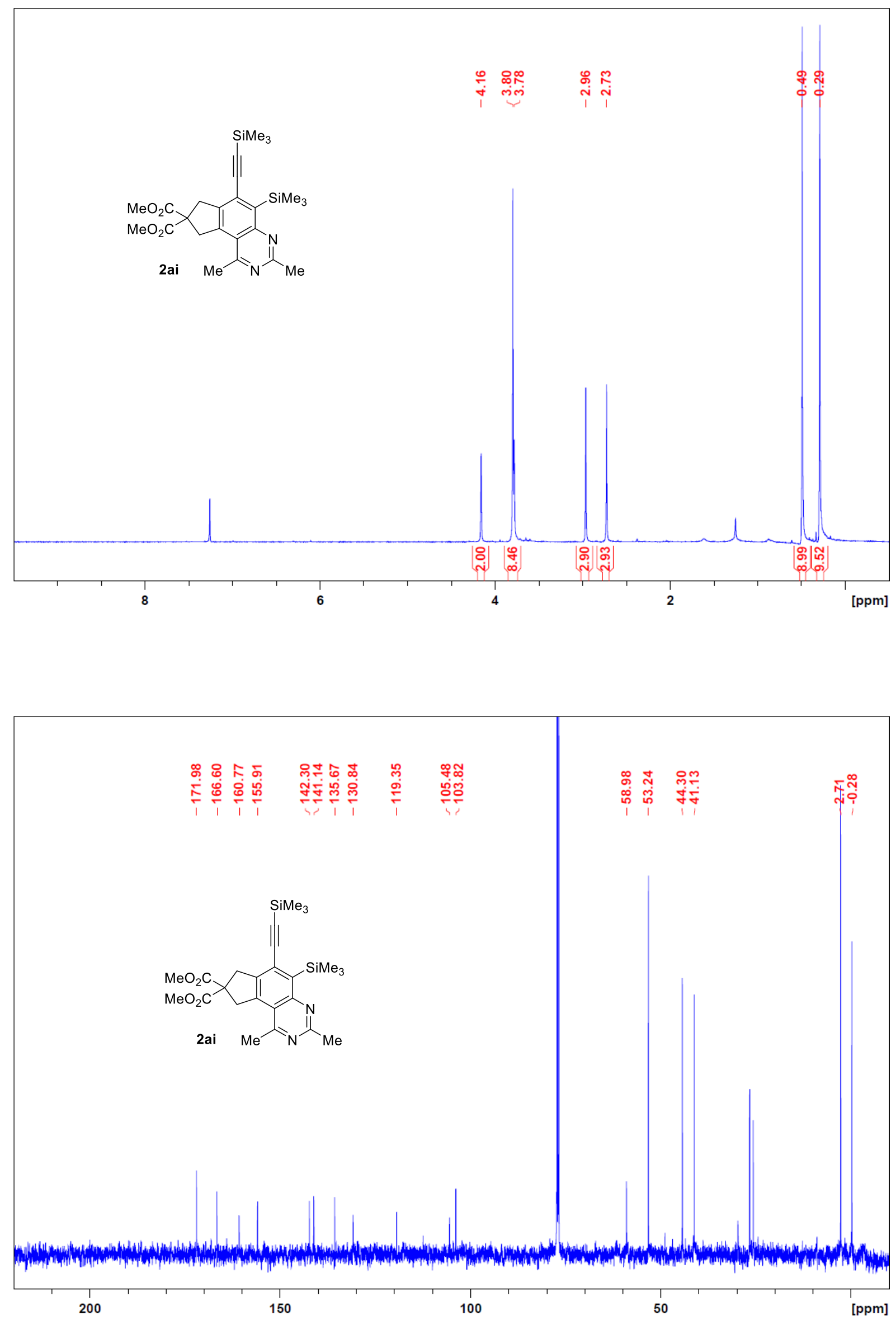

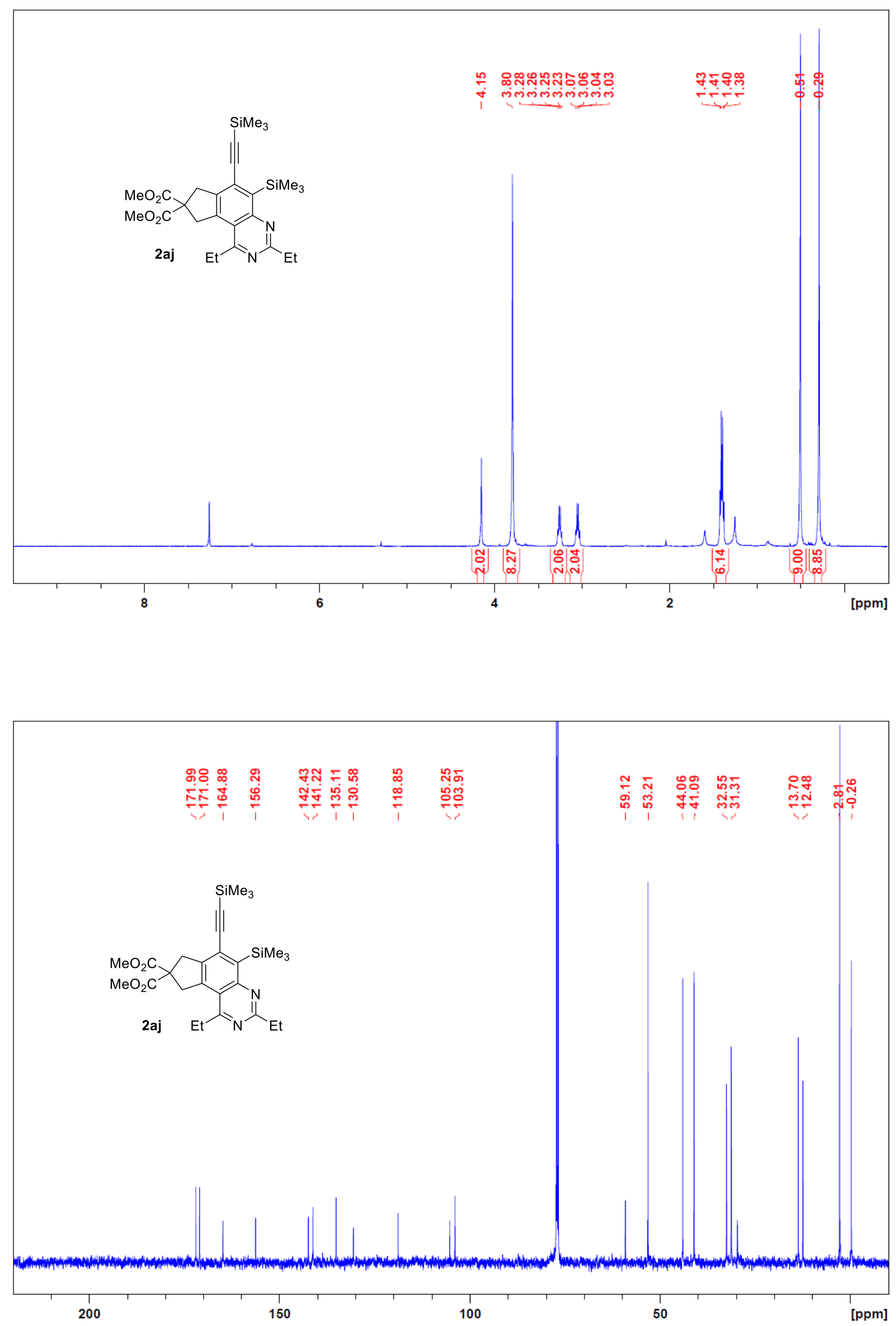

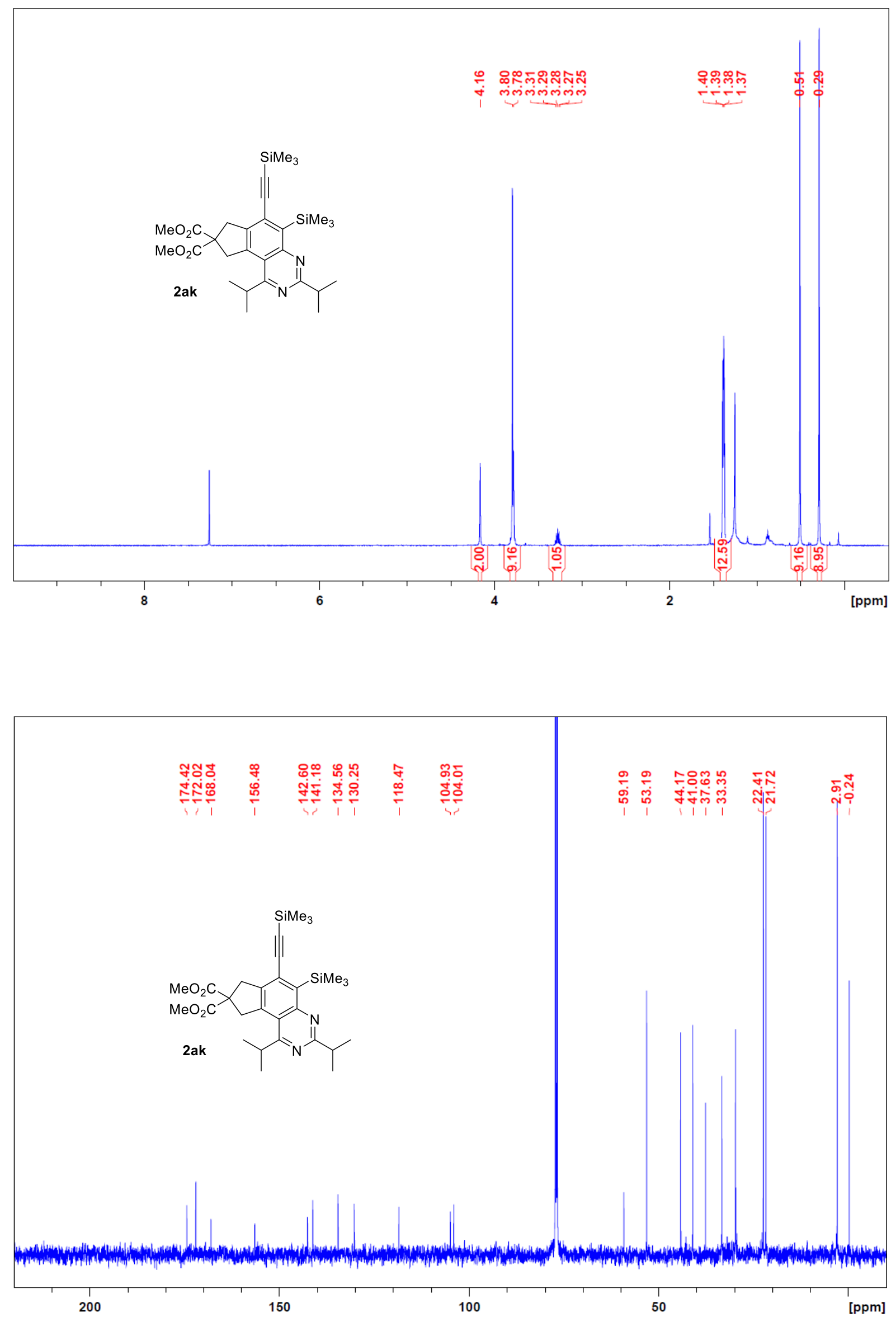

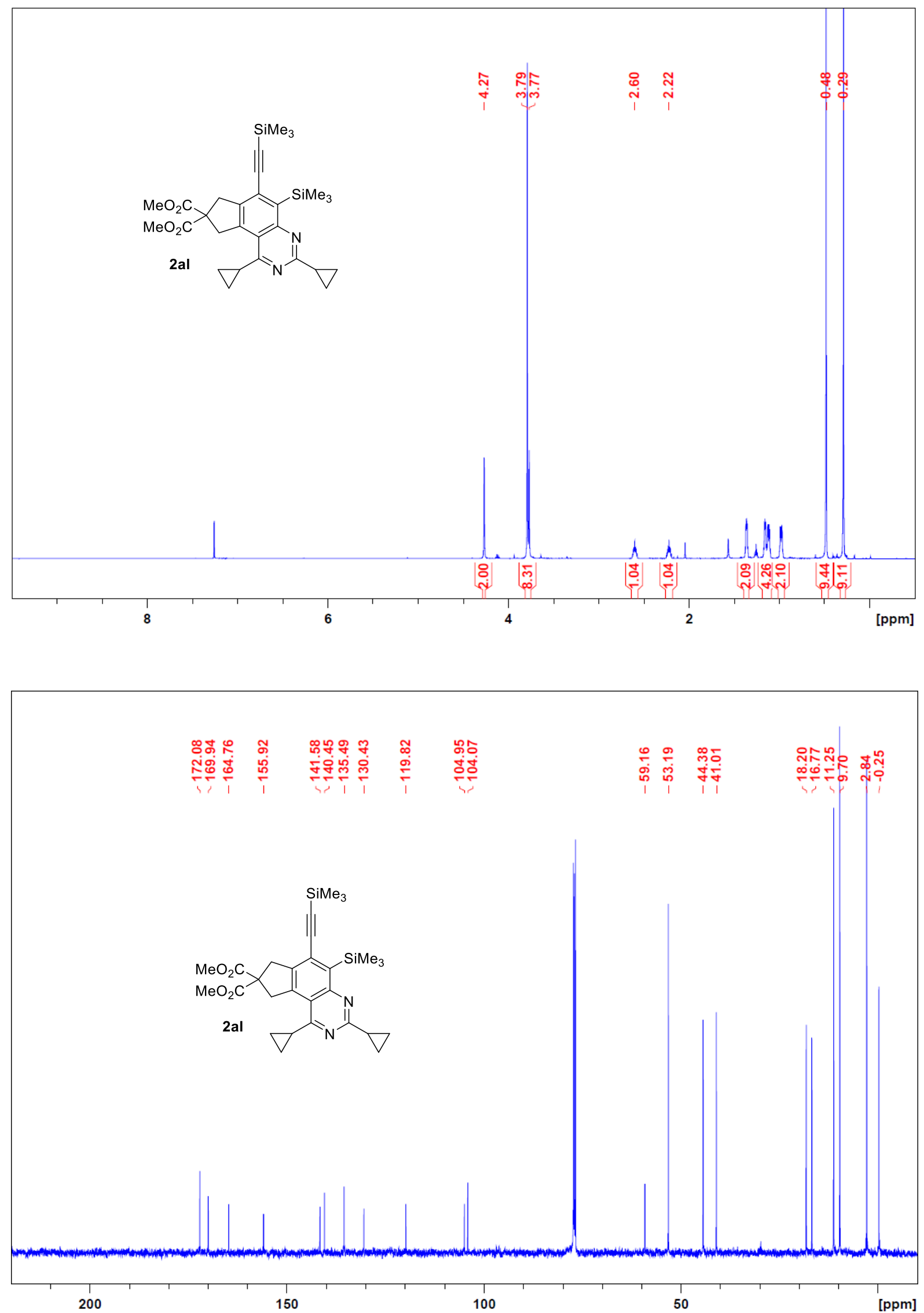

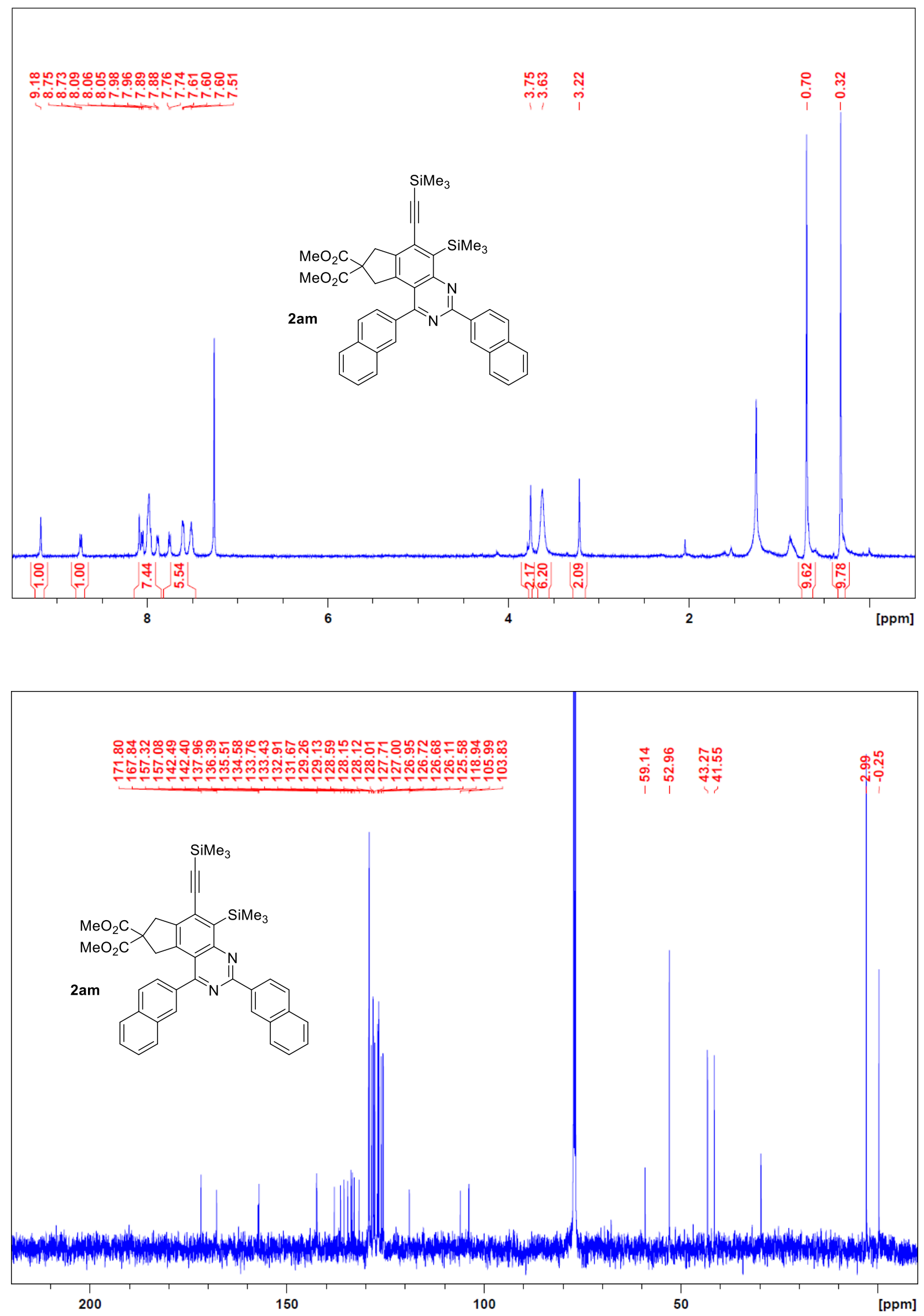

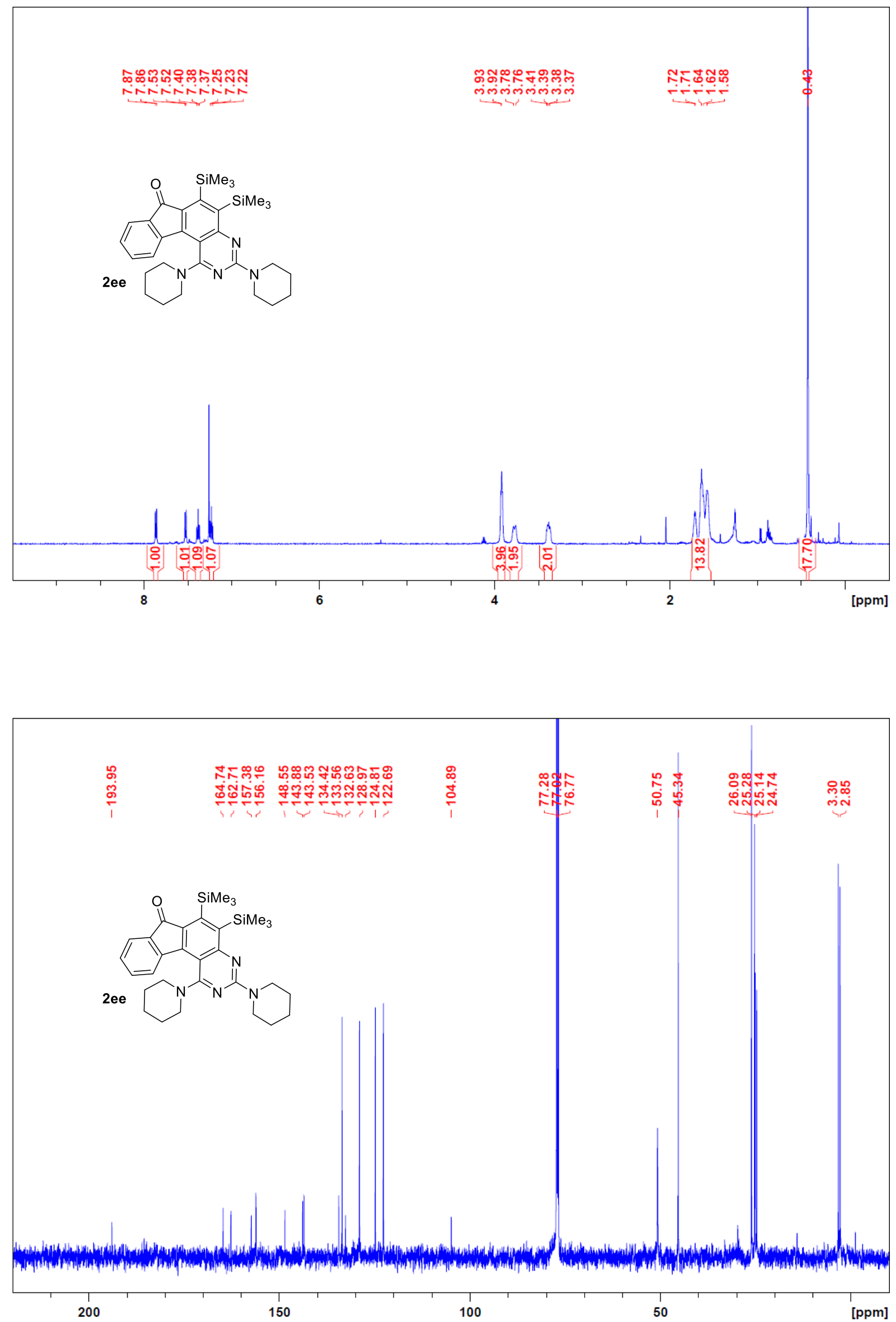

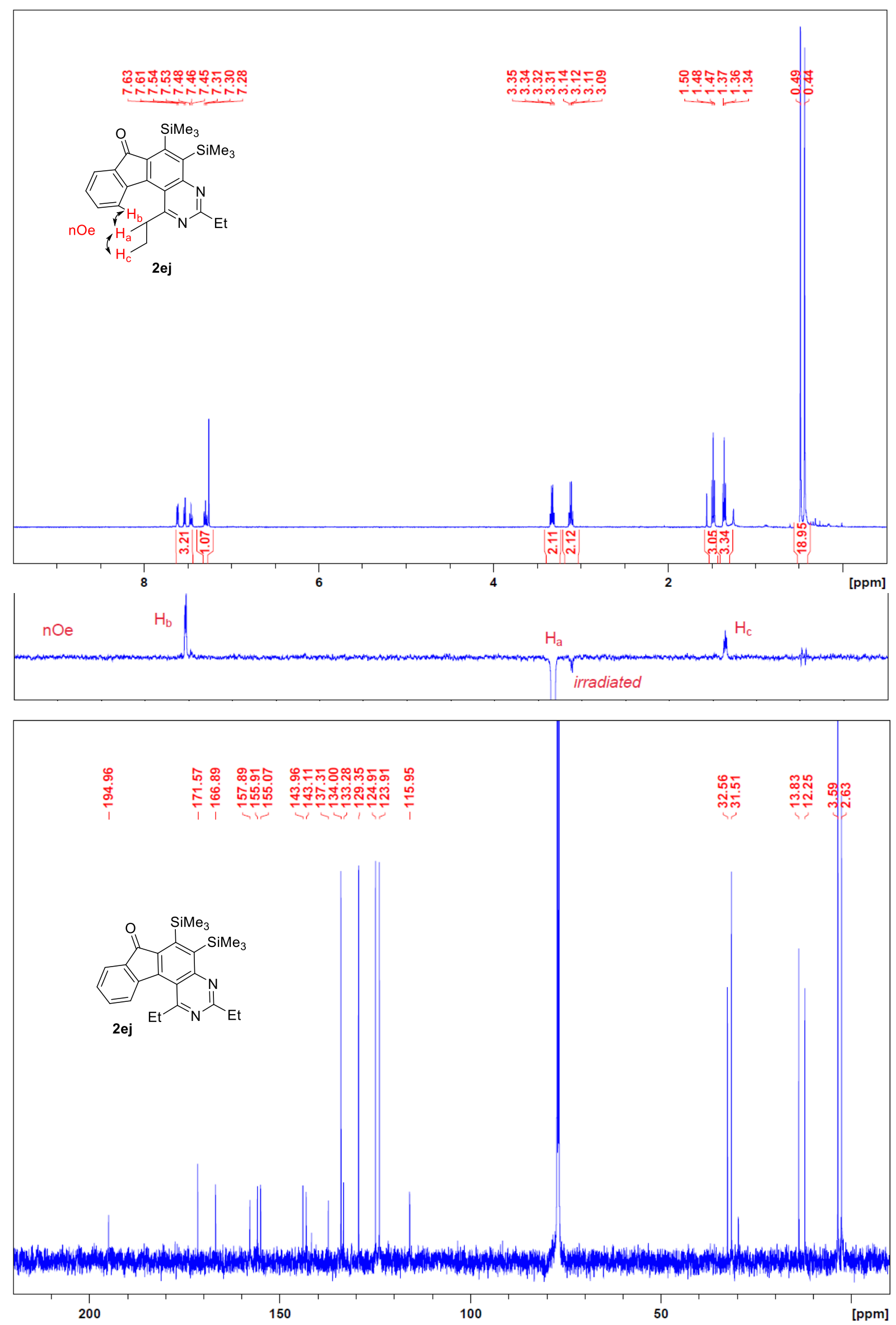

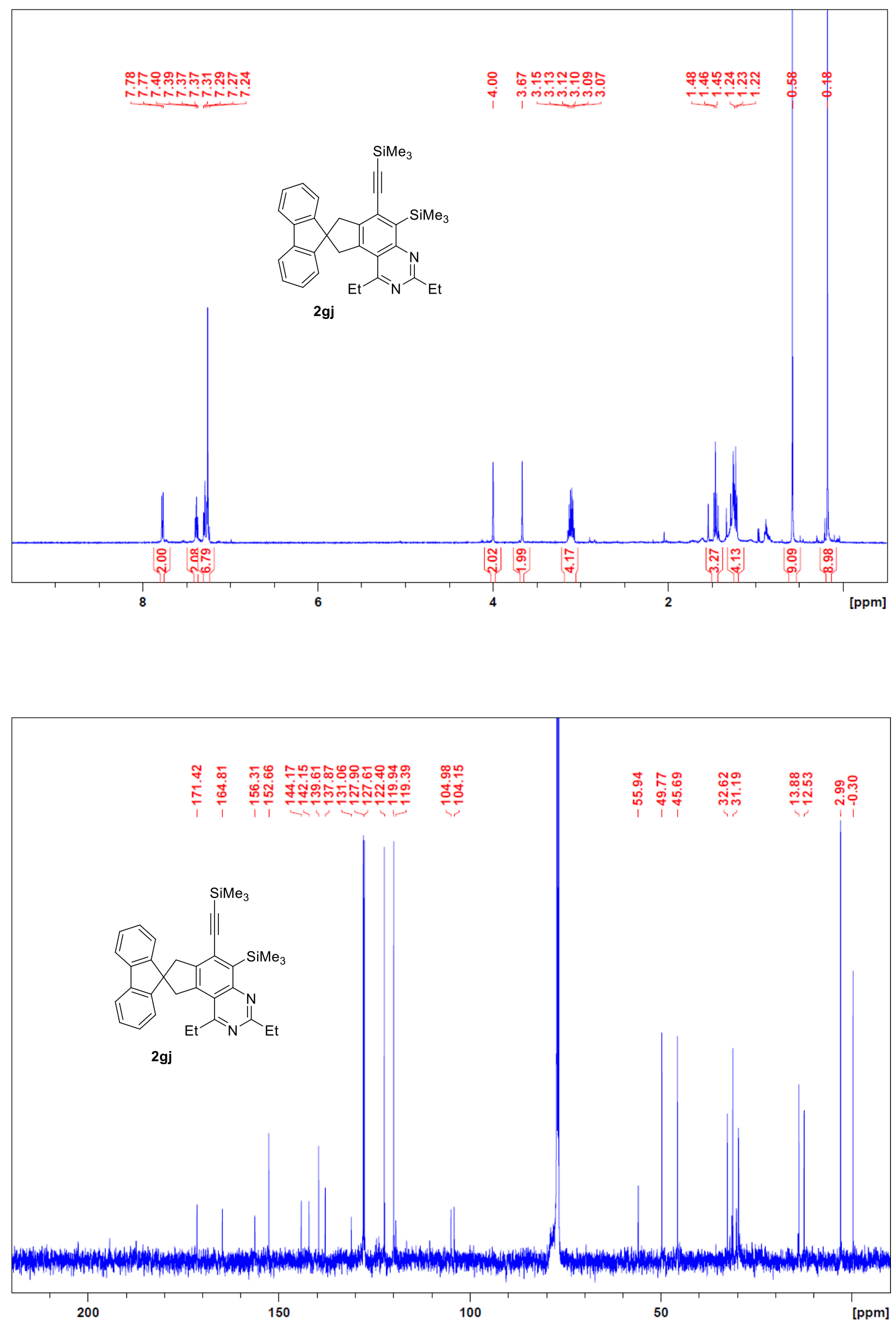

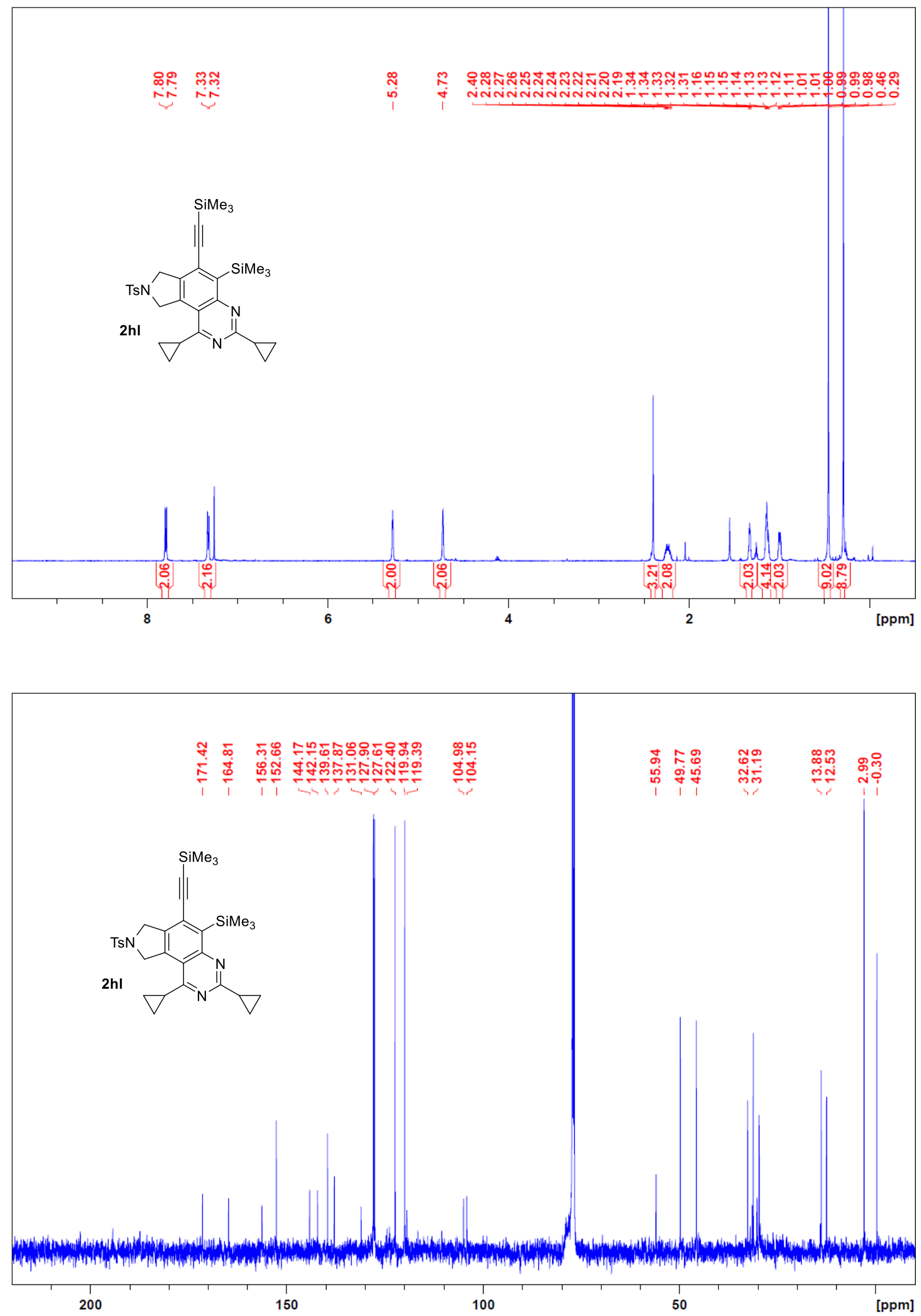

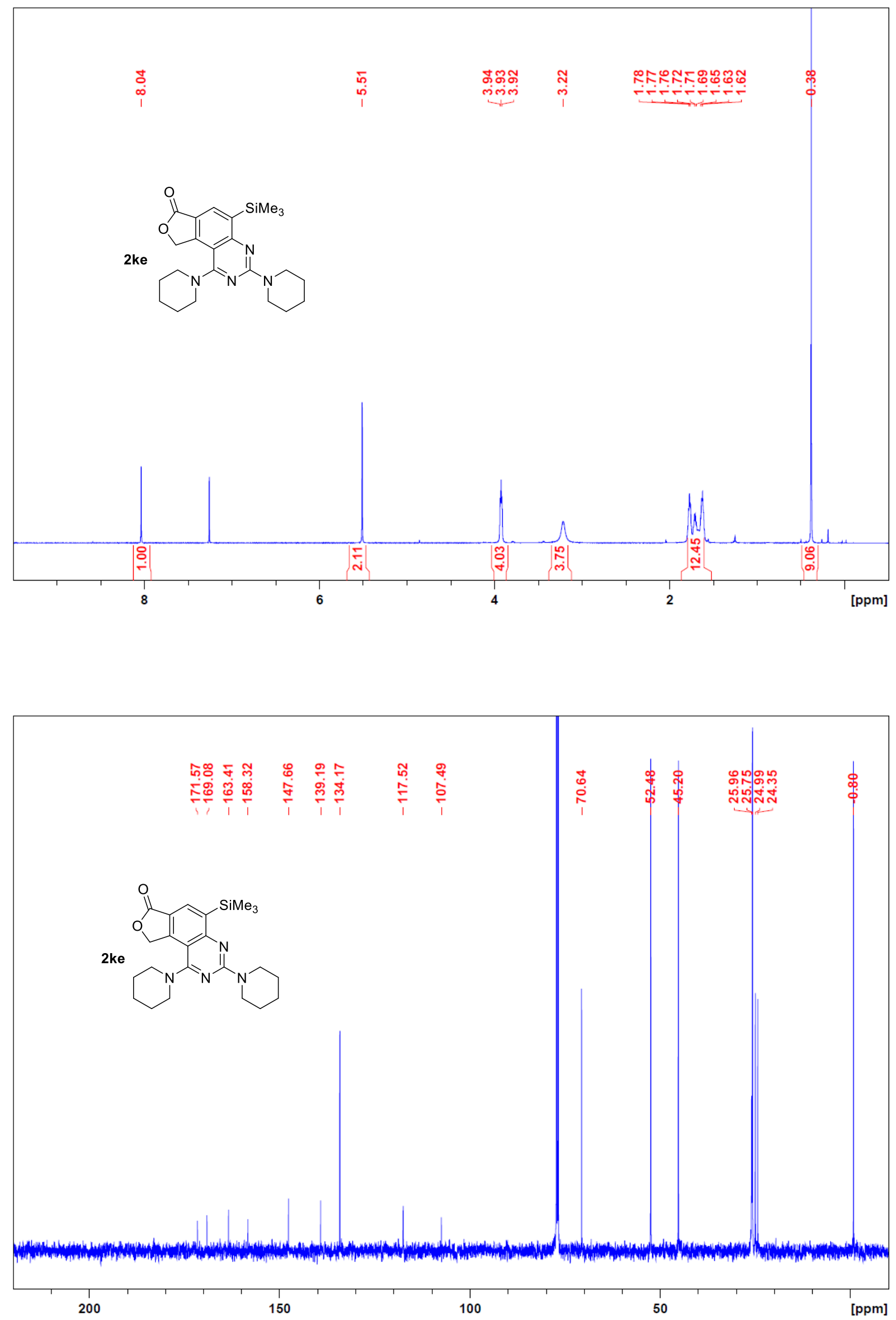

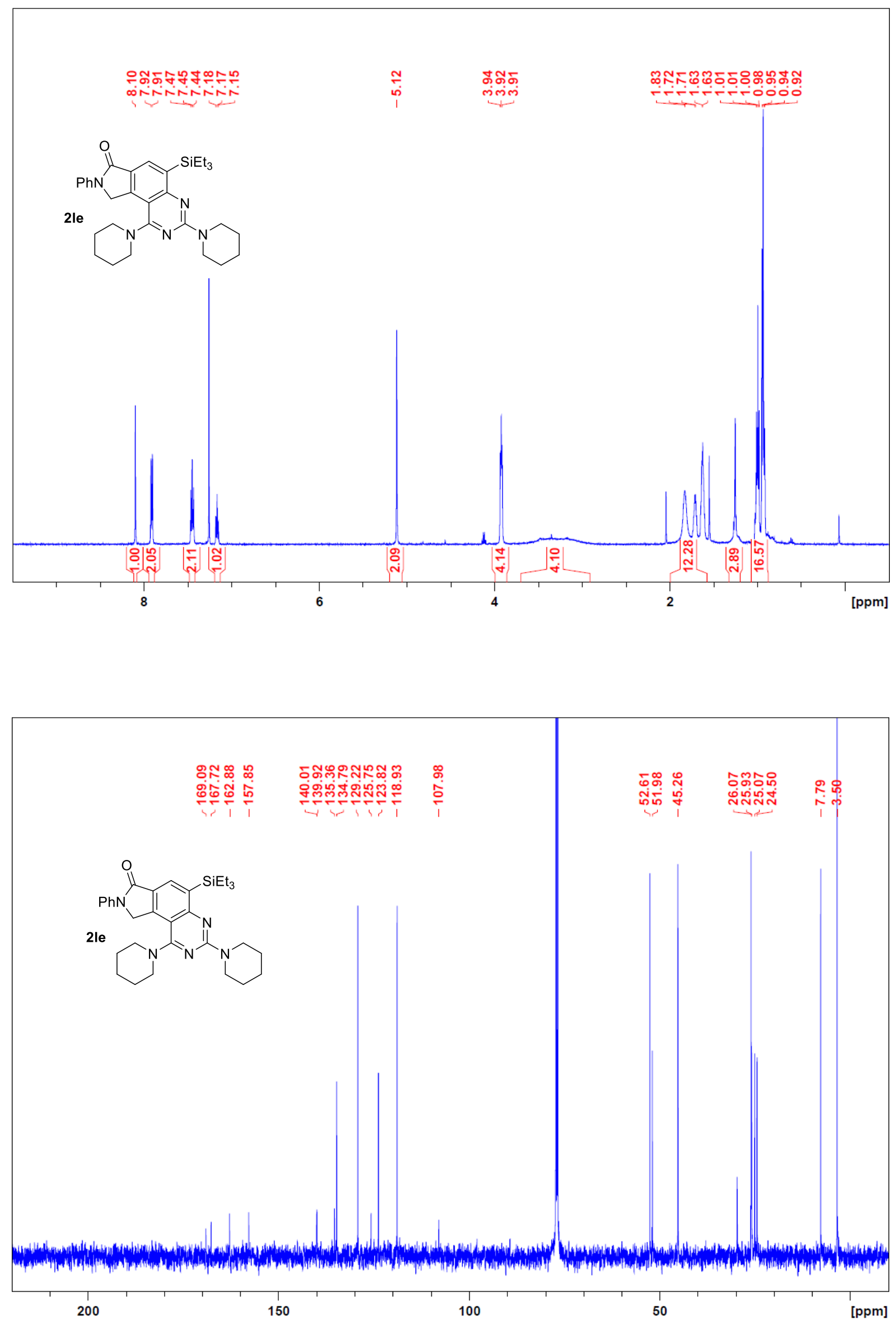

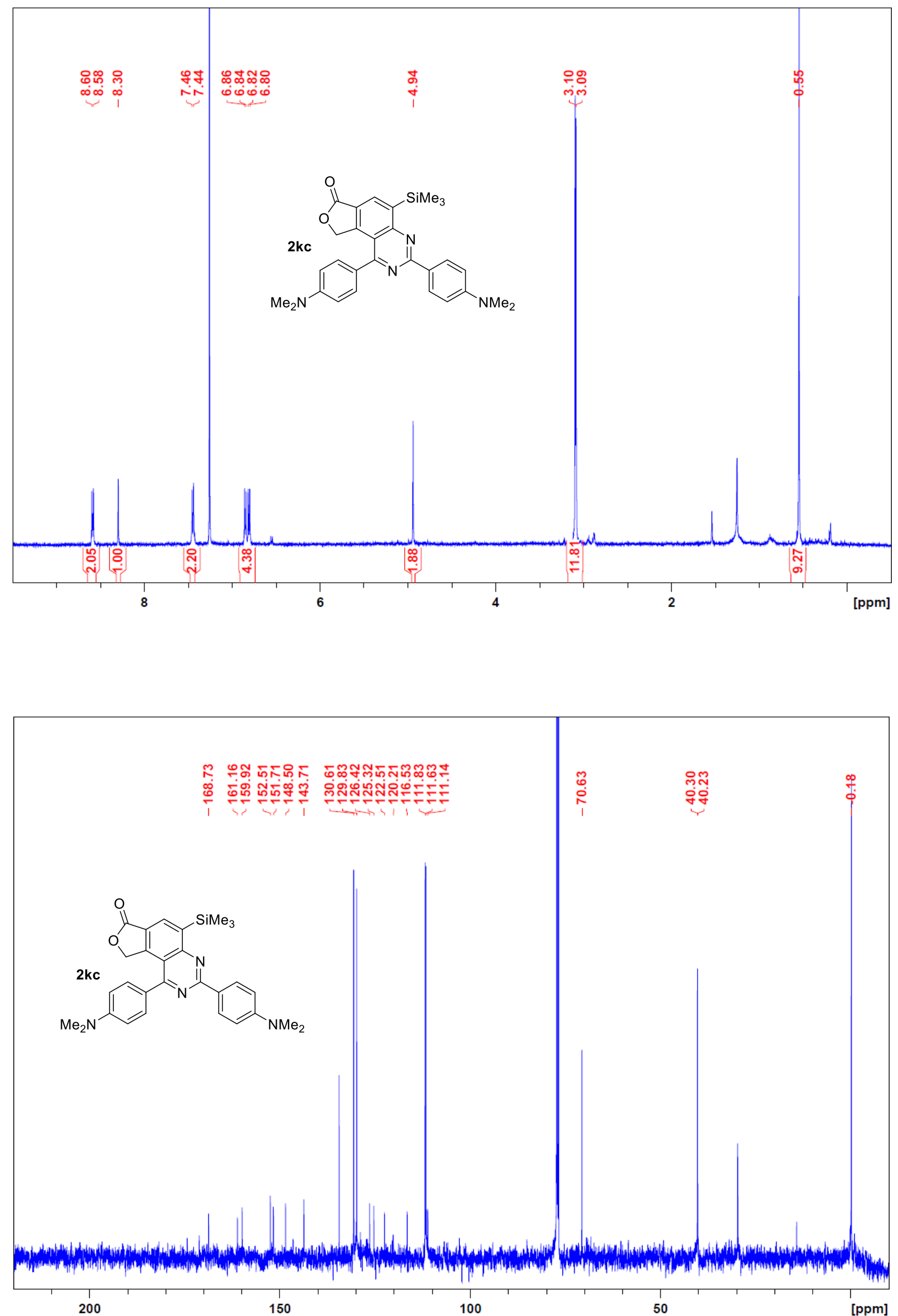

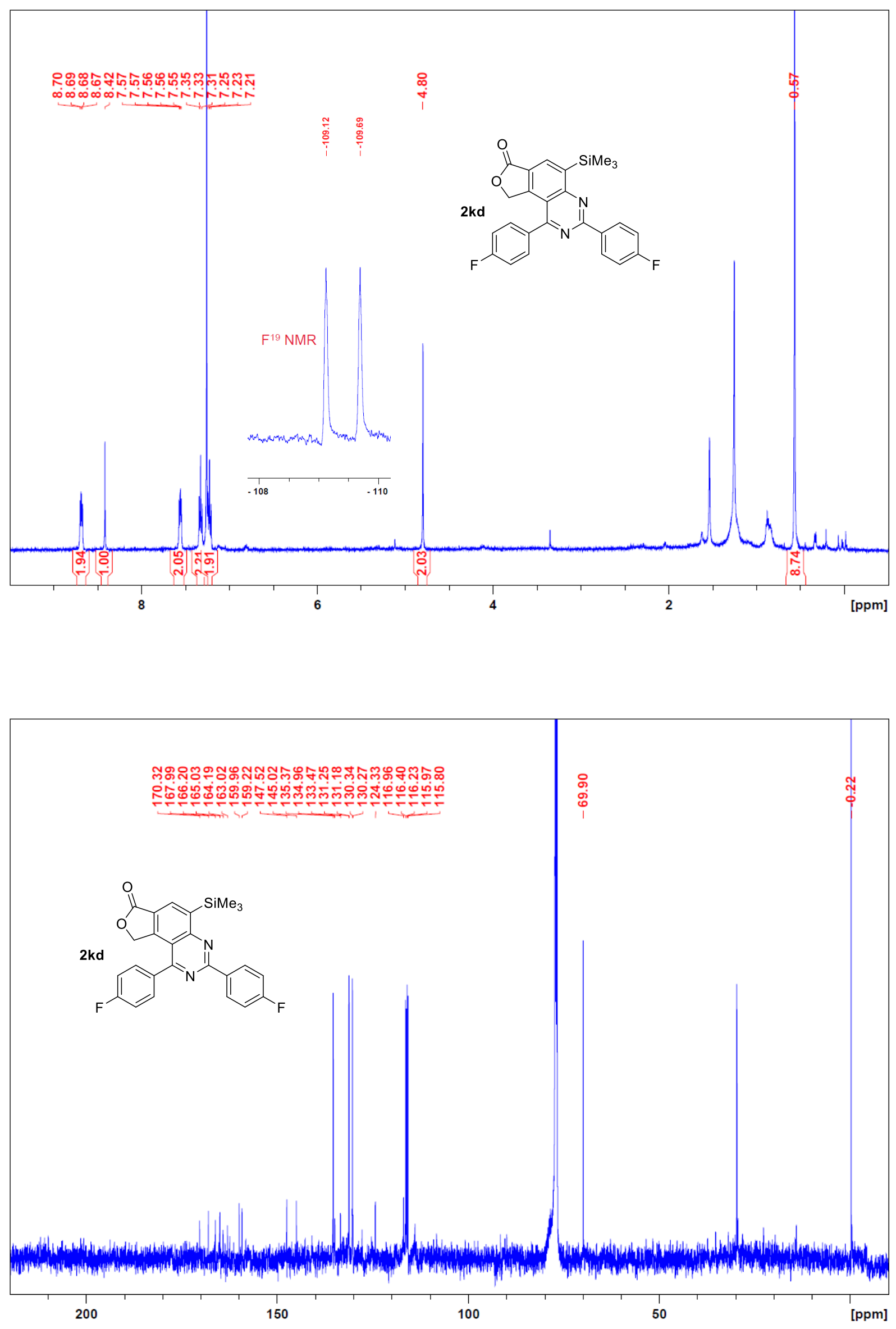

S64 

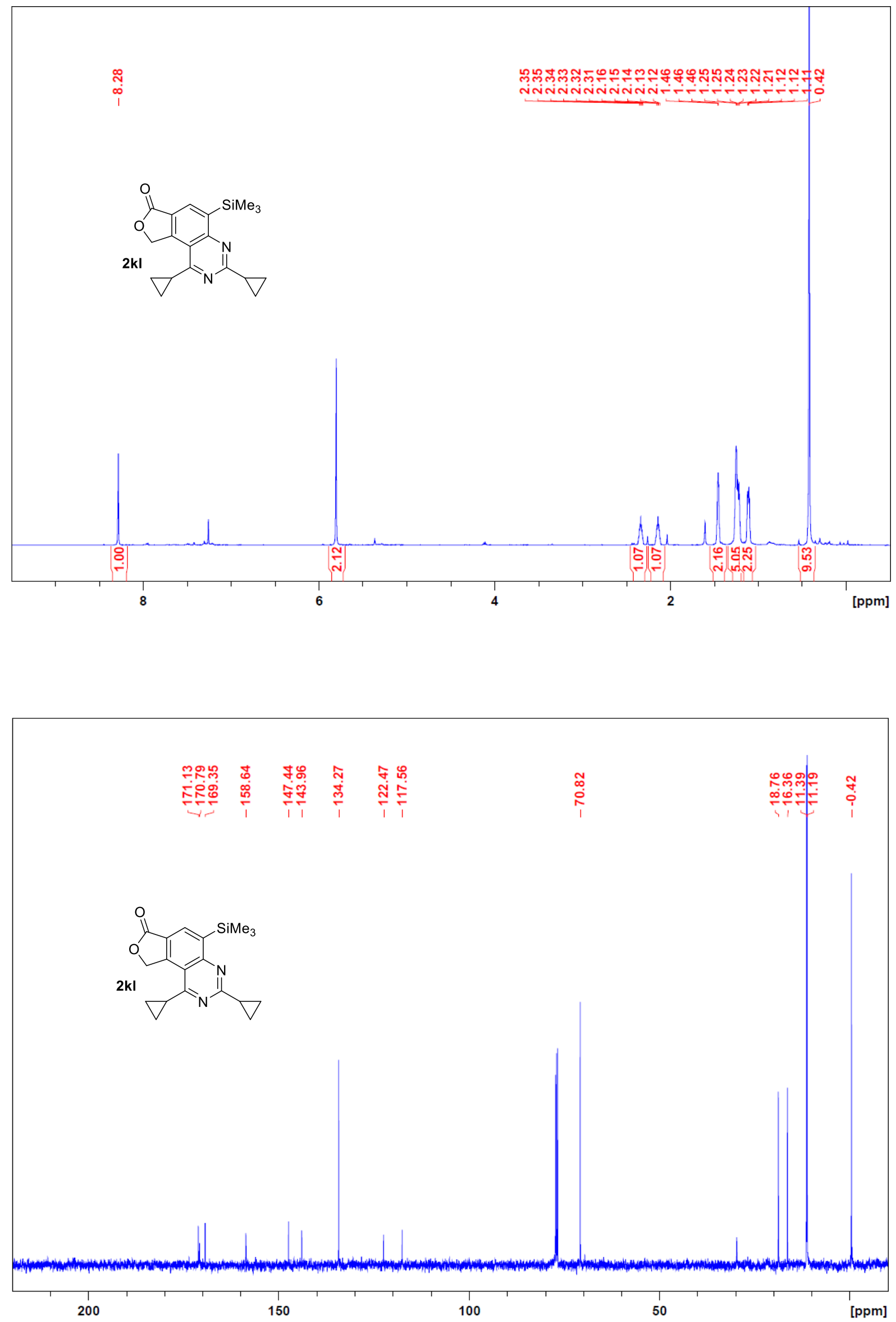


\section{X-Ray Structures of Quinazoline 2ca and 2ka}

CCDC 1903501 (2ca) and CCDC 1903113 (2ka) contain the supplementary crystallographic data for this paper. These data can be obtained free of charge from The Cambridge Crystallographic Data Centre via www.ccdc.cam.ac.uk/data request/cif.
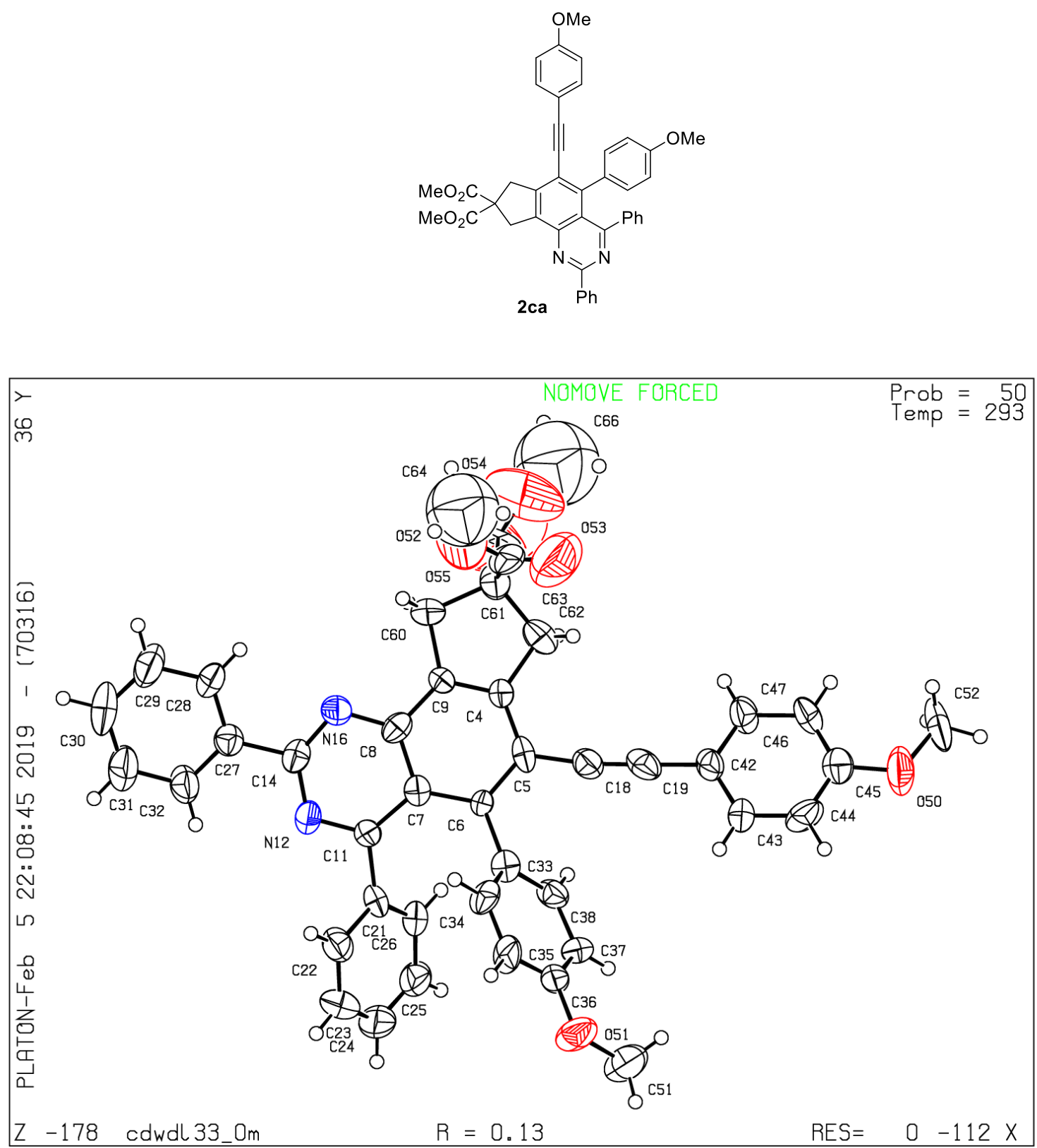

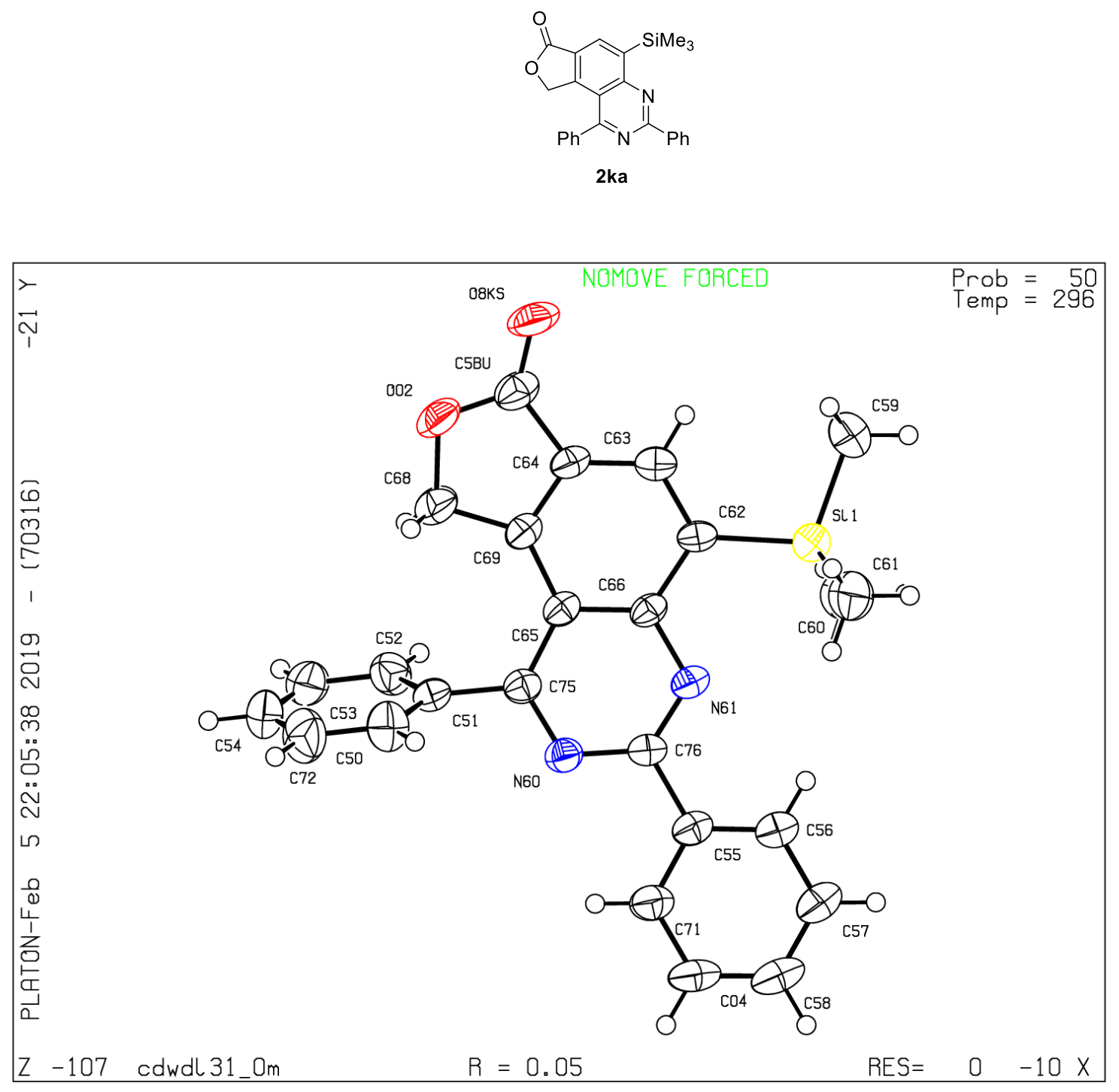


\section{Computational Details}

All calculations were carried out with the Gaussian 09 suite of computational programs. ${ }^{[1]}$ The geometry optimizations and frequency calculations for all intermediates and transition states were done at the B3LYP/6-31G(d) (Lanl2dz for Ag) level of theory. ${ }^{[2]}$ To evaluate the effects of solvent, single point calculations in $\mathrm{PhCN}$ solvent were done at a higher level of M06/6-311++G(d,p) (SDD for Ag) with the SMD model..[3] Unless stated otherwise, all the energy values discussed in the main text are relative free energies in solution $\left(\Delta \mathrm{G}_{\mathrm{sol}}, \mathrm{kcal} / \mathrm{mol}\right)$. Only the intermediate or transition state that has the lowest energy value among all possible conformers is used for discussion.

[1] Gaussian 09, Revision A.01, Frisch, M. J.; Trucks, G. W.; Schlegel, H. B.; Scuseria, G. E.; Robb, M. A.; Cheeseman, J. R.; Scalmani, G.; Barone, V.; Mennucci, B.; Petersson, G. A.; Nakatsuji, H.; Caricato, M.; Li, X.; Hratchian, H. P.; Izmaylov, A. F.; Bloino, J.; Zheng, G.; Sonnenberg, J. L.; Hada, M.; Ehara, M.; Toyota, K.; Fukuda, R.; Hasegawa, J.; Ishida, M.; Nakajima, T.; Honda, Y.; Kitao, O.; Nakai, H.; Vreven, T.; Montgomery, Jr. J. A.; Peralta, J. E.; Ogliaro, F.; Bearpark, M.; Heyd, J. J.; Brothers, E.; Kudin, K. N.; Staroverov, V. N.; Kobayashi, R.; Normand, J.; Raghavachari, K.; Rendell, A.; Burant, J. C.; Iyengar, S. S.; Tomasi, J.; Cossi, M.; Rega, N.; Millam, J. M.; Klene, M.; Knox, J. E.; Cross, J. B.; Bakken, V.; Adamo, C.; Jaramillo, J.; Gomperts, R.; Stratmann, R. E.; Yazyev, O.; Austin, A. J.; Cammi, R.; Pomelli, C.; Ochterski, J. W.; Martin, R. L.; Morokuma, K.; Zakrzewski, V. G.; Voth, G. A.; Salvador, P.; Dannenberg, J. J.; Dapprich, S.; Daniels, A. D.; Farkas, Ö.; Foresman, J. B.; Ortiz, J. V.; Cioslowski, J.; Fox, D. J.; Gaussian, Inc., Wallingford CT, 2009.

[2] (a) Becke, A. D. J. Chem. Phys. 1993, 98, 5648; J. Chem. Phys., 1993, 98, 1372. (b) Lee, C.; Yang, W.; Parr, R. G. Phys. Rev. B, 1988, 37, 785.

[3] Zhao, Y.; Truhlar, D. G. Theor. Chem. Acc. 2008, 120, 215; Acc. Chem. Res. 2008, 41, 157. 


\section{DFT-calculated reaction profiles of substrate $1 \mathrm{~h}$ leading to product $2 \mathrm{ha}$}
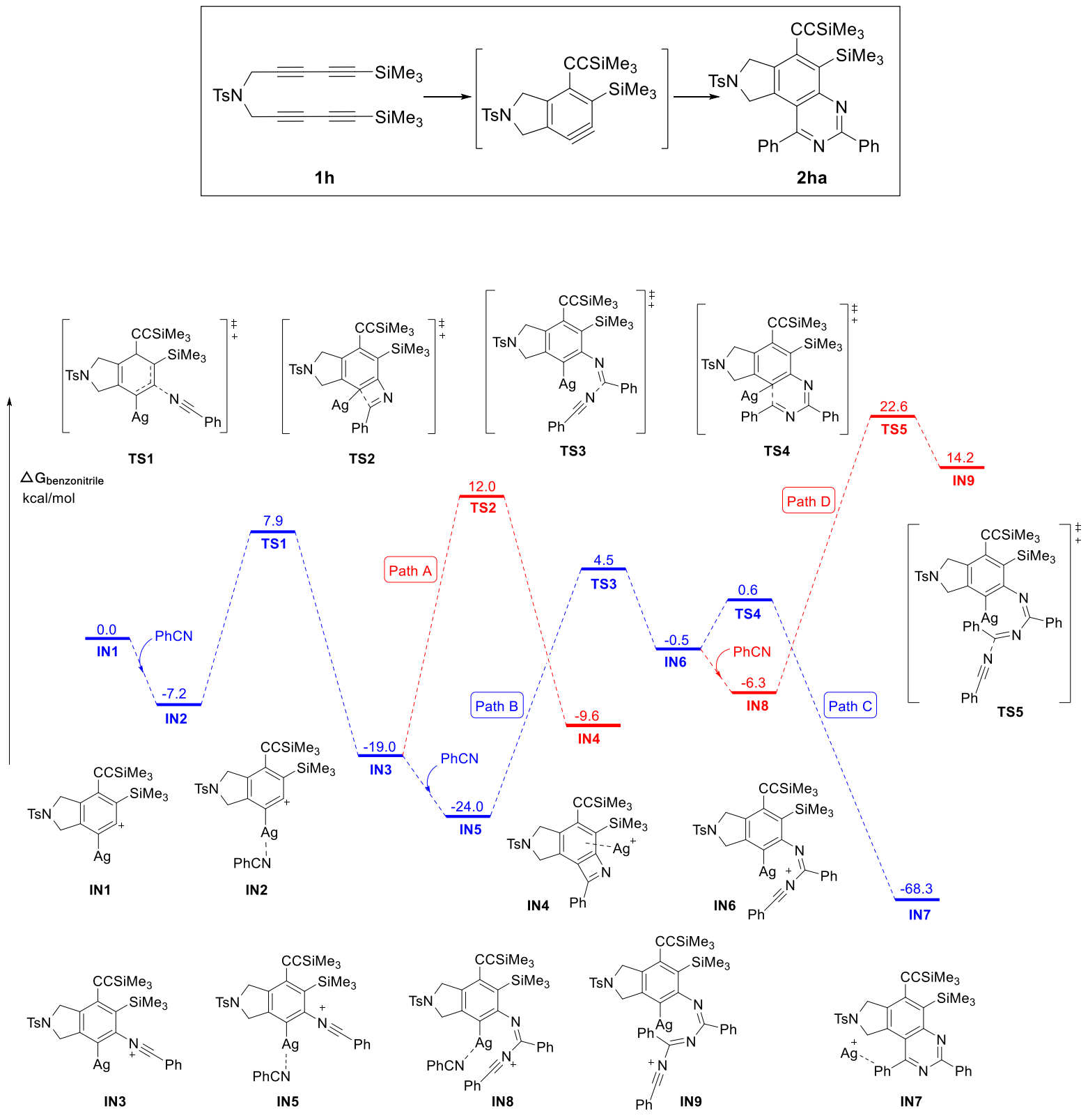


\section{Table of Energy Values (in a.u.)}

Table S1. Energies (in Hartree) calculated at SMD-M06/6-311++G(d,p)/SDD//B3LYP/6$31 \mathrm{G}(\mathrm{d}) / \mathrm{LANL} 2 \mathrm{DZ}$

\begin{tabular}{|c|c|c|c|}
\hline Species & $\mathrm{G}_{298}{ }^{\mathrm{a}}$ & $\mathrm{E}^{\mathrm{b}}$ & Gsol $^{\mathrm{c}}$ \\
\hline IN1 & -2221.303242 & -2221.6892597 & -2222.49967543 \\
\hline IN2 & -2545.768723 & -2546.2382366 & -2546.86137157 \\
\hline TS1 & -2545.717761 & -2546.1909057 & -2546.84089336 \\
\hline IN3 & -2545.762602 & -2546.2428241 & -2546.89097255 \\
\hline TS2 & -2545.714839 & -2546.2000087 & -2546.84644963 \\
\hline IN4 & -2545.755738 & -2546.2408277 & -2546.88083133 \\
\hline IN5 & -2870.213261 & -2870.7805772 & -2871.2526512 \\
\hline TS3 & -2870.151831 & -2870.7223625 & -2871.21046332 \\
\hline IN6 & -2870.162116 & -2870.7362036 & -2871.22194566 \\
\hline TS4 & -2870.151587 & -2870.72675 & -2871.22136809 \\
\hline IN7 & -2870.269461 & -2870.8526612 & -2871.33920379 \\
\hline IN8 & -3194.606568 & -3195.2686842 & -3195.58607526 \\
\hline TS5 & -3194.548634 & -3195.2142097 & -3195.54344772 \\
\hline IN9 & -3194.555495 & -3195.2254724 & -3195.56124176 \\
\hline
\end{tabular}

${ }^{a}$ Sum of electronic and thermal free energies

${ }^{\mathrm{b}}$ Electronic energies

${ }^{\mathrm{c}}$ Single point energies in solution 


\section{Cartesian Coordinates}

\begin{tabular}{|c|c|c|c|c|c|c|c|}
\hline IN1 & & & & $\mathrm{H}$ & -3.55038000 & -0.48392200 & -0.66534200 \\
\hline $\mathrm{C}$ & -3.25978100 & 0.67779300 & -0.33584100 & $\mathrm{C}$ & -0.98481400 & -0.47068300 & 1.05131600 \\
\hline $\mathrm{H}$ & -3.82799900 & 1.59204000 & -0.53427800 & $\mathrm{H}$ & -0.38390000 & 0.00309700 & 1.83543200 \\
\hline $\mathrm{H}$ & -3.89874800 & -0.18776000 & -0.58629300 & $\mathrm{H}$ & -0.95712100 & -1.55839400 & 1.21000400 \\
\hline $\mathrm{C}$ & -1.41374700 & 0.11066200 & 1.16604400 & $\mathrm{C}$ & -1.59900400 & 0.32345800 & -1.10535400 \\
\hline $\mathrm{H}$ & -0.83639700 & 0.67554400 & 1.90291500 & $\mathrm{C}$ & -0.51722700 & -0.11552600 & -0.33462300 \\
\hline $\mathrm{H}$ & -1.40232900 & -0.95032600 & 1.46631500 & $\mathrm{C}$ & -1.44264100 & 0.69820800 & -2.48284200 \\
\hline $\mathrm{C}$ & -1.95866600 & 0.60628500 & -1.09997600 & $\mathrm{C}$ & 0.79492000 & -0.21514200 & -0.84938800 \\
\hline $\mathrm{C}$ & -0.90127700 & 0.27256600 & -0.24244600 & $\mathrm{C}$ & -0.16747300 & 0.51253600 & -2.67898700 \\
\hline $\mathrm{C}$ & -1.74361500 & 0.82446300 & -2.49774100 & $\mathrm{C}$ & 1.86271100 & -0.66439700 & -0.03004500 \\
\hline $\mathrm{C}$ & 0.42969000 & 0.12454100 & -0.69116700 & $\mathrm{C}$ & 1.03760900 & 0.15619900 & -2.24241400 \\
\hline $\mathrm{C}$ & -0.45717600 & 0.62370500 & -2.63114700 & $\mathrm{C}$ & 2.74802300 & -1.05646700 & 0.71728400 \\
\hline $\mathrm{C}$ & 1.46511200 & -0.21648400 & 0.21327700 & $\mathrm{Si}$ & 2.71355200 & 0.12907300 & -3.20492400 \\
\hline $\mathrm{C}$ & 0.72664500 & 0.33352700 & -2.11240500 & $\mathrm{C}$ & 3.87825900 & 1.32228800 & -2.33360600 \\
\hline $\mathrm{C}$ & 2.31577200 & -0.50990600 & 1.04253500 & $\mathrm{H}$ & 4.85863900 & 1.31053100 & -2.82592400 \\
\hline $\mathrm{Si}$ & 2.45082600 & 0.22331000 & -2.99916200 & $\mathrm{H}$ & 3.50111400 & 2.35045600 & -2.36915000 \\
\hline $\mathrm{C}$ & 3.56139300 & 1.51112800 & -2.19957700 & $\mathrm{H}$ & 4.02571300 & 1.04893800 & -1.28410600 \\
\hline $\mathrm{H}$ & 4.55885700 & 1.46819200 & -2.65478300 & $\mathrm{C}$ & 2.29533900 & 0.71583900 & -4.94824200 \\
\hline $\mathrm{H}$ & 3.17367700 & 2.52524700 & -2.34675800 & $\mathrm{H}$ & 1.57195800 & 0.05498200 & -5.44104400 \\
\hline $\mathrm{H}$ & 3.67570100 & 1.34072700 & -1.12472500 & $\mathrm{H}$ & 1.89127100 & 1.73540400 & -4.95315200 \\
\hline $\mathrm{C}$ & 2.09254000 & 0.62869300 & -4.80514900 & $\mathrm{H}$ & 3.19944000 & 0.72404000 & -5.56876400 \\
\hline $\mathrm{H}$ & 1.39840300 & -0.08928700 & -5.25856700 & $\mathrm{C}$ & 3.33176600 & -1.64710000 & -3.20227700 \\
\hline $\mathrm{H}$ & 1.67968300 & 1.63757000 & -4.92593700 & $\mathrm{H}$ & 3.47867600 & -2.02289600 & -2.18507300 \\
\hline $\mathrm{H}$ & 3.02027400 & 0.59012800 & -5.38848400 & $\mathrm{H}$ & 2.63366600 & -2.31681900 & -3.71679600 \\
\hline $\mathrm{C}$ & 3.06545400 & -1.54090900 & -2.78861500 & $\mathrm{H}$ & 4.29448800 & -1.70536200 & -3.72533000 \\
\hline $\mathrm{H}$ & 3.16214400 & -1.81542100 & -1.73368300 & $\mathrm{Si}$ & 4.07493900 & -1.65639500 & 1.88271500 \\
\hline $\mathrm{H}$ & 2.39627000 & -2.26208300 & -3.27086500 & $\mathrm{C}$ & 4.97717500 & -0.13667000 & 2.54281700 \\
\hline $\mathrm{H}$ & 4.05359700 & -1.64260500 & -3.25457300 & $\mathrm{H}$ & 5.44850200 & 0.43622800 & 1.73622200 \\
\hline $\mathrm{Si}$ & 3.57854600 & -0.95116100 & 2.35072600 & $\mathrm{H}$ & 4.29812200 & 0.53434500 & 3.08050700 \\
\hline $\mathrm{C}$ & 5.10896500 & 0.10389000 & 2.03400300 & $\mathrm{H}$ & 5.76794500 & -0.43930900 & 3.24019900 \\
\hline $\mathrm{H}$ & 5.54183400 & -0.09333600 & 1.04676700 & $\mathrm{C}$ & 3.21892500 & -2.60766200 & 3.26771900 \\
\hline $\mathrm{H}$ & 4.88370700 & 1.17417800 & 2.09987600 & $\mathrm{H}$ & 2.66628400 & -3.47122700 & 2.88119900 \\
\hline $\mathrm{H}$ & 5.87928200 & -0.11840200 & 2.78254500 & $\mathrm{H}$ & 3.95927400 & -2.98087200 & 3.98584800 \\
\hline $\mathrm{C}$ & 2.79895700 & -0.56410100 & 4.02190200 & $\mathrm{H}$ & 2.51418800 & -1.97314700 & 3.81680000 \\
\hline $\mathrm{H}$ & 1.88971200 & -1.15174000 & 4.19045300 & $\mathrm{C}$ & 5.23673900 & -2.77071000 & 0.89909700 \\
\hline $\mathrm{H}$ & 3.50157300 & -0.80159000 & 4.82999100 & $\mathrm{H}$ & 4.70893700 & -3.63469900 & 0.47990500 \\
\hline $\mathrm{H}$ & 2.53805100 & 0.49627500 & 4.11059500 & $\mathrm{H}$ & 5.71329600 & -2.23073000 & 0.07307200 \\
\hline $\mathrm{C}$ & 3.95289400 & -2.79017800 & 2.16337500 & $\mathrm{H}$ & 6.03418800 & -3.15233500 & 1.54836000 \\
\hline $\mathrm{H}$ & 3.05359500 & -3.40159900 & 2.29690400 & $\mathrm{Ag}$ & -2.90013700 & 1.33854200 & -3.86984500 \\
\hline $\mathrm{H}$ & 4.37646900 & -3.02050700 & 1.17933100 & $\mathrm{~N}$ & -2.37183300 & 0.05743700 & 1.07100600 \\
\hline $\mathrm{H}$ & 4.68289700 & -3.10371600 & 2.91964800 & $\mathrm{~S}$ & -3.47910600 & -0.72776800 & 2.10669400 \\
\hline $\mathrm{Ag}$ & -3.11551700 & 1.42200900 & -4.01341400 & $\mathrm{O}$ & -3.22847300 & -2.17532300 & 2.09871900 \\
\hline $\mathrm{N}$ & -2.77591300 & 0.66114400 & 1.04851200 & $\mathrm{O}$ & -4.78305600 & -0.18927700 & 1.70950100 \\
\hline $\mathrm{S}$ & -3.86938900 & 0.53502500 & 2.32603400 & $\mathrm{C}$ & -3.01084000 & -0.09140200 & 3.70641300 \\
\hline $\mathrm{O}$ & -5.01275300 & 1.34600600 & 1.90838200 & $\mathrm{C}$ & -2.41276600 & -0.94398900 & 4.63411700 \\
\hline $\mathrm{O}$ & -3.07719600 & 0.80669500 & 3.52342100 & $\mathrm{C}$ & -3.29011400 & 1.24267400 & 4.02289700 \\
\hline $\mathrm{C}$ & -4.38991500 & -1.17572200 & 2.37996400 & $\mathrm{C}$ & -2.07953000 & -0.44414000 & 5.89380900 \\
\hline $\mathrm{C}$ & -5.50747500 & -1.58214600 & 1.64380100 & $\mathrm{H}$ & -2.22656000 & -1.98052300 & 4.37457100 \\
\hline $\mathrm{C}$ & -3.66652200 & -2.09266000 & 3.14990000 & $\mathrm{C}$ & -2.94920800 & 1.72108100 & 5.28282800 \\
\hline $\mathrm{C}$ & -5.88875600 & -2.92236000 & 1.67356000 & $\mathrm{H}$ & -3.77410300 & 1.88681400 & 3.29625800 \\
\hline $\mathrm{H}$ & -6.08709800 & -0.85102200 & 1.09004500 & $\mathrm{C}$ & -2.34100700 & 0.88803100 & 6.23850400 \\
\hline $\mathrm{C}$ & -4.06411000 & -3.42783100 & 3.16541100 & $\mathrm{H}$ & -1.61647900 & -1.10445600 & 6.62220900 \\
\hline $\mathrm{H}$ & -2.82964300 & -1.75446200 & 3.75181700 & $\mathrm{H}$ & -3.16437300 & 2.75613100 & 5.53614500 \\
\hline $\mathrm{C}$ & -5.17720200 & -3.86513600 & 2.43092200 & $\mathrm{C}$ & -2.00328500 & 1.41924600 & 7.61044100 \\
\hline $\mathrm{H}$ & -6.76379100 & -3.23942800 & 1.11196800 & $\mathrm{H}$ & -2.91327100 & 1.57492700 & 8.20374300 \\
\hline $\mathrm{H}$ & -3.51005000 & -4.14078100 & 3.77062100 & $\mathrm{H}$ & -1.36214300 & 0.72680100 & 8.16362100 \\
\hline $\mathrm{C}$ & -5.62297400 & -5.30591800 & 2.49089600 & $\mathrm{H}$ & -1.49006400 & 2.38556500 & 7.54944800 \\
\hline $\mathrm{H}$ & -4.77395900 & -5.98384900 & 2.62513100 & $\mathrm{C}$ & -6.41224400 & 2.09294800 & -8.12388100 \\
\hline $\mathrm{H}$ & -6.30379900 & -5.46621600 & 3.33698500 & $\mathrm{C}$ & -6.32608400 & 2.66831700 & -6.84122600 \\
\hline \multirow[t]{2}{*}{$\mathrm{H}$} & -6.15759700 & -5.60034100 & 1.58242400 & $\mathrm{C}$ & -7.27322300 & 3.61599500 & -6.40695500 \\
\hline & & & & $\mathrm{C}$ & -8.30495800 & 3.98271600 & -7.26458400 \\
\hline IN2 & & & & $\mathrm{C}$ & -8.39374600 & 3.41359600 & -8.53905200 \\
\hline $\mathrm{C}$ & -2.87417700 & 0.30421700 & -0.30284200 & $\mathrm{C}$ & -7.45132300 & 2.47284800 & -8.96679600 \\
\hline $\mathrm{H}$ & -3.41721500 & 1.25398700 & -0.34868500 & $\mathrm{H}$ & -5.67538200 & 1.36337300 & -8.44355500 \\
\hline
\end{tabular}




$\begin{array}{llll}\mathrm{H} & -7.19397200 & 4.04944700 & -5.41535800 \\ \mathrm{H} & -9.04054200 & 4.71155500 & -6.93976300 \\ \mathrm{H} & -9.20251100 & 3.70442100 & -9.20271200 \\ \mathrm{H} & -7.52743200 & 2.03543100 & -9.95709900 \\ \mathrm{C} & -5.26841500 & 2.28390600 & -5.96783100 \\ \mathrm{~N} & -4.40693500 & 1.97120500 & -5.25425600\end{array}$

\section{TS1}

$\mathrm{C}$

$\mathrm{H}$

$\mathrm{H}$

C

$\mathrm{H}$

$\mathrm{H}$

C

$\mathrm{C}$

$\mathrm{C}$

C

C

C

$\mathrm{C}$

$\mathrm{H}$

$\mathrm{H}$

$\mathrm{H}$

C

$\mathrm{H}$

$\mathrm{H}$

C

$\mathrm{H}$

$\mathrm{H}$

$\mathrm{H}$

$\mathrm{Si}$

C

$\mathrm{H}$

$\mathrm{H}$

$\mathrm{H}$

C

$\mathrm{H}$

$\mathrm{H}$

C

$\mathrm{H}$

H

$\mathrm{H}$

Ag

$\mathrm{N}$

$\mathrm{S}$

O

$\mathrm{O}$

C

C

C

C

$\mathrm{H}$

C

$\mathrm{H}$

$\mathrm{H}$

C

$\mathrm{H}$

$\mathrm{H}$

$\mathrm{H}$

$\begin{array}{lll}-3.96057900 & 0.56254700 & 0.95706800\end{array}$

$\begin{array}{llll}-4.57614700 & 1.45684000 & 1.09695100\end{array}$

$-4.63453400 \quad-0.30564400 \quad 0.85316500$

$\begin{array}{lll}-1.66752400 & -0.01597800 & 1.59684200\end{array}$

$\begin{array}{lll}-0.88203500 & 0.52583000 & 2.13058700\end{array}$

$\begin{array}{lll}-1.49189800 & -1.09426100 & 1.74404100\end{array}$

$\begin{array}{llll}-3.03666700 & 0.65804300 & -0.23326600\end{array}$

$\begin{array}{lll}-1.73112200 & 0.32112600 & 0.12969300\end{array}$

$\begin{array}{llll}-3.36523400 & 1.01838600 & -1.56677800\end{array}$

$\begin{array}{llll}-0.66271900 & 0.31271500 & -0.79411200\end{array}$

$\begin{array}{lll}-2.22851400 & 0.94084600 & -2.23577100\end{array}$

$\begin{array}{llll}0.62345300 & -0.05204500 & -0.31422100\end{array}$

$\begin{array}{llll}-0.91503300 & 0.67611100 & -2.18550800\end{array}$

$\begin{array}{lll}1.66702200 & -0.38255800 & 0.23053800\end{array}$

$\begin{array}{lll}0.37129800 & 0.77673700 & -3.64424500\end{array}$

$\begin{array}{lll}0.36492800 & 2.56757700 & -4.23701200\end{array}$

$\begin{array}{lll}1.02653800 & 2.67016100 & -5.10635200\end{array}$

$\begin{array}{lll}-0.62867800 & 2.92069600 & -4.52424400\end{array}$

$\begin{array}{llll}0.74849400 & 3.23190300 & -3.45385700\end{array}$

$\begin{array}{lll}-0.19256800 & -0.46880700 & -4.94438700\end{array}$

$-0.10722700 \quad-1.49176200 \quad-4.55959500$

$-1.22503900-0.31301000 \quad-5.26746400$

$\begin{array}{lll}0.45411000 & -0.39654900 & -5.82787400\end{array}$

$\begin{array}{llll}2.11177200 & 0.33189600 & -3.09142400\end{array}$

$\begin{array}{llll}2.50176000 & 1.00765000 & -2.32578600\end{array}$

$\begin{array}{lll}2.19636700 & -0.69220900 & -2.71892000\end{array}$

$\begin{array}{lll}2.75584500 & 0.42137200 & -3.97704200\end{array}$

$\begin{array}{lll}3.26048400 & -0.87388700 & 1.06346400\end{array}$

$\begin{array}{lll}4.32880500 & 0.67433000 & 1.20496000\end{array}$

$\begin{array}{lll}4.56387200 & 1.09631600 & 0.22140400\end{array}$

$\begin{array}{lll}3.83326200 & 1.45190600 & 1.79685800\end{array}$

$\begin{array}{lll}5.27860700 & 0.43516600 & 1.69888800\end{array}$

$\begin{array}{lll}2.81974400 & -1.56235600 & 2.76286200\end{array}$

$\begin{array}{lll}2.17662100 & -2.44630700 & 2.68550800\end{array}$

$\begin{array}{lll}3.72920700 & -1.86042100 & 3.29878700\end{array}$

$\begin{array}{lll}2.30137400 & -0.81883500 & 3.37857100\end{array}$

$\begin{array}{lll}4.08621100 & -2.18641100 & -0.01203700\end{array}$

$3.45499900 \quad-3.07590200-0.11742500$

$\begin{array}{lll}4.30848600 & -1.80764600 & -1.01604800\end{array}$

$\begin{array}{lll}5.03408300 & -2.50389800 & 0.43955200\end{array}$

$\begin{array}{llll}-5.23299300 & 1.62237200 & -2.38250500\end{array}$

$\begin{array}{lll}-2.99944400 & 0.41823600 & 2.05793400\end{array}$

$\begin{array}{lll}-3.53870900 & 0.03495300 & 3.60365600\end{array}$

$\begin{array}{lll}-4.78546000 & 0.78462800 & 3.76024600\end{array}$

$\begin{array}{lll}-2.37437200 & 0.21551600 & 4.46868300\end{array}$

$\begin{array}{lll}-3.94410900 & -1.70919100 & 3.58022700\end{array}$

$\begin{array}{lll}-5.24112200 & -2.11373600 & 3.24629700\end{array}$

$\begin{array}{lll}-2.95587500 & -2.65173300 & 3.88004300\end{array}$

$\begin{array}{lll}-5.53812900 & -3.47404000 & 3.20228100\end{array}$

$-6.00897400 \quad-1.36971000 \quad 3.06207600$

$\begin{array}{lll}-3.27327300 & -4.00824700 & 3.82953900\end{array}$

$\begin{array}{lll}-1.96647400 & -2.32292800 & 4.18016800\end{array}$

$\begin{array}{lll}-4.56344000 & -4.44189200 & 3.49144500\end{array}$

$\begin{array}{lll}-6.54821600 & -3.79066000 & 2.95427100\end{array}$

$\begin{array}{lll}-2.50933600 & -4.74258400 & 4.07162300\end{array}$

$\begin{array}{lll}-4.91045700 & -5.91113700 & 3.47886900\end{array}$

$\begin{array}{lll}-5.41583500 & -6.19936600 & 4.40974400\end{array}$

$\begin{array}{lll}-5.58824400 & -6.15680200 & 2.65431500\end{array}$

$\begin{array}{lll}-4.01644100 & -6.53491800 & 3.38480700\end{array}$ $\begin{array}{lll}-4.07574800 & 1.31544900 & -7.78808600\end{array}$

$\begin{array}{lll}-3.63549900 & 2.29226500 & -6.87490600\end{array}$

$\begin{array}{lll}-3.57344300 & 3.64884800 & -7.24734600\end{array}$

$\begin{array}{llll}-3.95521000 & 4.01940600 & -8.53321100\end{array}$

$\begin{array}{lll}-4.39419800 & 3.05081100 & -9.44118900\end{array}$

$\begin{array}{llll}-4.45360400 & 1.70391900 & -9.06966800\end{array}$

$\begin{array}{lll}-4.11339400 & 0.27225300 & -7.49129900\end{array}$

$\begin{array}{llll}-3.22670900 & 4.39190100 & -6.53617200\end{array}$

$\begin{array}{llll}-3.90865400 & 5.06287300 & -8.82819600\end{array}$

$\begin{array}{llll}-4.68987900 & 3.34646100 & -10.44333300\end{array}$

$\begin{array}{lll}-4.79258000 & 0.95672100 & -9.78026400\end{array}$

$\begin{array}{llll}-3.24015900 & 1.90271900 & -5.55792300\end{array}$

$\begin{array}{llll}-2.91379600 & 1.58647900 & -4.48473800\end{array}$

IN3

C

$\mathrm{H}$

$\mathrm{H}$

C

$\mathrm{H}$

$\mathrm{H}$

C

C

C

C

C

C

C

C

$\mathrm{Si}$

C

$\mathrm{H}$

$\mathrm{H}$

$\mathrm{H}$

C

$\mathrm{H}$

$\mathrm{H}$

$\mathrm{H}$

C

$\mathrm{H}$

$\mathrm{H}$

$\mathrm{H}$

$\mathrm{Si}$

C

$\mathrm{H}$

$\mathrm{H}$

$\mathrm{H}$

C

$\mathrm{H}$

$\mathrm{H}$

$\mathrm{H}$

C

$\mathrm{H}$

$\mathrm{H}$

$\mathrm{H}$

$\mathrm{Ag}$

$\mathrm{N}$

$\mathrm{S}$

O

O

C

C

C

C

$\mathrm{H}$

C

$\mathrm{H}$ $\begin{array}{lll}-4.02860200 & 0.61943000 & 0.78945100\end{array}$

$\begin{array}{lll}-4.65785100 & 1.50359000 & 0.93429700\end{array}$

$\begin{array}{lll}-4.68985100 & -0.26055600 & 0.70443200\end{array}$

$\begin{array}{lll}-1.73118300 & 0.06828900 & 1.40869100\end{array}$

$\begin{array}{lll}-0.93677600 & 0.61457400 & 1.92514000\end{array}$

$\begin{array}{lll}-1.54446800 & -1.00872100 & 1.55826100\end{array}$

$\begin{array}{lll}-3.12294200 & 0.72056600 & -0.41598000\end{array}$

$\begin{array}{lll}-1.81549600 & 0.39142100 & -0.05799900\end{array}$

$\begin{array}{lll}-3.45125500 & 1.06823300 & -1.72043700\end{array}$

$\begin{array}{lll}-0.75704500 & 0.37565700 & -0.99131400\end{array}$

$\begin{array}{lll}-2.37221900 & 1.05338500 & -2.63266100\end{array}$

$\begin{array}{llll}0.52210000 & 0.00554300 & -0.47548100\end{array}$

$\begin{array}{lll}-1.01720600 & 0.72026300 & -2.34357700\end{array}$

$\begin{array}{lll}1.54077300 & -0.32733700 & 0.11150300\end{array}$

$\begin{array}{lll}0.32954900 & 0.76733600 & -3.73627900\end{array}$

$\begin{array}{llll}0.41362500 & 2.53945800 & -4.40849600\end{array}$

$\begin{array}{lll}1.19542000 & 2.59597800 & -5.17602900\end{array}$

$\begin{array}{lll}-0.51140300 & 2.90917400 & -4.86265400\end{array}$

$\begin{array}{llll}0.69260400 & 3.23802000 & -3.61084200\end{array}$

$\begin{array}{lll}-0.17172800 & -0.47560000 & -5.07940600\end{array}$

$-0.20473500 \quad-1.49147400 \quad-4.66827700$

$\begin{array}{lll}-1.13830500 & -0.28065200 & -5.55539300\end{array}$

$\begin{array}{lll}0.58607200 & -0.47237600 & -5.87265500\end{array}$

$\begin{array}{lll}2.06705800 & 0.30800700 & -3.17911000\end{array}$

$\begin{array}{llll}2.46242800 & 0.98817000 & -2.42048200\end{array}$

$2.13485100 \quad-0.71011500 \quad-2.78782600$

$\begin{array}{lll}2.71511000 & 0.37453000 & -4.06349600\end{array}$

$\begin{array}{lll}3.10543700 & -0.82185700 & 0.98826700\end{array}$

$\begin{array}{lll}4.20702800 & 0.70582400 & 1.10718000\end{array}$

$\begin{array}{lll}4.46817800 & 1.09510600 & 0.11660800\end{array}$

$\begin{array}{lll}3.71795300 & 1.51021500 & 1.66791600\end{array}$

$\begin{array}{llll}5.14269200 & 0.46087200 & 1.62471500\end{array}$

$\begin{array}{llll}2.62643300 & -1.44983400 & 2.70173100\end{array}$

$\begin{array}{lll}1.97146700 & -2.32649000 & 2.64084000\end{array}$

$\begin{array}{lll}3.52126300 & -1.74359600 & 3.26403500\end{array}$

$\begin{array}{lll}2.10767400 & -0.67973300 & 3.28374300\end{array}$

$\begin{array}{lll}3.93114800 & -2.18389100 & -0.02523800\end{array}$

$3.28344700 \quad-3.06280600-0.11991500$

$\begin{array}{lll}4.18507600 & -1.84044100 & -1.03450600\end{array}$

$\begin{array}{llll}4.86094300 & -2.50736400 & 0.45854700\end{array}$

$\begin{array}{lll}-5.45618900 & 1.60060400 & -2.16185400\end{array}$

$\begin{array}{lll}-3.05669500 & 0.49861200 & 1.88450300\end{array}$

$\begin{array}{lll}-3.57295600 & 0.12264300 & 3.43797200\end{array}$

$\begin{array}{lll}-4.83029500 & 0.85289100 & 3.60317800\end{array}$

$\begin{array}{lll}-2.40386800 & 0.32888000 & 4.29121800\end{array}$

$\begin{array}{lll}-3.94944000 & -1.62860300 & 3.43553600\end{array}$

$\begin{array}{lll}-5.24319800 & -2.05858400 & 3.12564800\end{array}$

$\begin{array}{lll}-2.93960000 & -2.55185500 & 3.72668800\end{array}$

$\begin{array}{lll}-5.51670900 & -3.42520500 & 3.09665900\end{array}$

$\begin{array}{lll}-6.02680900 & -1.32979800 & 2.94699900\end{array}$

$\begin{array}{lll}-3.23293600 & -3.91334400 & 3.69124000\end{array}$

$\begin{array}{lll}-1.95162300 & -2.20300200 & 4.00830000\end{array}$ 


$\begin{array}{lrrr}\mathrm{C} & -4.52120900 & -4.37277700 & 3.37706200 \\ \mathrm{H} & -6.52459300 & -3.76172000 & 2.86701800 \\ \mathrm{H} & -2.45174900 & -4.63207100 & 3.92581100 \\ \mathrm{C} & -4.83629400 & -5.84922100 & 3.38413600 \\ \mathrm{H} & -5.09205800 & -6.18751300 & 4.39670700 \\ \mathrm{H} & -5.68825600 & -6.08219300 & 2.73774900 \\ \mathrm{H} & -3.97989800 & -6.44448000 & 3.05077500 \\ \mathrm{C} & -3.82311200 & 1.10551500 & -7.23319100 \\ \mathrm{C} & -3.40144100 & 2.09618900 & -6.31874600 \\ \mathrm{C} & -3.35179000 & 3.45780900 & -6.69192100 \\ \mathrm{C} & -3.72625000 & 3.81682000 & -7.98104400 \\ \mathrm{C} & -4.14439700 & 2.83653700 & -8.88849000 \\ \mathrm{C} & -4.19284200 & 1.48784800 & -8.51688900 \\ \mathrm{H} & -3.85253800 & 0.06349600 & -6.93191600 \\ \mathrm{H} & -3.02250900 & 4.20622400 & -5.97851500 \\ \mathrm{H} & -3.69120000 & 4.85906200 & -8.28098200 \\ \mathrm{H} & -4.43403500 & 3.12588300 & -9.89422500 \\ \mathrm{H} & -4.51704400 & 0.73673500 & -9.22978400 \\ \mathrm{C} & -3.01611900 & 1.71858100 & -5.01072500 \\ \mathrm{~N} & -2.68626700 & 1.41017700 & -3.94136200\end{array}$

\section{TS2}

$\begin{array}{lll}-3.65572900 & 1.88939200 & 1.89153300\end{array}$

$\begin{array}{lll}-3.50078600 & 2.97467300 & 1.82691800\end{array}$

$\begin{array}{lll}-4.71553800 & 1.73374900 & 2.10230900\end{array}$

$\begin{array}{lll}-1.48677500 & 0.99580900 & 2.36309500\end{array}$

$\begin{array}{lll}-0.79456200 & 1.83391000 & 2.51838900\end{array}$

$\begin{array}{lll}-1.04825300 & 0.12487600 & 2.85477200\end{array}$

$\begin{array}{lll}-3.12130400 & 1.20915700 & 0.64650200\end{array}$

$\begin{array}{lll}-1.80221800 & 0.78958200 & 0.89395200\end{array}$

$\begin{array}{lll}-3.71602700 & 0.83583000 & -0.54924200\end{array}$

$\begin{array}{lll}-0.99202200 & 0.19473300 & -0.09012500\end{array}$

$\begin{array}{lll}-2.85646800 & 0.29832200 & -1.56338800\end{array}$

$\begin{array}{lll}0.32023000 & -0.18203400 & 0.32033600\end{array}$

$\begin{array}{lll}-1.50500600 & -0.01190200 & -1.41718400\end{array}$

$\begin{array}{lll}1.41178000 & -0.46426300 & 0.79362300\end{array}$

$\begin{array}{lll}-0.52797300 & -0.63870500 & -2.96448800\end{array}$

$\begin{array}{llll}-0.54654000 & 0.76248400 & -4.23526400\end{array}$

$\begin{array}{lll}-0.00527500 & 0.45415800 & -5.13818600\end{array}$

$\begin{array}{lll}-1.56395500 & 1.03446800 & -4.53484800\end{array}$

$\begin{array}{lll}-0.05331100 & 1.66192200 & -3.84837400\end{array}$

$\begin{array}{lll}-1.43689300 & -2.16460600 & -3.61624400\end{array}$

$\begin{array}{lll}-1.44116900 & -2.97294900 & -2.87500300\end{array}$

$\begin{array}{lll}-2.47421400 & -1.94429800 & -3.88865300\end{array}$

$\begin{array}{lll}-0.93081200 & -2.54602400 & -4.51167200\end{array}$

$1.26083600 \quad-1.10596300 \quad-2.60017000$

$\begin{array}{llll}1.85013000 & -0.26466800 & -2.22442800\end{array}$

$\begin{array}{lll}1.34574900 & -1.92534500 & -1.88069300\end{array}$

$\begin{array}{lll}1.71453800 & -1.43909000 & -3.54304100\end{array}$

$\begin{array}{lll}3.09452800 & -0.88812000 & 1.46829500\end{array}$

$\begin{array}{lll}4.35849500 & 0.17060700 & 0.55077400\end{array}$

$\begin{array}{lll}4.33846500 & -0.01993700 & -0.52809500\end{array}$

$\begin{array}{lll}4.17740700 & 1.23967600 & 0.70885100\end{array}$

$\begin{array}{lll}5.37114200 & -0.05166600 & 0.90905800\end{array}$

$\begin{array}{lll}3.09162300 & -0.49798200 & 3.31382000\end{array}$

$\begin{array}{lll}2.33826200 & -1.08374600 & 3.85265400\end{array}$

$\begin{array}{lll}4.06904100 & -0.73266500 & 3.75284600\end{array}$

$\begin{array}{lll}2.89195300 & 0.56308600 & 3.50148200\end{array}$

$\begin{array}{lll}3.38883000 & -2.72736900 & 1.16159500\end{array}$

$\begin{array}{lll}2.64042800 & -3.34584200 & 1.66980700\end{array}$

$\begin{array}{lll}3.35881600 & -2.96848700 & 0.09304000\end{array}$

$\begin{array}{lll}4.37511400 & -3.02194400 & 1.54062600\end{array}$

$\begin{array}{lll}-5.49491800 & -0.33672800 & -0.00564200\end{array}$

$\begin{array}{lll}-2.79863900 & 1.34639800 & 2.96875100\end{array}$

$\begin{array}{lll}-3.54419400 & 0.23972200 & 4.01698700\end{array}$

$\begin{array}{lll}-4.63449000 & 0.97363600 & 4.65312500\end{array}$

$\begin{array}{lll}-2.46393300 & -0.41499900 & 4.74952800\end{array}$ $\begin{array}{lll}-4.35177300 & -1.03011700 & 3.00194400\end{array}$ $\begin{array}{lll}-5.69720300 & -0.87561400 & 2.64917400\end{array}$

$\begin{array}{lll}-3.63439700 & -2.14852700 & 2.56632200\end{array}$ $\begin{array}{lll}-6.31153200 & -1.82996600 & 1.80581100\end{array}$ $\begin{array}{lll}-6.27429200 & -0.05738100 & 3.06891000\end{array}$ $\begin{array}{lll}-4.24865400 & -3.09494600 & 1.74484600\end{array}$ $\begin{array}{lll}-2.60651100 & -2.28620400 & 2.88616900\end{array}$ $\begin{array}{lll}-5.59068800 & -2.96290500 & 1.34823800\end{array}$ $\begin{array}{lll}-7.38444400 & -1.77162600 & 1.62835700\end{array}$ $-3.68497400 \quad-3.96742500 \quad 1.42583400$ $\begin{array}{lll}-6.26936900 & -4.04313500 & 0.54223700\end{array}$ $\begin{array}{llll}-5.61301000 & -4.43395000 & -0.24173800\end{array}$ $\begin{array}{lll}-6.53320300 & -4.88640900 & 1.19299700\end{array}$ $\begin{array}{lll}-7.19351500 & -3.68763500 & 0.07712700\end{array}$ $\begin{array}{lll}-6.86531400 & 1.97967700 & -2.52124800\end{array}$ $\begin{array}{lll}-5.65153300 & 1.70418500 & -3.18327000\end{array}$ $\begin{array}{lll}-5.42674000 & 2.20039400 & -4.49158000\end{array}$ $\begin{array}{lll}-6.42464600 & 2.93000200 & -5.12397400\end{array}$ $\begin{array}{lll}-7.63164700 & 3.19000200 & -4.46368700\end{array}$ $\begin{array}{lll}-7.84983400 & 2.71909300 & -3.16350200\end{array}$ $\begin{array}{lll}-7.00917000 & 1.63020200 & -1.50263600\end{array}$ $\begin{array}{lll}-4.48540100 & 1.99100000 & -4.98839800\end{array}$ $\begin{array}{lll}-6.26166700 & 3.30435400 & -6.12970500\end{array}$ $\begin{array}{lll}-8.40243700 & 3.77085300 & -4.96137600\end{array}$ $\begin{array}{lll}-8.78320000 & 2.93768100 & -2.65473300\end{array}$ $\begin{array}{lll}-4.63168900 & 0.91682000 & -2.58576700\end{array}$ $\begin{array}{llll}-3.58363300 & 0.29022100 & -2.78375800\end{array}$

IN4

C $\mathrm{H}$

$\mathrm{H}$

C

$\mathrm{H}$

$\mathrm{H}$

C

C

\begin{abstract}
$\begin{array}{lll}-4.04307300 & 2.01611600 & 1.31738700\end{array}$ $\begin{array}{lll}-3.84616900 & 3.08325000 & 1.15239300\end{array}$ $\begin{array}{lll}-5.12097700 & 1.89340200 & 1.44166200\end{array}$ $\begin{array}{lll}-1.92736000 & 1.20170700 & 2.07836200\end{array}$ $\begin{array}{lll}-1.28244900 & 2.08492300 & 2.16794200\end{array}$ $\begin{array}{lll}-1.51301900 & 0.42424300 & 2.72258400\end{array}$ $\begin{array}{lll}-3.41421200 & 1.18410400 & 0.21572100\end{array}$ $\begin{array}{llll}-2.12459700 & 0.77963300 & 0.63637900\end{array}$ $\begin{array}{lll}-3.84339400 & 0.68639500 & -1.02076900\end{array}$ $\begin{array}{llll}-1.22809000 & 0.06786700 & -0.18339000\end{array}$ $\begin{array}{llll}-2.87507700 & 0.03409300 & -1.85953600\end{array}$ $\begin{array}{rrr}0.02925500 & -0.25620400 & 0.39298800\end{array}$ $\begin{array}{lll}-1.57730300 & -0.30023800 & -1.54981800\end{array}$ $\begin{array}{lll}1.07871200 & -0.49820700 & 0.97419400\end{array}$ $\begin{array}{lll}-0.45257700 & -1.02970400 & -2.93766700\end{array}$ $\begin{array}{llll}0.01446500 & 0.42051800 & -4.05478600\end{array}$ $\begin{array}{llll}0.62739700 & 0.07372300 & -4.89582700\end{array}$ $\begin{array}{llll}-0.87717100 & 0.90419000 & -4.46923400\end{array}$ $\begin{array}{llll}0.59346200 & 1.17899700 & -3.51495300\end{array}$ $\begin{array}{lll}-1.50855600 & -2.28971200 & -3.86671400\end{array}$ $-1.78093300 \quad-3.13753400 \quad-3.22605000$ $-2.42947700 \quad-1.83855600-4.25002700$ $\begin{array}{lll}-0.95008000 & -2.69298700 & -4.72034400\end{array}$ $\begin{array}{lll}1.09469100 & -1.87838800 & -2.27246900\end{array}$ $\begin{array}{lll}1.80535700 & -1.17149300 & -1.83561600\end{array}$ $\begin{array}{llll}0.86655700 & -2.63993200 & -1.51922300\end{array}$ $\begin{array}{lll}1.59363900 & -2.38132100 & -3.11090100\end{array}$ $\begin{array}{lll}2.69132300 & -0.90247700 & 1.81993800\end{array}$ $\begin{array}{lll}4.06743600 & -0.06892900 & 0.83494300\end{array}$ $\begin{array}{lll}4.10980900 & -0.43925400 & -0.19543400\end{array}$ $\begin{array}{llll}3.93655300 & 1.01831700 & 0.79871800\end{array}$ $\begin{array}{rrr}5.03969400 & -0.27130600 & 1.30075400\end{array}$ $\begin{array}{lll}2.60179900 & -0.22597500 & 3.57851200\end{array}$ $\begin{array}{lll}1.77273600 & -0.67002500 & 4.14112400\end{array}$ $\begin{array}{lll}3.52814200 & -0.45365000 & 4.11993700\end{array}$ $\begin{array}{lll}2.47350700 & 0.86239900 & 3.58681200\end{array}$ $\begin{array}{lll}2.87041600 & -2.78001500 & 1.81290100\end{array}$ $\begin{array}{lll}2.06030700 & -3.26425700 & 2.36954500\end{array}$
\end{abstract}




$\begin{array}{lccc}\mathrm{H} & 2.86711400 & -3.17978800 & 0.79283800 \\ \mathrm{H} & 3.81766700 & -3.07172300 & 2.28274400 \\ \mathrm{Ag} & -4.78063400 & -1.03976500 & 0.37345700 \\ \mathrm{~N} & -3.29977300 & 1.58967900 & 2.53364400 \\ \mathrm{~S} & -4.10389500 & 0.40524800 & 3.47273500 \\ \mathrm{O} & -3.07997900 & -0.23867800 & 4.29115800 \\ \mathrm{O} & -4.96456200 & -0.48285400 & 2.61587500 \\ \mathrm{C} & -5.22805600 & 1.38541700 & 4.43892400 \\ \mathrm{C} & -4.72495900 & 2.46331400 & 5.17829700 \\ \mathrm{C} & -6.57001600 & 1.00793600 & 4.52311900 \\ \mathrm{C} & -5.59370600 & 3.17604000 & 5.99607900 \\ \mathrm{H} & -3.67937300 & 2.74229400 & 5.10510900 \\ \mathrm{C} & -7.42088200 & 1.73776300 & 5.35211300 \\ \mathrm{H} & -6.93792500 & 0.16129900 & 3.95494100 \\ \mathrm{C} & -6.95176700 & 2.82600400 & 6.10056900 \\ \mathrm{H} & -5.21289700 & 4.01818700 & 6.56751500 \\ \mathrm{H} & -8.46671100 & 1.45192500 & 5.42065000 \\ \mathrm{C} & -7.87115000 & 3.59669900 & 7.01461900 \\ \mathrm{H} & -7.66921200 & 3.34895000 & 8.06444900 \\ \mathrm{H} & -7.72787000 & 4.67717100 & 6.90543200 \\ \mathrm{H} & -8.92199900 & 3.36811800 & 6.81630300 \\ \mathrm{C} & -6.59809700 & 2.18651100 & -1.86136800 \\ \mathrm{C} & -5.76206100 & 1.54980000 & -2.79795300 \\ \mathrm{C} & -6.10811000 & 1.55956400 & -4.16603200 \\ \mathrm{C} & -7.27373500 & 2.19170600 & -4.57792700 \\ \mathrm{C} & -8.10206900 & 2.82092400 & -3.63884700 \\ \mathrm{C} & -7.76476600 & 2.81829800 & -2.28220800 \\ \mathrm{H} & -6.32596700 & 2.18912300 & -0.80892600 \\ \mathrm{H} & -5.45349300 & 1.06783000 & -4.87839300 \\ \mathrm{H} & -7.54239200 & 2.19958800 & -5.62978600 \\ \mathrm{H} & -9.01096000 & 3.31626100 & -3.96764000 \\ \mathrm{H} & -8.40834800 & 3.31103900 & -1.55990000 \\ \mathrm{C} & -4.56005000 & 0.88058900 & -2.37230600 \\ \mathrm{~N} & -3.67208600 & 0.20952700 & -3.07008000\end{array}$

\section{IN5}

C



$\begin{array}{lll}4.17628700 & 2.30552200 & 3.59330500\end{array}$ $\begin{array}{lll}2.94059100 & -0.76225600 & 3.14921400\end{array}$ $\begin{array}{lll}2.83254700 & -1.69790300 & 2.58893300\end{array}$ $\begin{array}{lll}3.83015900 & -0.85560100 & 3.78422700\end{array}$ $\begin{array}{lll}2.07049500 & -0.66120700 & 3.80791800\end{array}$ $\begin{array}{lll}4.57307300 & 0.49687000 & 0.82119900\end{array}$ $\begin{array}{lll}4.47398500 & -0.41807900 & 0.22634900\end{array}$ $\begin{array}{llll}4.66699400 & 1.34085000 & 0.12833800\end{array}$ $\begin{array}{llll}5.50979200 & 0.42635000 & 1.38768500\end{array}$ $\begin{array}{lll}-5.07343200 & -0.00472500 & -2.66784600\end{array}$ $\begin{array}{lll}-2.61422200 & -1.96090200 & 1.02050200\end{array}$ $\begin{array}{lll}-2.63140600 & -3.58580200 & 1.51209100\end{array}$ $\begin{array}{lll}-1.51000800 & -4.31721700 & 0.90450300\end{array}$ $\begin{array}{lll}-4.01295600 & -4.02354700 & 1.28867200\end{array}$ $\begin{array}{lll}-2.31025500 & -3.44358500 & 3.26418600\end{array}$ $\begin{array}{lll}-1.11040800 & -3.92848000 & 3.78289600\end{array}$ $\begin{array}{lll}-3.28457000 & -2.88412300 & 4.09791000\end{array}$ $\begin{array}{lll}-0.88194100 & -3.83860000 & 5.15710200\end{array}$ $\begin{array}{lll}-0.37763000 & -4.37527900 & 3.11975600\end{array}$ $\begin{array}{lll}-3.03767900 & -2.80234600 & 5.46366800\end{array}$ $\begin{array}{lll}-4.21974700 & -2.52658900 & 3.67979200\end{array}$ $\begin{array}{lll}-1.83539000 & -3.27807700 & 6.01595800\end{array}$ $\begin{array}{llll}0.05083600 & -4.21686000 & 5.56705600\end{array}$ $\begin{array}{lll}-3.79072500 & -2.36839300 & 6.11687900\end{array}$ $\begin{array}{lll}-1.59521300 & -3.20183200 & 7.50458700\end{array}$ $\begin{array}{lll}-2.23701100 & -3.91157900 & 8.04185400\end{array}$ $\begin{array}{lll}-0.55717000 & -3.43736600 & 7.75694200\end{array}$ $\begin{array}{lll}-1.82284800 & -2.20334000 & 7.89509500\end{array}$ $\begin{array}{llll}-4.53518500 & 4.93703900 & -4.59026300\end{array}$ $\begin{array}{lll}-3.72433200 & 3.82963700 & -4.91968000\end{array}$ $\begin{array}{lll}-3.40813400 & 3.53547700 & -6.26355400\end{array}$ $\begin{array}{llll}-3.90612400 & 4.35573500 & -7.26914100\end{array}$ $\begin{array}{llll}-4.70879000 & 5.45497700 & -6.94428200\end{array}$ $\begin{array}{lll}-5.02258100 & 5.74416300 & -5.61156700\end{array}$ $\begin{array}{lll}-4.76818600 & 5.14996800 & -3.55211100\end{array}$ $\begin{array}{lll}-2.78249000 & 2.68104000 & -6.49990300\end{array}$ $\begin{array}{llll}-3.66790700 & 4.14118600 & -8.30589600\end{array}$ $\begin{array}{llll}-5.09198700 & 6.09192800 & -7.73591100\end{array}$ $\begin{array}{lll}-5.64503700 & 6.59982400 & -5.37065600\end{array}$ $\begin{array}{llll}-3.21206000 & 3.00611600 & -3.88658000\end{array}$ $\begin{array}{lll}-2.77439400 & 2.34208500 & -3.04187300\end{array}$ $\begin{array}{lll}-9.17521700 & -1.66378600 & -4.34445600\end{array}$ $\begin{array}{lll}-10.39738000 & -1.21290000 & -3.81064500\end{array}$ $-11.58339600 \quad-1.79450000 \quad-4.24706100$ $\begin{array}{llll}-11.55456000 & -2.81388100 & -5.20394400\end{array}$ $-10.33883600 \quad-3.26002700 \quad-5.73159600$ $\begin{array}{lll}-9.14261500 & -2.69049400 & -5.30725300\end{array}$ $\begin{array}{lll}-10.40640200 & -0.42321600 & -3.06645600\end{array}$ $\begin{array}{llll}-12.53043900 & -1.45485800 & -3.83991600\end{array}$ $-12.48421200 \quad-3.26435500 \quad-5.53865500$ $\begin{array}{lll}-10.32355100 & -4.05349400 & -6.47196600\end{array}$ $\begin{array}{lll}-8.19264500 & -3.03049300 & -5.70654900\end{array}$ $\begin{array}{lll}-7.95283000 & -1.07947400 & -3.89856200\end{array}$ $\begin{array}{lll}-6.95648000 & -0.60460100 & -3.53694500\end{array}$

$\begin{array}{ccc}-4.34496200 & -2.37636600 & 0.55031100 \\ -5.40939200 & -2.18067000 & 0.72773300 \\ -4.21941900 & -3.45435700 & 0.36461900 \\ -2.23003900 & -1.38381900 & 1.22323000 \\ -1.91492200 & -0.53524400 & 1.83569200 \\ -1.42246400 & -2.13012600 & 1.26231800 \\ -3.77241900 & -1.56615100 & -0.58933500 \\ -2.54980100 & -1.01795700 & -0.19969400 \\ -4.28730800 & -1.36358700 & -1.86412400 \\ -1.75790700 & -0.27867700 & -1.09433900 \\ -3.49192300 & -0.58399300 & -2.74047400\end{array}$




\section{IN6}

C

$\mathrm{H}$

$\mathrm{H}$

C

$\mathrm{H}$

$\mathrm{H}$

C

C

C

C

C

C

C

C

$\mathrm{Si}$

$\mathrm{H}$

$\mathrm{H}$

C

$\mathrm{H}$

$\mathrm{H}$

C

$\mathrm{H}$

$\mathrm{H}$

$\mathrm{Si}$

C

$\mathrm{H}$

$\mathrm{H}$

$\mathrm{H}$

C

$\mathrm{H}$

$\mathrm{H}$

$\mathrm{H}$

$\mathrm{H}$

$\mathrm{H}$

$\mathrm{H}$

Ag

$\mathrm{N}$

C

C

C

C

$\mathrm{H}$

$\mathrm{H}$

$\mathrm{H}$

$\mathrm{H}$

C

$\mathrm{N}$

C

C
$-3.69699300 \quad-4.85555700 \quad-2.22114800$

$-4.73221900 \quad-4.56466500 \quad-1.99370700$

$\begin{array}{lll}-3.73603200 & -5.74345900 & -2.85618100\end{array}$

$-2.25388200 \quad-3.92793100 \quad-0.53482800$

$\begin{array}{lll}-2.84234900 & -3.38377100 & 0.21525000\end{array}$

$\begin{array}{lll}-1.29134900 & -4.17494500 & -0.08028300\end{array}$

$-2.92439500 \quad-3.69168700 \quad-2.80742100$

$\begin{array}{lll}-2.11689000 & -3.13886400 & -1.81593400\end{array}$

$-2.96638000 \quad-3.15964800 \quad-4.09041800$

$\begin{array}{lll}-1.36312600 & -1.97252000 & -2.04291000\end{array}$

$-2.15870400 \quad-2.02054300 \quad-4.33075900$

$\begin{array}{lll}-0.62134300 & -1.48496400 & -0.92223700\end{array}$

$\begin{array}{lll}-1.39054900 & -1.36094800 & -3.32817700\end{array}$

$\begin{array}{lll}-0.04487400 & -1.18855600 & 0.11393100\end{array}$

$\begin{array}{lll}-0.52211600 & 0.30722800 & -3.77362800\end{array}$

$\begin{array}{lll}-1.87952100 & 1.54943300 & -4.22241600\end{array}$

$\begin{array}{lll}-1.43318900 & 2.49119100 & -4.56548800\end{array}$

$\begin{array}{lll}-2.54475700 & 1.18032700 & -5.00808800\end{array}$

$\begin{array}{lll}-2.49359800 & 1.77957600 & -3.34360600\end{array}$

$\begin{array}{llll}0.69159000 & 0.05182300 & -5.21412500\end{array}$

$\begin{array}{lll}1.36086700 & -0.79813500 & -5.02972600\end{array}$

$\begin{array}{lll}0.20483200 & -0.09523100 & -6.18274500\end{array}$

$\begin{array}{lll}1.32583000 & 0.94256600 & -5.30313100\end{array}$

$\begin{array}{llll}0.49091200 & 1.06369700 & -2.37138200\end{array}$

$\begin{array}{lll}-0.11250300 & 1.30386700 & -1.49221600\end{array}$

$\begin{array}{lll}1.31822400 & 0.42590500 & -2.04792800\end{array}$

$\begin{array}{llll}0.91749200 & 2.00116000 & -2.75353300\end{array}$

$\begin{array}{lll}0.83746600 & -0.67869500 & 1.66183700\end{array}$

$\begin{array}{lll}0.84157600 & -2.15265400 & 2.84423000\end{array}$

$\begin{array}{lll}-0.17724100 & -2.45951900 & 3.10723700\end{array}$

$\begin{array}{lll}1.35248900 & -3.01766400 & 2.40596600\end{array}$

$\begin{array}{lll}1.36158900 & -1.89626300 & 3.77547600\end{array}$

$\begin{array}{lll}2.60205000 & -0.18766600 & 1.20250200\end{array}$

$\begin{array}{llll}2.61300900 & 0.64381700 & 0.48880100\end{array}$

$\begin{array}{lll}3.15648000 & 0.13131700 & 2.09364300\end{array}$

$\begin{array}{lll}3.14779500 & -1.02503100 & 0.75289500\end{array}$

$\begin{array}{lll}-0.09468100 & 0.77893700 & 2.41794400\end{array}$

$\begin{array}{lll}-0.12504300 & 1.63519200 & 1.73472200\end{array}$

$\begin{array}{lll}-1.12788900 & 0.50969300 & 2.66469800\end{array}$

$\begin{array}{lll}0.39423700 & 1.10844700 & 3.34303000\end{array}$

$\begin{array}{lll}-4.40969300 & -3.88775300 & -5.46410900\end{array}$

$\begin{array}{lll}-2.99788000 & -5.15424800 & -0.94158000\end{array}$

$\begin{array}{lll}-2.41394300 & -0.16005300 & -8.21286400\end{array}$

$\begin{array}{lll}-1.89357300 & -1.45704600 & -8.04805200\end{array}$

$\begin{array}{lll}-1.49545500 & -2.19414100 & -9.17575700\end{array}$

$\begin{array}{llll}-1.61171800 & -1.64081200 & -10.44892500\end{array}$

$-2.12232900-0.35067600-10.60659000$

$\begin{array}{llll}-2.52259000 & 0.38648800 & -9.48656200\end{array}$

$\begin{array}{lll}-2.72314800 & 0.40238500 & -7.33905900\end{array}$

$\begin{array}{lll}-1.10326600 & -3.20047000 & -9.06464400\end{array}$

$\begin{array}{llll}-1.30482100 & -2.21672200 & -11.31648700\end{array}$

$\begin{array}{lll}-2.21088200 & 0.07979900 & -11.59959800\end{array}$

$\begin{array}{llll}-2.92118700 & 1.38892400 & -9.60849700\end{array}$

$\begin{array}{lll}-1.78444100 & -2.00685700 & -6.69067600\end{array}$

$\begin{array}{llll}-2.16314400 & -1.44763200 & -5.61936900\end{array}$

$\begin{array}{lll}0.43425100 & -5.13739500 & -5.73635300\end{array}$

$\begin{array}{lll}0.26771700 & -5.65614500 & -4.43041300\end{array}$

$1.09727600 \quad-6.68929800 \quad-4.01300300$ 


$\begin{array}{lrrr}\mathrm{C} & 2.07799400 & -7.19230100 & -4.87459700 \\ \mathrm{C} & 2.24532100 & -6.67027300 & -6.16591500 \\ \mathrm{C} & 1.42987400 & -5.64154900 & -6.61052400 \\ \mathrm{H} & -0.48607000 & -5.25838400 & -3.76079400 \\ \mathrm{H} & 0.96054700 & -7.08721800 & -3.01376500 \\ \mathrm{H} & 2.72231600 & -7.99980500 & -4.53948900 \\ \mathrm{H} & 3.01240400 & -7.06984800 & -6.82125900 \\ \mathrm{H} & 1.54520800 & -5.22660500 & -7.60637100 \\ \mathrm{C} & -0.40608500 & -4.10665000 & -6.18022100 \\ \mathrm{~N} & -1.13147000 & -3.30278400 & -6.61646400 \\ \mathrm{~S} & -2.06124400 & -6.58367300 & -0.97383500 \\ \mathrm{O} & -0.67340800 & -6.30275800 & -1.40647600 \\ \mathrm{O} & -2.85418600 & -7.55910900 & -1.73038300 \\ \mathrm{C} & -2.01078700 & -7.02458900 & 0.75271600 \\ \mathrm{C} & -3.20077800 & -7.36626100 & 1.40558400 \\ \mathrm{C} & -0.78362300 & -7.07801000 & 1.41225700 \\ \mathrm{C} & -3.14821400 & -7.75321100 & 2.73920700 \\ \mathrm{H} & -4.14674800 & -7.32894800 & 0.87579600 \\ \mathrm{C} & -0.75329800 & -7.46932800 & 2.75177000 \\ \mathrm{H} & 0.12741100 & -6.82177000 & 0.88289700 \\ \mathrm{C} & -1.92696500 & -7.81116900 & 3.43454200 \\ \mathrm{H} & -4.06856800 & -8.01957300 & 3.25260400 \\ \mathrm{H} & 0.20001100 & -7.51237200 & 3.27154200 \\ \mathrm{C} & -1.89351200 & -8.23734200 & 4.88196200 \\ \mathrm{H} & -2.29704300 & -9.24968900 & 5.00403500 \\ \mathrm{H} & -0.87484200 & -8.23126800 & 5.27995700 \\ \mathrm{H} & -2.50344300 & -7.57144100 & 5.50428600\end{array}$

TS4

C

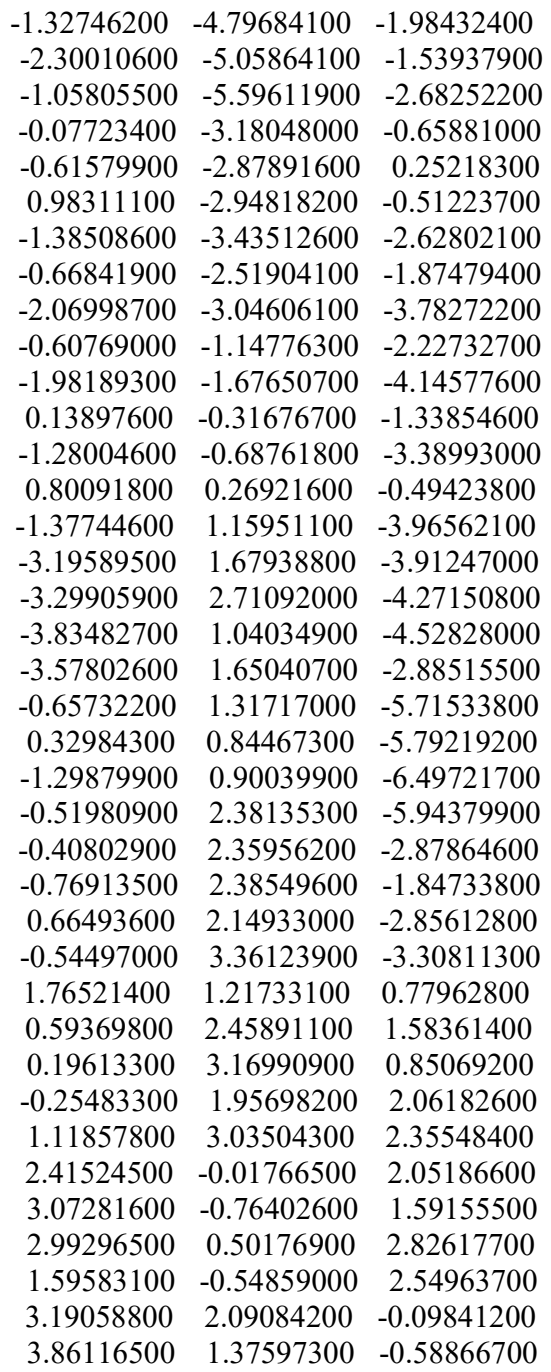

$\begin{array}{lll}2.82537800 & 2.78897400 & -0.85996100\end{array}$ $\begin{array}{lll}3.78618300 & 2.66597200 & 0.62108900\end{array}$ $-3.57403300 \quad-4.34032000 \quad-4.55672200$ $\begin{array}{lll}-0.25653500 & -4.62110200 & -0.96914100\end{array}$ $\begin{array}{lll}-4.31712000 & -0.58211000 & -7.59980500\end{array}$ $\begin{array}{lll}-3.12661200 & -1.32767100 & -7.69865900\end{array}$ $\begin{array}{lll}-2.65819500 & -1.72935600 & -8.96155000\end{array}$ $\begin{array}{llll}-3.36813200 & -1.38464100 & -10.10923900\end{array}$ $\begin{array}{lll}-4.54806800 & -0.64435900 & -10.00578600\end{array}$ $\begin{array}{lll}-5.02031400 & -0.24446600 & -8.74983600\end{array}$ $\begin{array}{lll}-4.67280800 & -0.27562100 & -6.62194400\end{array}$ $\begin{array}{lll}-1.73918700 & -2.30094900 & -9.04304000\end{array}$ $\begin{array}{llll}-3.00052200 & -1.69260000 & -11.08311900\end{array}$ $\begin{array}{lll}-5.10086600 & -0.37827900 & -10.90189500\end{array}$ $\begin{array}{lll}-5.93703000 & 0.33176600 & -8.67109200\end{array}$ $\begin{array}{lll}-2.39411000 & -1.66999000 & -6.48004700\end{array}$ $\begin{array}{lll}-2.65980800 & -1.27622000 & -5.30292800\end{array}$ $\begin{array}{llll}0.45234300 & -4.09232800 & -5.55332300\end{array}$ $\begin{array}{llll}1.44364600 & -3.66880500 & -4.64162800\end{array}$ $\begin{array}{lll}2.47301200 & -4.54219200 & -4.31297000\end{array}$ $\begin{array}{llll}2.51031200 & -5.82590700 & -4.86862300\end{array}$ $\begin{array}{lll}1.51973100 & -6.24823100 & -5.76399000\end{array}$ $\begin{array}{lll}0.48547700 & -5.39017900 & -6.11366100\end{array}$ $\begin{array}{lll}1.39431600 & -2.67396800 & -4.21346500\end{array}$ $\begin{array}{lll}3.24474000 & -4.22568400 & -3.61906300\end{array}$ $\begin{array}{llll}3.31637700 & -6.50330800 & -4.60335000\end{array}$ $\begin{array}{llll}1.55929600 & -7.24502100 & -6.19131300\end{array}$ $\begin{array}{lll}-0.28354500 & -5.70054000 & -6.81345700\end{array}$ $\begin{array}{lll}-0.57483300 & -3.19991900 & -5.93850200\end{array}$ $\begin{array}{lll}-1.22537300 & -2.52404000 & -6.67610000\end{array}$ $\begin{array}{lll}-0.37351000 & -5.68544500 & 0.35741300\end{array}$ $\begin{array}{lll}-0.74978100 & -6.97004800 & -0.24079100\end{array}$ $\begin{array}{lll}-1.19121800 & -5.08197300 & 1.41813900\end{array}$ $\begin{array}{lll}1.31576200 & -5.75932500 & 0.93381200\end{array}$ $\begin{array}{lll}1.68206300 & -5.05898300 & 2.08348100\end{array}$ $\begin{array}{lll}2.23869800 & -6.55508300 & 0.24786100\end{array}$ $\begin{array}{lll}2.99821000 & -5.14685600 & 2.53856400\end{array}$ $\begin{array}{llll}0.94289500 & -4.47217300 & 2.61814700\end{array}$ $\begin{array}{lll}3.54545100 & -6.63135100 & 0.71879500\end{array}$ $\begin{array}{llll}1.92663600 & -7.11654200 & -0.62655000\end{array}$ $\begin{array}{llll}3.94698100 & -5.93121000 & 1.86942900\end{array}$ $\begin{array}{lll}3.28796300 & -4.60613600 & 3.43563800\end{array}$ $\begin{array}{llll}4.26647200 & -7.25447900 & 0.19524300\end{array}$ $\begin{array}{lll}5.35941000 & -6.05369000 & 2.38801000\end{array}$ $\begin{array}{lll}6.08902600 & -6.05051200 & 1.57091500\end{array}$ $\begin{array}{lll}5.60893300 & -5.23756500 & 3.07258800\end{array}$ $\begin{array}{lll}5.49247300 & -6.99534500 & 2.93610500\end{array}$

$\begin{array}{lccc}\text { IN7 } & & & \\ \text { C } & -2.69485600 & -4.25203700 & -0.20457100 \\ \text { H } & -3.43653700 & -4.46183300 & 0.58004100 \\ \text { H } & -2.77074400 & -5.02161500 & -0.97848300 \\ \text { C } & -0.85678600 & -2.81294600 & 0.52130700 \\ \text { H } & -0.91610900 & -2.48991400 & 1.57015700 \\ \text { H } & 0.18039200 & -2.71181400 & 0.18931000 \\ \text { C } & -2.87626300 & -2.85980100 & -0.73673100 \\ \text { C } & -1.83726700 & -2.05999900 & -0.34463800 \\ \text { C } & -3.92129400 & -2.34711700 & -1.53923400 \\ \text { C } & -1.76617600 & -0.68717000 & -0.72749200 \\ \text { C } & -3.81985600 & -0.98906900 & -2.00216200 \\ \text { C } & -0.65022700 & 0.03330400 & -0.19907900 \\ \text { C } & -2.74398900 & -0.12779500 & -1.57266300 \\ \text { C } & 0.33493100 & 0.54311600 & 0.31524700 \\ \text { Si } & -2.57634100 & 1.74407700 & -2.05655400 \\ \text { C } & -2.67598400 & 2.73486800 & -0.44424000 \\ \text { H } & -2.48941900 & 3.79558800 & -0.65480000 \\ \text { H } & -3.68034200 & 2.66244600 & -0.00924500\end{array}$


$\begin{array}{lll}-1.74400300 & -5.56338800 & -1.79466000\end{array}$ $\begin{array}{lll}-2.80072900 & -5.86467800 & -1.77630000\end{array}$ $\begin{array}{lll}-1.18902600 & -6.35359700 & -2.30406600\end{array}$ $\begin{array}{lll}-1.28735000 & -3.95304500 & -0.04904100\end{array}$ $\begin{array}{lll}-2.16875300 & -3.73490900 & 0.56799500\end{array}$ $\begin{array}{lll}-0.40427100 & -3.66947600 & 0.52929800\end{array}$ $\begin{array}{lll}-1.57780900 & -4.18749400 & -2.40514100\end{array}$ $\begin{array}{lll}-1.35313000 & -3.26352100 & -1.38947700\end{array}$ $\begin{array}{lll}-1.65053100 & -3.82820900 & -3.75227700\end{array}$ $\begin{array}{lll}-1.26305500 & -1.88365500 & -1.65397200\end{array}$ $\begin{array}{lll}-1.50515300 & -2.43543900 & -3.99865500\end{array}$ $\begin{array}{lll}-1.06338700 & -1.03826200 & -0.51763300\end{array}$ $\begin{array}{lll}-1.37716600 & -1.42732400 & -2.99703100\end{array}$ $\begin{array}{lll}-0.88152600 & -0.44471700 & 0.53544400\end{array}$ $\begin{array}{llll}-1.42940600 & 0.42693300 & -3.52594400\end{array}$ $\begin{array}{lll}-3.11789100 & 0.75237200 & -4.32308000\end{array}$ $\begin{array}{lll}-3.15447000 & 1.77244400 & -4.72597100\end{array}$ $\begin{array}{lll}-3.34122100 & 0.05459000 & -5.13557900\end{array}$ $\begin{array}{lll}-3.91653900 & 0.66576300 & -3.57649200\end{array}$ $\begin{array}{lll}-0.01013700 & 0.81460700 & -4.73146000\end{array}$ $\begin{array}{lll}0.95266000 & 0.45738400 & -4.34419000\end{array}$ $\begin{array}{llll}-0.15590100 & 0.39574600 & -5.73169200\end{array}$ $\begin{array}{llll}0.07543100 & 1.90255300 & -4.84509100\end{array}$ $\begin{array}{lll}-1.24653700 & 1.65960000 & -2.10484000\end{array}$ $\begin{array}{llll}-2.03200000 & 1.56045400 & -1.35083300\end{array}$ $\begin{array}{lll}-0.28131500 & 1.58567700 & -1.59571300\end{array}$ $\begin{array}{lll}-1.32204400 & 2.66531500 & -2.54054800\end{array}$ $\begin{array}{lll}-0.59598400 & 0.52732400 & 2.08228000\end{array}$ $\begin{array}{lll}-0.47438800 & -0.68067300 & 3.52954600\end{array}$ $\begin{array}{lll}-1.39031400 & -1.27298100 & 3.63705100\end{array}$ $\begin{array}{lll}0.36296500 & -1.37568300 & 3.40170700\end{array}$ $\begin{array}{lll}-0.31609100 & -0.13922100 & 4.47021900\end{array}$ $\begin{array}{lll}1.01874000 & 1.48887900 & 1.88286500\end{array}$ $\begin{array}{lll}0.97196600 & 2.17057400 & 1.02592600\end{array}$ $\begin{array}{lll}1.22387300 & 2.09133600 & 2.77636100\end{array}$ $\begin{array}{lll}1.87050700 & 0.81613900 & 1.73055600\end{array}$ $\begin{array}{lll}-2.04842900 & 1.71133000 & 2.31758700\end{array}$ $\begin{array}{lll}-2.14995300 & 2.38972200 & 1.46254000\end{array}$ $\begin{array}{lll}-2.99522400 & 1.17188100 & 2.43318200\end{array}$ $\begin{array}{lll}-1.90220900 & 2.32546400 & 3.21477300\end{array}$ $\begin{array}{lll}-2.21738600 & -5.26606600 & -5.18501400\end{array}$ $\begin{array}{llll}-1.26944900 & -5.40439700 & -0.39045400\end{array}$ $\begin{array}{lll}-1.97029800 & -1.13799800 & -8.08679400\end{array}$ $\begin{array}{lll}-0.89104700 & -1.94737000 & -7.68806400\end{array}$ $\begin{array}{lll}0.04392700 & -2.37610000 & -8.64369200\end{array}$ $\begin{array}{lll}-0.09410500 & -1.99618800 & -9.97693200\end{array}$ $\begin{array}{lll}-1.16323600 & -1.18743300 & -10.36678300\end{array}$ $\begin{array}{lll}-2.10002200 & -0.76112800 & -9.41894700\end{array}$ $\begin{array}{lll}-2.69244200 & -0.81499100 & -7.34503600\end{array}$ $\begin{array}{lll}0.87559500 & -3.00960500 & -8.35145100\end{array}$ $\begin{array}{llll}0.63317800 & -2.33137600 & -10.70987800\end{array}$ $\begin{array}{lll}-1.26856800 & -0.89061600 & -11.40609100\end{array}$ $\begin{array}{lll}-2.93334500 & -0.13370500 & -9.72064700\end{array}$ $\begin{array}{lll}-0.77230200 & -2.32078300 & -6.27252900\end{array}$ $\begin{array}{lll}-1.55732500 & -1.98835400 & -5.33704700\end{array}$ $\begin{array}{llll}2.44480500 & -3.84315200 & -4.56976200\end{array}$ $\begin{array}{lll}2.28718700 & -4.33215700 & -3.25248700\end{array}$ $\begin{array}{lll}3.40967000 & -4.77518700 & -2.56510300\end{array}$ $\begin{array}{lll}4.66820200 & -4.72437500 & -3.17338900\end{array}$ $\begin{array}{llll}4.82330700 & -4.22985500 & -4.47702200\end{array}$ $\begin{array}{lll}3.71872800 & -3.78443600 & -5.18710200\end{array}$ $\begin{array}{llll}1.31014900 & -4.35626100 & -2.78484300\end{array}$ $\begin{array}{lll}3.28080200 & -5.15566400 & -1.55820600\end{array}$ $\begin{array}{llll}5.54178800 & -5.06964200 & -2.62804000\end{array}$ $\begin{array}{lll}5.80679800 & -4.19354400 & -4.93471900\end{array}$ 


$\begin{array}{lrrr}\mathrm{H} & 3.82099000 & -3.39888600 & -6.19610200 \\ \mathrm{C} & 1.31890600 & -3.40661700 & -5.28352400 \\ \mathrm{~N} & 0.40973200 & -3.10417100 & -5.95023900 \\ \mathrm{~S} & 0.17480400 & -6.22649800 & -0.02642300 \\ \mathrm{O} & 1.35452400 & -5.35954800 & -0.23837500 \\ \mathrm{O} & 0.09593600 & -7.50343600 & -0.74422200 \\ \mathrm{C} & 0.01417900 & -6.48967900 & 1.73100800 \\ \mathrm{C} & -1.04007000 & -7.27556000 & 2.21030200 \\ \mathrm{C} & 0.96730700 & -5.95740000 & 2.59606700 \\ \mathrm{C} & -1.13626600 & -7.51281600 & 3.57630600 \\ \mathrm{H} & -1.77371300 & -7.68520300 & 1.52380800 \\ \mathrm{C} & 0.85326300 & -6.20668000 & 3.96479500 \\ \mathrm{H} & 1.78039700 & -5.35875700 & 2.20196300 \\ \mathrm{C} & -0.19203800 & -6.98500500 & 4.47606000 \\ \mathrm{H} & -1.95702200 & -8.11651300 & 3.95611500 \\ \mathrm{H} & 1.59103400 & -5.78960900 & 4.64473100 \\ \mathrm{C} & -0.30507900 & -7.27671500 & 5.95323400 \\ \mathrm{H} & -1.32353200 & -7.10123400 & 6.31713700 \\ \mathrm{H} & -0.06727200 & -8.32867400 & 6.16199100 \\ \mathrm{H} & 0.37999200 & -6.65893100 & 6.53999500 \\ \mathrm{C} & -3.91830800 & -8.64469900 & -8.04844600 \\ \mathrm{C} & -3.63722900 & -9.99402200 & -7.75763800 \\ \mathrm{C} & -4.18361500 & -10.98967000 & -8.56240800 \\ \mathrm{C} & -5.00263700 & -10.64915100 & -9.64338800 \\ \mathrm{C} & -5.28110400 & -9.30918600 & -9.92866900 \\ \mathrm{C} & -4.74297600 & -8.29963400 & -9.13682800 \\ \mathrm{H} & -3.00175100 & -10.24674600 & -6.91424400 \\ \mathrm{H} & -3.97220300 & -12.03204600 & -8.34513000 \\ \mathrm{H} & -5.42775300 & -11.43128600 & -10.26488400 \\ \mathrm{H} & -5.91739800 & -9.05017000 & -10.76858400 \\ \mathrm{H} & -4.95356300 & -7.25628000 & -9.34806600 \\ \mathrm{C} & -3.36623000 & -7.61333700 & -7.22889500 \\ \mathrm{~N} & -2.92715600 & -6.76952800 & -6.56317000\end{array}$

TS5

C

$\mathrm{H}$

$\mathrm{H}$

C

$\mathrm{H}$

$\mathrm{H}$

C

C

C $\begin{array}{lll}-3.58548300 & -4.95996900 & -2.12669400\end{array}$

$\begin{array}{lll}-4.63217400 & -4.76262100 & -1.85653700\end{array}$

$\begin{array}{lll}-3.57214400 & -5.83518000 & -2.77803300\end{array}$

$\begin{array}{lll}-2.25499300 & -3.91586700 & -0.42683700\end{array}$

$\begin{array}{lll}-2.92894800 & -3.47665100 & 0.31980200\end{array}$

$\begin{array}{lll}-1.28004000 & -4.04740100 & 0.04901300\end{array}$

$\begin{array}{lll}-2.92370600 & -3.72727000 & -2.70325900\end{array}$

$\begin{array}{lll}-2.19432200 & -3.09819600 & -1.69648300\end{array}$

$\begin{array}{lll}-2.95595000 & -3.22183600 & -3.99407100\end{array}$

$\begin{array}{lll}-1.56579900 & -1.86065200 & -1.91220700\end{array}$

$-2.21483200 \quad-2.04432900-4.23966500$

$\begin{array}{lll}-0.90819900 & -1.28879700 & -0.77831500\end{array}$

$\begin{array}{lll}-1.59878200 & -1.27714600 & -3.21273300\end{array}$

$\begin{array}{lll}-0.37674000 & -0.92746300 & 0.26149100\end{array}$

$\begin{array}{llll}-1.02230300 & 0.51687400 & -3.62886900\end{array}$

$\begin{array}{llll}-2.53741400 & 1.43997200 & -4.29446800\end{array}$

$\begin{array}{llll}-2.25543500 & 2.45292400 & -4.60815700\end{array}$

$\begin{array}{llll}-2.99336400 & 0.93099400 & -5.14815500\end{array}$

$\begin{array}{llll}-3.30107700 & 1.53968400 & -3.51375100\end{array}$

$\begin{array}{llll}0.39373600 & 0.53829200 & -4.89275200\end{array}$

$\begin{array}{lll}1.23667600 & -0.07653400 & -4.55605000\end{array}$

$\begin{array}{llll}0.09254100 & 0.19764600 & -5.88804400\end{array}$

$\begin{array}{lll}0.76273700 & 1.56682500 & -4.99503100\end{array}$

$\begin{array}{lll}-0.40539000 & 1.49382800 & -2.13243100\end{array}$

$\begin{array}{lll}-1.14126600 & 1.54349900 & -1.32495000\end{array}$

$\begin{array}{llll}0.52406600 & 1.09703700 & -1.71514000\end{array}$

$\begin{array}{lll}-0.21206900 & 2.51996400 & -2.47373400\end{array}$

$\begin{array}{lll}0.42866400 & -0.32167500 & 1.81126100\end{array}$

$\begin{array}{lll}0.54310600 & -1.76979900 & 3.02157900\end{array}$

$\begin{array}{lll}-0.44913900 & -2.16264000 & 3.27169800\end{array}$

$\begin{array}{lll}1.13720800 & -2.59328800 & 2.60869900\end{array}$

$\begin{array}{lll}1.01969700 & -1.45207200 & 3.95715800\end{array}$ $\begin{array}{llll}2.15580900 & 0.31293200 & 1.38011900\end{array}$

$\begin{array}{lll}2.11060500 & 1.13602500 & 0.65788600\end{array}$

$\begin{array}{lll}2.66482800 & 0.68571200 & 2.27754800\end{array}$

$\begin{array}{lll}2.77838500 & -0.47934200 & 0.94874600\end{array}$

$\begin{array}{lll}-0.62965200 & 1.06345600 & 2.53810100\end{array}$

$\begin{array}{lll}-0.72742600 & 1.90231100 & 1.83982200\end{array}$

$\begin{array}{lll}-1.63828700 & 0.71079800 & 2.78153900\end{array}$

$\begin{array}{lll}-0.17854600 & 1.44843000 & 3.46103500\end{array}$

$\begin{array}{lll}-4.21333800 & -4.05142400 & -5.47966300\end{array}$

$\begin{array}{lll}-2.82971800 & -5.22430600 & -0.86857200\end{array}$

$\begin{array}{lll}-1.95222400 & -0.51458000 & -8.24101700\end{array}$

$\begin{array}{lll}-1.36785100 & -1.74147000 & -7.88009100\end{array}$

$\begin{array}{lll}-0.69747600 & -2.49930100 & -8.85337500\end{array}$

$\begin{array}{llll}-0.60905700 & -2.03644800 & -10.16551100\end{array}$

$\begin{array}{llll}-1.18816600 & -0.81569300 & -10.51686700\end{array}$

$\begin{array}{lll}-1.85975500 & -0.05774800 & -9.55172400\end{array}$

$\begin{array}{llll}-2.46986600 & 0.06557700 & -7.48537500\end{array}$

$\begin{array}{lll}-0.25154600 & -3.45305200 & -8.58788900\end{array}$

$\begin{array}{llll}-0.08909800 & -2.62910400 & -10.91230100\end{array}$

$\begin{array}{llll}-1.11830600 & -0.45536400 & -11.53907300\end{array}$

$\begin{array}{lll}-2.31164600 & 0.89167400 & -9.82293800\end{array}$

$\begin{array}{lll}-1.46466900 & -2.20100500 & -6.48001400\end{array}$

$-2.09212000 \quad-1.57128100 \quad-5.56862500$

$\begin{array}{lll}1.27321200 & -3.76594300 & -4.83125200\end{array}$

$\begin{array}{lll}1.62603100 & -4.48311300 & -3.67518000\end{array}$

$\begin{array}{lll}2.82968900 & -4.19000400 & -3.04156500\end{array}$

$\begin{array}{llll}3.68475600 & -3.21407200 & -3.56270000\end{array}$

$\begin{array}{lll}3.33377500 & -2.50693400 & -4.71749600\end{array}$

$\begin{array}{lll}2.12544800 & -2.77110500 & -5.35278100\end{array}$

$\begin{array}{lll}0.95431000 & -5.22537100 & -3.25705600\end{array}$

$3.10001700 \quad-4.72725100 \quad-2.13821700$

$\begin{array}{lll}4.62898700 & -3.00358400 & -3.06901000\end{array}$

$\begin{array}{lll}4.00197400 & -1.75416300 & -5.12373300\end{array}$

$\begin{array}{llll}1.84240400 & -2.22925500 & -6.24947000\end{array}$

$\begin{array}{llll}0.01667200 & -3.96839800 & -5.51362600\end{array}$

$\begin{array}{lll}-0.81395500 & -3.44473100 & -6.20842300\end{array}$

$\begin{array}{lll}-1.68627000 & -6.48137900 & -0.99883800\end{array}$

$\begin{array}{lll}-0.37108800 & -6.01419600 & -1.48249800\end{array}$

$\begin{array}{lll}-2.36152900 & -7.54791600 & -1.76203200\end{array}$

$\begin{array}{lll}-1.50323600 & -6.97071900 & 0.70623700\end{array}$

$\begin{array}{lll}-2.62946100 & -7.36285400 & 1.43683300\end{array}$

$\begin{array}{lll}-0.22614500 & -7.01612700 & 1.26652800\end{array}$

$\begin{array}{lll}-2.46341500 & -7.79436800 & 2.74878800\end{array}$

$\begin{array}{lll}-3.61556100 & -7.32407600 & 0.98660300\end{array}$

$\begin{array}{lll}-0.08200900 & -7.45281000 & 2.58297000\end{array}$

$\begin{array}{lll}0.63333700 & -6.71441400 & 0.67830900\end{array}$

$\begin{array}{lll}-1.19175900 & -7.84486700 & 3.34417500\end{array}$

$\begin{array}{lll}-3.33464900 & -8.09906400 & 3.32263500\end{array}$

$\begin{array}{rrr}0.91019300 & -7.49046100 & 3.02491200\end{array}$

$\begin{array}{lll}-1.02985700 & -8.29371800 & 4.77602000\end{array}$

$\begin{array}{lll}-1.17007200 & -7.45287800 & 5.46748200\end{array}$

$\begin{array}{lll}-1.76651300 & -9.05909300 & 5.04095800\end{array}$

$\begin{array}{lll}-0.03144000 & -8.70386500 & 4.95730100\end{array}$

$\begin{array}{lll}-1.47126400 & -8.00640700 & -5.81151600\end{array}$

$\begin{array}{lll}-2.07637400 & -8.73684000 & -4.76859200\end{array}$

$\begin{array}{lll}-2.64542100 & -9.97204900 & -5.06657000\end{array}$

$\begin{array}{lll}-2.61232100 & -10.47142400 & -6.37191000\end{array}$

$\begin{array}{lll}-2.00752400 & -9.74050300 & -7.40125000\end{array}$

$\begin{array}{lll}-1.43218100 & -8.50483600 & -7.13094300\end{array}$

$\begin{array}{lll}-2.09288800 & -8.34364500 & -3.75580300\end{array}$

$\begin{array}{lll}-3.11297800 & -10.54686900 & -4.27350800\end{array}$

$\begin{array}{lll}-3.05772700 & -11.43751600 & -6.59057700\end{array}$

$\begin{array}{llll}-1.98311000 & -10.13651700 & -8.41146800\end{array}$

$\begin{array}{lll}-0.95531200 & -7.92960200 & -7.91804700\end{array}$

$\begin{array}{lll}-0.87415500 & -6.74625700 & -5.53431900\end{array}$

$\begin{array}{lll}-0.35016800 & -5.73311100 & -5.30938400\end{array}$ 

$\begin{array}{lll}-2.04263300 & -10.10266700 & -4.44497500\end{array}$ $\begin{array}{lll}-1.45016900 & -10.88177800 & -5.44357600\end{array}$ $\begin{array}{llll}-0.68798400 & -10.29075300 & -6.46032100\end{array}$ $\begin{array}{lll}-0.51418000 & -8.91467900 & -6.48655600\end{array}$ $\begin{array}{lll}-2.33657500 & -8.11430300 & -3.66125600\end{array}$ $\begin{array}{lll}-2.62677300 & -10.56928700 & -3.65855000\end{array}$ $\begin{array}{lll}-1.57990400 & -11.95997300 & -5.43178800\end{array}$ $\begin{array}{lll}-0.23123800 & -10.90533500 & -7.22918500\end{array}$ $\begin{array}{lll}0.07305200 & -8.44118100 & -7.26650000\end{array}$ $\begin{array}{llll}-0.94724500 & -6.73520400 & -5.51527600\end{array}$ $\begin{array}{llll}-0.77730700 & -5.59262700 & -5.61426000\end{array}$ 